\title{
DUALITY BY REPRODUCING KERNELS
}

\section{A. SHLAPUNOV and N. TARKHANOV}

\author{
Received 10 June 2002
}

\begin{abstract}
Let $A$ be a determined or overdetermined elliptic differential operator on a smooth compact manifold $X$. Write $\mathscr{S}_{A}(\mathscr{D})$ for the space of solutions of the system $A u=0$ in a domain $\mathscr{D} \Subset X$. Using reproducing kernels related to various Hilbert structures on subspaces of $\mathscr{Y}_{A}(\mathscr{D})$, we show explicit identifications of the dual spaces. To prove the regularity of reproducing kernels up to the boundary of $\mathscr{D}$, we specify them as resolution operators of abstract Neumann problems. The matter thus reduces to a regularity theorem for the Neumann problem, a well-known example being the $\bar{\partial}$-Neumann problem. The duality itself takes place only for those domains $\mathscr{D}$ which possess certain convexity properties with respect to $A$.
\end{abstract}

2000 Mathematics Subject Classification: 32C37, 58J10, 35N15, 46A20, $46 E 22$.

1. Introduction. The present work continues our paper [12] and grows out of a desire to highlight the link of duality theorems for solutions of elliptic equations and basic problems of partial differential equations such as the existence and regularity of solutions.

Let

$$
V^{0} \stackrel{T}{\longrightarrow} V^{1}, \quad V^{0} \stackrel{S}{\longleftarrow} V^{1}
$$

be two continuous mappings of Fréchet spaces such that $S T=I$ on a closed subspace $U^{0}$ of $V^{0}$. In other words, the identity mapping of $U^{0}$ factors through $T$, hence the restriction of $T$ to $U^{0}$ is one-to-one and the image of $U^{0}$ under $T$ is a closed subspace of $V^{1}$.

Obviously, $S$ maps $T U^{0}$ to $U^{0}$. If $\mathscr{F}$ is a continuous linear functional on $U^{0}$, then

$$
\langle\mathscr{F}, u\rangle=\left\langle S^{\prime} \mathscr{F}, T u\right\rangle
$$

for any $u \in U^{0}$, where $S^{\prime}$ is the transpose of $S: T U^{0} \rightarrow U^{0}$. Moreover, $S^{\prime} \mathscr{F}=0$ implies $\mathscr{F}=0$. We thus obtain a one-to-one mapping $\left(U^{0}\right)^{\prime} \rightarrow\left(T U^{0}\right)^{\prime}$ given by $\mathscr{F} \mapsto S^{\prime} \mathscr{F}$. The problem of identifying the dual of $U^{0}$ reduces to the description of the range of $S^{\prime}$.

We restrict our attention to the case where both $V^{0}$ and $V^{1}$ are function spaces and $U^{0}$ is a space of solutions of some elliptic equation $A u=0$. Even for explicitly given $S$, the range of $S^{\prime}$ cannot be described by mere tools of partial 
differential equations. The crucial fact is that by the uniqueness theorem, $C^{\infty}$ functions with compact support are not dense in $U^{0}$, and so the functionals of $\left(U^{0}\right)^{\prime}$ cannot be specified within distributions. To handle $S^{\prime}$, we therefore need much more refined analysis. For a deeper discussion, we refer the reader to [6] and [23, Chapter 3].

It is usually the case for hypoelliptic equations that $U^{0}$ is in fact a nuclear space. By the Schwartz kernel theorem, the mapping $S: T U^{0} \rightarrow U^{0}$ has a kernel $K_{S} \in U^{0} \hat{\otimes}_{\pi}\left(T U^{0}\right)^{\prime}$ (cf. [22, Section 1.4.1]). We call $K_{S}$ a reproducing kernel for

$$
u(x)=\left\langle K_{S}(x, \cdot), T u\right\rangle
$$

for all $x$ in the domain of $u \in U^{0}$.

The advantage of using reproducing kernels lies in the fact that it enables us to write $S^{\prime} \mathscr{F}=\left\langle\mathscr{F}, K_{S}(\cdot, y)\right\rangle$. The right-hand side here is called the indicatrix of the functional $\mathscr{F}$.

This concept was first studied in the particular cases of holomorphic and harmonic functions on certain explicitly given domains (cf. [2, 8, 25]). However, representing analytic functionals as analytic functions on domains in $\mathbb{C}^{n}$ requires specific tools of the general theory of partial differential equations (cf. [3, 4, 19, 26] and [20, Theorem 3.4]).

Our main result consists of the following. The correspondence $\mathscr{F} \mapsto S^{\prime} \mathscr{F}$ maps the dual space of $U^{0}$ to the space of the solutions of $A v=0$ in the same domain, which grow near the boundary in a sense dual to the growth of the solutions in $U^{0}$. This mapping is always one-to-one but not necessarily onto. Its surjectivity is equivalent to the regularity of certain projection onto the space of solutions.

We evaluate the projection through the resolution operator of a generalised Neumann problem related to $A$. The desired regularity of the projection just amounts to that of the solution of the Neumann problem. We thus bring together two different areas of analysis in which the problem of regularity turns out to be of key importance.

\section{A general scheme}

2.1. Spaces of the solutions of elliptic systems. Let $X$ be a $C^{\infty}$-manifold of dimension $n$ with a smooth boundary $\partial X$. The case $\partial X=\varnothing$ is also included. We tacitly assume that $X$ is embedded into a smooth closed manifold $\tilde{X}$ of the same dimension.

For any smooth $\mathbb{C}$-vector bundles $E$ and $F$ over $X$, we write $\operatorname{Diff}^{m}(X ; E, F)$ for the space of all linear partial differential operators of order $\leq m$ between sections of $E$ and $F$.

Denote by $E^{*}$ the conjugate bundle of $E$. Any Hermitean metric $(\cdot, \cdot)_{x}$ on $E$ gives rise to a sesquilinear bundle isomorphism $*_{E}: E \rightarrow E^{*}$ by the equality $\left\langle *_{E} v, u\right\rangle_{x}=(u, v)_{x}$ for all sections $u$ and $v$ of $E$. 
We pick a volume form $d x$ on $X$, thus identifying the dual and conjugate bundles. For $A \in \operatorname{Diff}^{m}(X ; E, F)$, denote by $A^{\prime} \in \operatorname{Diff}^{m}\left(X ; F^{*}, E^{*}\right)$ the transposed operator and by $A^{*} \in \operatorname{Diff}^{m}(X ; F, E)$ the formal adjoint operator. We obviously have

$$
A^{*}=*_{E}^{-1} A^{\prime} *_{F}
$$

(cf. [21, Section 4.1.4] and elsewhere).

Write $\sigma^{m}(A)$ for the principal homogeneous symbol of order $m$ of the operator $A, \sigma^{m}(A)$ living on the cotangent bundle $T^{*} X$ of $X$. From now on, we assume that $\sigma^{m}(A)$ is injective away from the zero section of $T^{*} X$. Hence, it follows that the Laplacian $\Delta=A^{*} A$ is an elliptic differential operator of order $2 m$ on $X$.

Given any open set $U$ in $\stackrel{\circ}{X}$, the interior of $X$, let $\mathscr{S}_{A}(U)$ stand for the space of the solutions of the equation $A u=0$ in $U$ with the topology of uniform convergence on compact subsets of $U$. It is known that $\mathscr{S}_{A}(U)$ is a FréchetSchwartz space.

Denote by $\mathscr{Y}_{A}(U)^{\prime}$ the dual space of $\mathscr{Y}_{A}(U)$, that is, the space of all continuous linear functionals on $\mathscr{Y}_{A}(U)$. As usual, we give $\mathscr{Y}_{A}(U)^{\prime}$ the strong topology, that is, the topology of uniform convergence of functionals on bounded subsets of $\mathscr{Y}_{A}(U)$.

Throughout this paper, we assume that the Laplacian $\Delta$ possesses the following unique continuation property:

$(U)_{s}$ given any domain $\mathscr{D} \subset \stackrel{\circ}{X}$, if $u \in \mathscr{S}_{\Delta}(\mathscr{D})$ vanishes on a nonempty open subset of $\mathscr{D}$, then $u \equiv 0$ in $\mathscr{D}$.

This property implies in particular the existence of a two-sided fundamental solution for $\Delta$ in the interior of $X$.

Natural domains for the solutions of $A u=0$ are certainly open subsets of the interior of $X$. However, some problems require to consider solutions on sets $\sigma$ in $X$ which are not open. Here, we are interested not simply in the restrictions of the solutions of the given set, but also in the local solutions of the system $A u=0$ on $\sigma$. By these, we mean solutions of the system on various neighbourhoods of $\sigma$ depending on the solution.

If $\sigma$ is a closed subset of $X$, then $\mathscr{Y}_{A}(\sigma)$ stands for the space of (equivalence classes of) local solutions of $A u=0$ on $\sigma$. Two such solutions are equivalent if there is a neighbourhood of $\sigma$ where they are equal. In $\mathscr{Y}_{A}(\sigma)$, a sequence $\left\{u_{v}\right\}$ is said to converge if there exists a neighbourhood $\mathcal{N}$ of $\sigma$ such that all the solutions are defined at least in $\mathcal{N}$ and converge uniformly on compact subsets of $\mathcal{N}$.

Alternatively, the space $\mathscr{S}_{A}(\sigma)$ can be described as the inductive limit of spaces $\mathscr{S}_{A}\left(U_{v}\right)$, where $\left\{U_{v}\right\}$ is any decreasing sequence of open sets containing $\sigma$, such that each neighbourhood of $\sigma$ contains some $U_{v}$ and such that each connected component of each $U_{v}$ intersects $\sigma$. This latter condition guarantees 
that the mappings $\mathscr{Y}_{A}\left(U_{v}\right) \rightarrow \mathscr{Y}_{A}(\sigma)$ are one-to-one. Then, $\mathscr{Y}_{A}(\sigma)$ is necessarily a Hausdorff space.

For an open set $U \subset X$, we denote by $L^{2}(U, E)$ the Hilbert space of all square integrable sections of $E$ over $U$ with scalar product

$$
(u, v)_{L^{2}(U, E)}=\int_{U}(u, v)_{x} d x .
$$

More generally, write $H^{s}(U, E), s \in \mathbb{Z}_{+}$, for the Sobolev space of the sections of $E$ over $U$, whose weak derivatives up to order $s$ belong to $L^{2}(U, E)$. We define $H^{s}(U, E)$ with $s=-1,-2, \ldots$ to be the dual space for $H^{-s}(U, E)$ with respect to the $L^{2}(U, E)$-pairing.

We also denote by $\mathscr{Y}_{A}^{(s)}(U)$, with any integer $s$, the closed subspace of $H^{s}(U, E)$ consisting of all weak solutions of $A u=0$ in $U$. It is well known that $\mathscr{S}_{A}^{(s)}(U)$ is a separable Hilbert space with reproducing kernel (cf. [22] and elsewhere).

The union of the spaces $\mathscr{Y}_{A}^{(s)}(U)$ over all $s \in \mathbb{Z}$ is perhaps of particular interest. For regular $U$, it consists of all the solutions of $A u=0$ in $U$, which are of finite order of growth near the boundary of $U$. This means that $u$ fulfills an estimate

$$
|u(x)| \leq \frac{C}{\operatorname{dist}(x, \partial U)^{N}}
$$

for all $x \in U$, with $N$ and $C$ constants depending on $u$.

Write $\mathscr{Y}_{A}^{(f)}(U)$ for the space of the solutions of $A u=0$ in $U$ which have a finite order of growth near $\partial U$. We give $\mathscr{S}_{A}^{(f)}(U)$ the inductive limit topology of the sequence $\mathscr{S}_{A}^{(-s)}(U), s \in \mathbb{N}$.

Since the Dirichlet problem for the Laplacian $\Delta=A^{*} A$ in $U$ is uniquely solvable, the topology of $\mathscr{S}_{\Delta}^{(f)}(U)$ can be equivalently described in the following way. Pick a Dirichlet system $u \mapsto t(u)$ of order $m-1$ on the boundary of $U$ provided the latter is smooth. By [18], for each $u \in \mathscr{Y}_{\Delta}^{(f)}(U)$, the Dirichlet data $t(u)$ are well defined in

$$
\bigoplus_{j=0}^{m-1} \mathscr{D}^{\prime}\left(\partial U, F_{j}\right),
$$

$F_{j}$ being some vector bundles in a neighbourhood of $\partial U$.

LEMMA 2.1. A sequence $\left\{u_{v}\right\}$ converges to $u$ in the space $\mathscr{Y}_{\Delta}^{(f)}(U)$ if and only if $t\left(u_{v}\right) \rightarrow t(u)$ in $\bigoplus_{j=0}^{m-1} \mathscr{D}^{\prime}\left(\partial U, F_{j}\right)$.

Proof. See Theorem 2.32.

2.2. Duality. Let $\Sigma_{1}$ be a vector subspace of $\mathscr{S}_{A}(U)$ endowed with topology $\tau_{1}$ which is not weaker than the Fréchet-Schwartz topology of $\mathscr{S}_{A}(U)$. Denote by $\Sigma_{1}^{\prime}$ the dual space of $\Sigma_{1}$. 
Suppose that $V$ is a separable Hilbert space of functions in a domain $U$ with a scalar product $h(\cdot, \cdot)$. Moreover, let there be a topological vector space $\Sigma_{2}$, continuous linear mappings

$$
\Sigma_{2} \stackrel{i_{2}}{\longrightarrow} V \stackrel{i_{1}}{\longrightarrow} \Sigma_{1}
$$

and a sesquilinear pairing

$$
\tilde{h}(\cdot, \cdot): \Sigma_{1} \times \Sigma_{2} \longrightarrow \mathbb{C}
$$

such that

(1) $\tilde{h}(\cdot, \cdot)$ is separately continuous;

(2) $\tilde{h}\left(i_{1} u, v\right)=h\left(u, i_{2} v\right)$ for all $u \in V$ and $v \in \Sigma_{2}$.

Under these assumptions, the pairing $\tilde{h}(\cdot, \cdot)$ induces a continuous mapping $\mathscr{g}: \Sigma_{2} \rightarrow \Sigma_{1}^{\prime}$ by

$$
(\mathscr{F} v)(\cdot):=\mathscr{F}_{v}(\cdot):=\tilde{h}(\cdot, v)
$$

for any $v \in \Sigma_{2}$.

LEMMA 2.2. If $i_{2}: \Sigma_{2} \rightarrow V$ is one-to-one, then the mapping g given by (2.7) is one-to-one.

Proof. If $\mathscr{F}_{v}(\cdot)$ is identically zero then

$$
\mathscr{F}_{v}\left(i_{1} i_{2} v\right)=h\left(i_{2} v, i_{2} v\right)=0
$$

As $h(\cdot, \cdot)$ is a scalar product on $V$, we conclude that $i_{2} v=0$. Hence $v=0$ if $i_{2}$ is one-to-one.

By a priori estimates for the solutions of elliptic systems, it is easy to see that the inclusion

$$
i=i_{1}: V \longrightarrow \mathscr{S}_{A}(U)=: \Sigma_{1}
$$

is continuous if and only if all the evaluation functionals $x \mapsto u(x), x \in U$, are continuous on $V$. This latter just amounts to saying that the Hilbert space $V$ has a reproducing kernel $K(x, y) \in V^{\prime} \otimes V$, that is, any $u \in V$ can be represented by the formula

$$
u(x)=h(u, K(x, \cdot))
$$

for all $x \in U$ (cf. [1]).

Define $*_{V}: V \rightarrow V^{\prime}$ by $\left\langle *_{V} v, u\right\rangle=h(u, v)$ for all $u \in V$. By the theorem of Riesz, $*_{V}$ is a sesquilinear isomorphism of $V$ onto $V^{\prime}$. 
THEOREM 2.3. Let $V$ be a Hilbert space with reproducing kernel $K(\cdot, \cdot)$. Suppose that $i_{2}$ is one-to-one. Then, the mapping given by (2.7) is onto if and only if

(1) $i_{1} i_{2}\left(\Sigma_{2}\right)$ is dense in $\Sigma_{1}$;

(2) for every $\mathscr{F}_{F} \in \Sigma_{1}^{\prime}$, the section $x \mapsto *_{V}^{-1}\left\langle\mathscr{F}, i_{1} K(x, \cdot)\right\rangle$ belongs to $i_{2}\left(\Sigma_{2}\right)$.

Note that

$$
h\left(u(y), *_{V} w(x) \otimes v(y)\right)=h(u, v) w(x)
$$

for each $x \in U$ and $u, v, w \in V$, as is easy to check.

\section{PROOF}

NeCESSITY. Let $\mathscr{F}_{F}$ be a continuous linear functional on $\Sigma_{1}$ vanishing on $i_{1} i_{2}\left(\Sigma_{2}\right)$. By the Hahn-Banach theorem, we prove that $i_{1} i_{2}\left(\Sigma_{2}\right)$ is dense in $\Sigma_{1}$ once we show that $\mathscr{F} \equiv 0$.

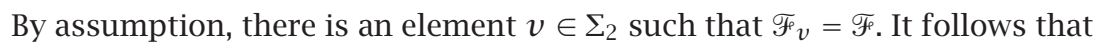

$$
\mathscr{F}_{v}\left(i_{1} i_{2} v\right)=h\left(i_{2} v, i_{2} v\right)=0,
$$

and so $v=0$. Hence $\mathscr{F} \equiv 0$ as desired.

Further, an easy calculation shows that

$$
\begin{aligned}
\left\langle\mathscr{F}, i_{1} K(x, \cdot)\right\rangle & =\tilde{h}\left(i_{1} K(x, \cdot), v\right) \\
& =h\left(K(x, \cdot), i_{2} v\right) \\
& =*_{V} h\left(i_{2} v, K(x, \cdot)\right) \\
& =*_{V}\left(i_{2} v\right)(x) \\
& \in *_{V} i_{2}\left(\Sigma_{2}\right),
\end{aligned}
$$

the fourth equality being due to the fact that $K(\cdot, \cdot)$ is a reproducing kernel of $V$. This proves the necessity.

SuFficiency. Let conditions (1) and (2) of the theorem hold. The task is now to show that the mapping $\mathscr{f}: \Sigma_{2} \rightarrow \Sigma_{1}^{\prime}$ is onto.

LEMMA 2.4. Let $u \in \Sigma_{1}$. Then, the formula

$$
u(x)=\tilde{h}\left(u, i_{2}^{-1} i_{1} K(x, \cdot)\right)
$$

is valid for all $x \in U$.

Proof. Indeed, by a priori estimates for elliptic systems all evaluation functionals $\delta_{x}(u)=u(x), x \in U$, are continuous on $\Sigma_{1}$. The condition (2) then implies that

$$
*_{V}^{-1}\left\langle\delta_{x}, i_{1} K(y, \cdot)\right\rangle=i_{1} *_{V}^{-1} K(y, x)=i_{1} K(x, y) \in E_{x}^{*} \otimes i_{2}\left(\Sigma_{2}\right)
$$

for every fixed $x \in U$. It follows that the pairing $\tilde{h}\left(u, i_{2}^{-1} i_{1} K(x, \cdot)\right)$ is well defined. 
Pick a sequence $\left\{u_{v}\right\}$ in $\Sigma_{2}$ such that $\left\{i_{1} i_{2} u_{v}\right\}$ approximates $u$ in $\Sigma_{1}$. Since $K(\cdot, \cdot)$ is a reproducing kernel, we see that

$$
\left(i_{2} u_{v}\right)(x)=h\left(i_{2} u_{v}, K(x, \cdot)\right)
$$

whence

$$
\left(i_{1} i_{2} u_{v}\right)(x)=h\left(i_{2} u_{v}, i_{2} i_{2}^{-1} i_{1, x} K(x, \cdot)\right)=\tilde{h}\left(i_{1} i_{2} u_{v}, i_{2}^{-1} i_{1, x} K(x, \cdot)\right)
$$

for all $x \in U$ and $v=1,2, \ldots$. Since the pairing $\tilde{h}(\cdot, \cdot)$ is separately continuous, the passage to the limit in (2.17), when $v \rightarrow \infty$, yields (2.14). The lemma is proved.

We are now in a position to complete the proof of Theorem 2.3. Suppose that $\mathscr{F} \in \Sigma_{1}^{\prime}$. Then by Lemma 2.4 , we see that

$$
\mathscr{F}(u)=\mathscr{F}\left(\tilde{h}\left(u, i_{2}^{-1} i_{1} K(x, \cdot)\right)\right)=\tilde{h}(u, v),
$$

where

$$
v=i_{2}^{-1}\left\langle\overline{\mathscr{F}}, i_{1} K(\cdot, y)\right\rangle=i_{2}^{-1} *_{V}^{-1}\left\langle\overline{\mathscr{F}}, i_{1} K(y, \cdot)\right\rangle \in \Sigma_{2}
$$

The last reasoning is an immediate consequence of condition (2), thus showing the theorem.

COROLLARY 2.5. If $\Sigma_{1}$ is a closed subspace of $\mathscr{Y}_{A}(U)$, then condition (2) of Theorem 2.3 is equivalent to the following one:

$\left(2^{\prime}\right)$ for each fixed $y \in U$, the section $i_{1, y} *_{V}^{-1} K(\cdot, y)$ belongs to $i_{2}\left(\Sigma_{2}\right) \otimes E_{y}$.

Proof. That (2) implies ( $\left.2^{\prime}\right)$ we have already established in the proof of Lemma 2.4. It remains to show the implication $\left(2^{\prime}\right) \Rightarrow(2)$.

Pick a continuous linear functional $\mathscr{F}_{F}$ on $\Sigma_{1}$. Since $\Sigma_{1}$ is a closed subspace of $C_{\text {loc }}(U, E)$, the space of continuous sections of $E$ over $U$, this functional extends, by the Hahn-Banach theorem, to an $E^{*}$-valued measure $m$ with a compact support in $U$. For any $x \in U$,

$$
*_{V}^{-1}\left\langle\mathscr{F}, i_{1} K(x, \cdot)\right\rangle=\int_{\operatorname{supp} m}\left\langle i_{1, y} *_{V}^{-1} K(x, y), d m(y)\right\rangle_{y} \in i_{2}\left(\Sigma_{2}\right)
$$

since $i_{1}$ is continuous and $\operatorname{supp} m$ is a compact subset of $U$. This completes the proof.

COROLLARY 2.6. Let $i_{2}$ be one-to-one. Suppose that the closed graph theorem is valid for mappings between $\Sigma_{2}$ and $\Sigma_{1}^{\prime}$. Then, the mapping $\mathscr{F}: \Sigma_{2} \rightarrow \Sigma_{1}^{\prime}$ defined by (2.7) is a topological isomorphism between these spaces if and only if conditions (1) and (2) of Theorem 2.3 hold.

Proof. This follows from the continuity of the mapping $\mathscr{f}$ and the closed graph theorem. 
Let $\Im_{1}, \Im_{2}$, and $\mathcal{V}$ be closed subspaces of the spaces $\Sigma_{1}, \Sigma_{2}$, and $V$, respectively. We thus get a commutative diagram

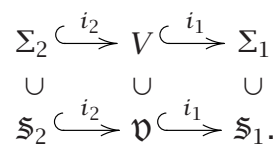

Once again, the pairing $\tilde{h}(\cdot, \cdot)$ induces the mapping $\mathfrak{J}: \Im_{2} \rightarrow \Im_{1}^{\prime}$ which is to a certain extent the restriction of $\mathscr{f}$ to $\$_{2}$. We tacitly assume that the continuous mappings under study are characterised in terms of convergent sequences and that the closed graph theorem is valid for mappings between $\Sigma_{2}$ and $\Sigma_{1}^{\prime}$.

Write $\pi: V \rightarrow \mathcal{V}$ for the corresponding orthogonal projection.

COROLLARY 2.7. Let $i_{2}$ be one-to-one. Suppose that $\&$ is a topological isomorphism of $\Sigma_{2}$ onto $\Sigma_{1}^{\prime}$. Then, the mapping $\mathfrak{J}$ is a topological isomorphism of $\mathfrak{\Im}_{2}$ onto $\Im_{1}^{\prime}$ if and only if

(1) $i_{1} i_{2} \Im_{2}$ is dense in $\Im_{1}$;

(2) the projection $\pi$ maps $i_{2}\left(\Sigma_{2}\right)$ continuously into $i_{2}\left(\$_{2}\right)$.

Proof. Since $i_{2}$ is one-to-one, so is the restriction of $i_{2}$ to $\Im_{2}$, too. Hence, the mapping $\mathfrak{J}$ is one-to-one by Lemma 2.2. It remains to prove that conditions (1) and (2) of Theorem 2.3, if applied to $\mathfrak{\Im}_{1}, \mathfrak{\Im}_{2}$, and $\mathfrak{V}$, are equivalent to conditions (1) and (2) of the present corollary. Of course, the conditions labelled with (1) coincide. Thus, we restrict our attention to the conditions labelled with (2).

NeCESSITY. Pick a sequence $\left\{v_{v}\right\}$ converging in $\Sigma_{2}$ to a limit $v$. Then, the corresponding sequence of functionals $\mathscr{F}_{v}$ converges to $\mathscr{F}_{v}$ in $\Sigma_{1}^{\prime}$. Clearly, the restrictions of $\mathscr{F}_{v_{v}}$ to $\Im_{1}$ converge in turn to the restriction of $\mathscr{F}_{v}$ to $\Im_{1}$ in the dual space $\mathfrak{\Im}_{1}^{\prime}$. If the mapping $\mathfrak{J}$ is a topological isomorphism of $\mathfrak{\Im}_{2}$ onto $\mathfrak{\Im}_{1}^{\prime}$, then there exists a sequence $\boldsymbol{v}_{v} \in \mathfrak{\Im}_{2}$ converging to a limit $\boldsymbol{v}$ in this space such that

$$
\mathscr{F}_{v_{v}}(\mathfrak{u})=\tilde{h}\left(\mathfrak{u}, \mathfrak{v}_{v}\right), \quad \mathscr{F}_{v}(\mathfrak{u})=\tilde{h}(\mathfrak{u}, \mathfrak{v})
$$

for all $\mathfrak{u} \in \mathfrak{\Im}_{1}$. In particular, for all $\mathfrak{u} \in \mathfrak{V}$, we get

$$
\begin{aligned}
h\left(\mathfrak{u}, \pi i_{2} v_{v}\right) & =h\left(\pi \mathfrak{u}, i_{2} v_{v}\right) \\
& =h\left(\mathfrak{u}, i_{2} v_{v}\right) \\
& =\tilde{h}\left(i_{1} \mathfrak{u}, v_{v}\right) \\
& =\mathscr{F} v_{v}\left(i_{1} \mathfrak{u}\right) \\
& =\tilde{h}\left(i_{1} \mathfrak{u}, \mathfrak{v}_{v}\right) \\
& =h\left(\mathfrak{u}, i_{2} \mathfrak{v}_{v}\right),
\end{aligned}
$$

that is, $\pi i_{2} v_{v}=i_{2} \mathcal{v}_{v}$ and the projection $\pi$ maps $i_{2}\left(\Sigma_{2}\right)$ continuously into $i_{2}\left(\Im_{2}\right)$ as desired. 
Sufficiency. Conversely, let conditions (1) and (2) of Corollary 2.7 hold. Pick an orthonormal basis $\left\{b_{v}\right\}$ in $\mathcal{V}$. It is well known that the reproducing kernel of $\mathfrak{V}$ is given in the form

$$
\mathfrak{K}(x, y)=\sum_{v} *_{\mathfrak{v}} b_{v}(x) \otimes b_{v}(y)
$$

As every orthonormal basis in $\mathfrak{D}$ can be extended to an orthonormal basis in $V$, we see that

$$
\mathfrak{K}(x, y)=\pi_{y} K(x, y)
$$

for all $x$ and $y$. By Theorem 2.3, the section $x \mapsto *_{V}^{-1}\left\langle\mathscr{F}, i_{1} K(x, \cdot)\right\rangle$ belongs to $i_{2}\left(\Sigma_{2}\right)$ for all $\mathscr{F}_{\mathfrak{F}} \in \Sigma_{1}^{\prime}$. By the Hahn-Banach theorem, every functional $\mathfrak{f} \in \mathfrak{S}_{1}^{\prime}$ actually extends continuously to a functional $\mathscr{F} \in \Sigma_{1}^{\prime}$. Hence condition (2) yields

$$
*_{V}^{-1}\left\langle\mathfrak{f}, i_{1} \mathfrak{K}(x, \cdot)\right\rangle=*_{V}^{-1}\left\langle\mathscr{F}_{F}, i_{1} \mathfrak{K}(x, \cdot)\right\rangle=\pi_{x} *_{V}^{-1}\left\langle\mathscr{F}_{F}, i_{1} K(x, \cdot)\right\rangle \in i_{2}\left(\Im_{2}\right),
$$

the latter inclusion being due to the commutative diagram (2.21). We thus conclude that condition (2) of Theorem 2.3 is fulfilled for $\mathfrak{J}$ and that this mapping is onto. The topological arguments now follow from the closed graph theorem.

Note that if $\operatorname{dim} \Sigma_{1}<\infty$, then conditions (1) and (2) of Theorem 2.3 imply that $i_{1} i_{2}\left(\Sigma_{2}\right)=\Sigma_{1}$ and $\operatorname{dim} \Sigma_{1}=\operatorname{dim} \Sigma_{2}$. Conversely, suppose that these latter conditions $i_{1} i_{2}\left(\Sigma_{2}\right)=\Sigma_{1}$ and $\operatorname{dim} \Sigma_{1}=\operatorname{dim} \Sigma_{2}$ are fulfilled. Hence, it follows that $i_{2}\left(\Sigma_{2}\right)$ is a closed subspace of $V$. Replacing $V$ by $i_{2}\left(\Sigma_{2}\right)$ and $i_{1}$ by its restriction to $i_{2}\left(\Sigma_{2}\right)$, we still have the same mapping $\mathscr{f}$. The reproducing kernel $K(\cdot, \cdot)$ is given by

$$
K(x, y)=\sum_{v=1}^{\operatorname{dim} \Sigma_{1}}\left(i_{2} b_{v}\right)^{*}(x) \otimes\left(i_{2} b_{v}\right)(y)
$$

where $\left\{b_{v}\right\}$ is a basis in $\Sigma_{2}$ with the property that $\left\{i_{2} b_{v}\right\}$ is an orthonormal basis in $i_{2}\left(\Sigma_{2}\right)$. Given any $\mathscr{F} \in \Sigma_{1}^{\prime}$, we get

$$
*_{V}^{-1}\left\langle\mathscr{F}, i_{1} K(x, \cdot)\right\rangle=\sum_{v=1}^{\operatorname{dim} \Sigma_{1}} \overline{\mathscr{F}}\left(i_{1} i_{2} b_{v}\right)\left(i_{2} b_{v}\right)(x) \in i_{2}\left(\Sigma_{2}\right) .
$$

Similar considerations apply to the commutative diagram (2.21). In this setting, the projection $\pi$ always maps $V$ continuously into $i_{2}\left(\$_{2}\right)$.

How can we derive the necessary information on the projection $\pi$ under general assumptions? In many cases, it can be obtained from a Neumann problem. 
2.3. Neumann problem. In our applications, $\mathfrak{D}$ is usually a Hilbert space of the solutions of the equation $A u=0$ in a domain $U \subset X$.

Let the operator $A$ be included into an elliptic compatibility complex of differential operators $A^{i} \in \operatorname{Diff}^{m_{i}}\left(X ; E^{i}, E^{i+1}\right), i=0,1, \ldots, N$, over $X$, with $A^{0}=A$.

Suppose that $V^{i} \hookrightarrow \mathscr{D}^{\prime}\left(\stackrel{\circ}{X}, E^{i}\right), i=0,1, \ldots, N$, are Hilbert spaces of the sections of $E^{i}$ over $X$, such that

(1) $V^{i} \cap C^{\infty}\left(X, E^{i}\right)$ is dense in $V^{i}$ for all $i=0,1, \ldots, N$;

(2) $A^{i}$ maps $V^{i} \cap C^{\infty}\left(X, E^{i}\right)$ to $V^{i+1} \cap C^{\infty}\left(X, E^{i+1}\right)$.

Let $\mathscr{D}_{T}^{i}$ be the set of all sections $u \in V^{i}$ for which there is a sequence $\left\{u_{v}\right\}$ with the following properties:

(1) $u_{v} \in V^{i} \cap C^{\infty}\left(X, E^{i}\right)$;

(2) $\left\{u_{v}\right\}$ converges to $u$ in $V^{i}$;

(3) $\left\{A u_{v}\right\}$ is a Cauchy sequence in $V^{i+1}$.

The mapping $T: \mathscr{D}_{T}^{i} \rightarrow V^{i+1}$ defined by $T u=\lim A u_{v}$, where $\left\{u_{v}\right\}$ is a sequence with properties (1), (2), and, (3), is called the maximal operator generated by $A$.

Note that $T$ is well defined. Indeed, if $\left\{u_{v}^{\prime}\right\}$ is another sequence satisfying (1), (2), and (3), and $f=\lim A u_{v}^{\prime}$, then, for all $g \in C^{\infty}\left(X, E^{i+1 *}\right)$ with a compact support in the interior of $X$, we get

$$
\langle T u-f, g\rangle=\lim \left\langle A u_{v}-A u_{v}^{\prime}, g\right\rangle=\lim \left\langle u_{v}-u_{v}^{\prime}, A^{\prime} g\right\rangle=0,
$$

whence $T u=f$.

We think of $T$ as an unbounded operator from $V^{i}$ to $V^{i+1}$ whose domain is $\mathscr{D}_{T}^{i}$. Since $\mathscr{D}_{T}^{i}$ contains $V^{i} \cap C^{\infty}\left(X, E^{i}\right)$, the operator $T$ is densely defined and closed.

From the lemma of Du Bois-Reymond and the uniqueness of a weak limit, it follows that if $u \in \mathscr{D}_{T}^{i}$ then $T u=A u$ in the sense of distributions in the interior of $X$.

LEMMA 2.8. As defined above, $T$ satisfies $T \mathscr{D}_{T}^{i} \subset \mathscr{D}_{T}^{i+1}$ and $T^{2}=0$.

Proof. Let $u \in \mathscr{D}_{T}^{i}$ and $\left\{u_{v}\right\}$ be a sequence with properties (1), (2), and (3). We set $f_{v}=A u_{v}$. Then, $T u=\lim f_{v}$, and, since $A f_{v}=0$, we obtain that $T u \in \mathscr{D}_{T}^{i+1}$ and $T(T u)=0$.

Thus, we have the following complex of Hilbert spaces and their closed linear mappings:

$$
V^{\cdot}: 0 \longrightarrow V^{0} \stackrel{T}{\longrightarrow} V^{1} \stackrel{T}{\longrightarrow} \cdots \stackrel{T}{\longrightarrow} V^{N} \longrightarrow 0 .
$$

The cohomology of the complex $\left\{E^{i}, A^{i}\right\}$ evaluated by the spaces $\left\{V^{i}\right\}$ is just the cohomology of complex (2.30), that is,

$$
H^{i}\left(V^{\cdot}\right)=\frac{\operatorname{ker}\left\{T: \mathscr{D}_{T}^{i} \longrightarrow V^{i+1}\right\}}{T \mathscr{D}_{T}^{i-1}} .
$$


We now define $T^{*}$, the adjoint of $T$, as usual for unbounded operators. Namely, let $\mathscr{D}_{T^{*}}^{i}$ be the set of all $g \in V^{i}$ with the property that there is $v \in V^{i-1}$ satisfying $(T u, g)_{V^{i}}=(u, v)_{V^{i-1}}$ for all $u \in \mathscr{D}_{T}^{i-1}$. We define $T^{*}: \mathscr{D}_{T^{*}}^{i} \rightarrow V^{i-1}$ by $T^{*} g=v$.

The operator $T^{*}$ is well defined because the domain $\mathscr{D}_{T}^{i-1}$ is dense in $V^{i-1}$. It is clear that $T^{*} g$ is in general different from $A^{*} g$ in the sense of distributions in the interior of $X$, for $A^{*}$ is formally adjoint for $A$ in the sense of $L^{2}$-spaces on $X$.

LEMMA 2.9. The operator $T^{*}$ satisfies $T^{*} \mathscr{D}_{T^{*}}^{i} \subset \mathscr{D}_{T^{*}}^{i-1}$ and $T^{* 2}=0$.

Proof. Indeed, if $g \in \mathscr{D}_{T^{*}}^{i}$ and $u \in \mathscr{D}_{T}^{i-2}$, then by definition and Lemma 2.8 we get

$$
\left(T u, T^{*} g\right)_{V^{i-1}}=(T(T u), g)_{V^{i}}=0 .
$$

Therefore, $T^{*} g \in \mathscr{D}_{T^{*}}^{i-1}$ and $T^{*}\left(T^{*} g\right)=0$, which completes the proof.

Thus, we obtain the following (chain) complex of Hilbert spaces and their closed linear mappings:

$$
V^{*}: 0 \longleftarrow V^{0} \stackrel{T^{*}}{\longleftarrow} V^{1} \stackrel{T^{*}}{\longleftarrow} \cdots \stackrel{T^{*}}{\longleftarrow} V^{N} \leftarrow-0 .
$$

The complex (2.33) is called the adjoint complex for (2.30), and its homology is denoted by

$$
H_{i}\left(V^{*}\right)=\frac{\operatorname{ker}\left\{T^{*}: \mathscr{D}_{T^{*}}^{i} \longrightarrow V^{i-1}\right\}}{T^{*} \mathscr{D}_{T^{*}}^{i+1}} .
$$

Introduce an operator $L$ on $V^{i}$ with a domain $\mathscr{D}_{L}^{i}$, which better suits the Hilbert structure of $V^{i}$ than the formal Laplacian $\Delta=A^{*} A+A A^{*}$ of the complex $\left\{E^{i}, A^{i}\right\}$. Namely, write $\mathscr{D}_{L}^{i}$ for the set of all $u \in \mathscr{D}_{T}^{i} \cap \mathscr{D}_{T^{*}}^{i}$ with the property that $T u \in \mathscr{D}_{T^{*}}^{i+1}$ and $T^{*} u \in \mathscr{D}_{T}^{i-1}$. Then, the operator $L: \mathscr{D}_{L}^{i} \rightarrow V^{i}$ is defined by

$$
L u=T^{*} T u+T T^{*} u
$$

(cf. [22, Section 4.2]).

The Neumann problem for the complex $\left\{E^{i}, A^{i}\right\}$ in the spaces $V^{i}$ consists in the following:

(NP) given a section $f \in V^{i}$, when is there $u \in \mathscr{D}_{L}^{i}$ such that $L u=f$ ? And how does $u$ depend on $f$ ?

The weak orthogonal decomposition is actually the first step in solving the Neumann problem. Set

$$
\mathscr{H}^{i}=\left\{u \in \mathscr{D}_{T}^{i} \cap \mathscr{D}_{T^{*}}^{i}: T u=T^{*} u=0\right\}
$$


for $i=0,1, \ldots$. Since the operators $T$ and $T^{*}$ are closed, $\mathscr{H}^{i}$ is a closed subspace of $V^{i}$. Denote by $H: V^{i} \rightarrow \mathscr{H}^{i}$ the orthogonal projection of $V^{i}$ onto $\mathscr{H}^{i}$.

LEMMA 2.10. Let $u \in V^{i}$. Then $u \in \mathscr{H}^{i}$ if and only if $u \in \mathscr{D}_{L}^{i}$ and $L u=0$.

Proof. If $u \in \mathscr{H}^{i}$, then obviously $u \in \mathscr{D}_{L}^{i}$ and $L u=0$. If $L u=0$, then $(L u, u)_{V^{i}}=0$, and since

$$
(L u, u)_{V^{i}}=\|T u\|_{V^{i+1}}^{2}+\left\|T^{*} u\right\|_{V^{i-1}}^{2},
$$

we have $u \in \mathscr{H}^{i}$.

LEMMA 2.11. The operator $L$ is selfadjoint, and $(L+1)^{-1}$ exists, is bounded, and is defined in $V^{i}$ everywhere.

Proof. Since $T$ is a closed operator and the domain of $T$ is dense, the same is also true for $T^{*}$, and $\left(T^{*}\right)^{*}=T$.

It follows that the operators $\left(T T^{*}+1\right)^{-1}$ and $\left(T^{*} T+1\right)^{-1}$ exist, are bounded, selfadjoint, and defined everywhere in $V^{i}$ (cf. [24, page 200]).

We now easily verify that $(L+1)^{-1}$ exists, is bounded, is defined everywhere, and is given by the formula

$$
(L+1)^{-1}=\left(T T^{*}+1\right)^{-1}+\left(T^{*} T+1\right)^{-1}-1
$$

(cf. [22, Section 4.2.4] and elsewhere).

COROLLARY 2.12 (weak orthogonal decomposition). The range of $L$ is orthogonal to $\mathcal{H}^{i}$, and

$$
V^{i}=\mathscr{H}^{i} \oplus \overline{L_{\mathscr{D}}^{i}}
$$

where $\overline{L_{L}^{i}}$ denotes the closure of $L \mathscr{D}_{L}^{i}$ in $V^{i}$.

Proof. This follows immediately from the selfadjointness of $L$ and Lemma 2.10 .

In particular, if $L \mathscr{D}_{L}^{i}$ is closed, then we get the strong orthogonal decomposition

$$
V^{i}=\mathscr{H}^{i} \oplus T^{*} T \mathscr{D}_{L}^{i} \oplus T T^{*} \mathscr{D}_{L}^{i}
$$

DEFINITION 2.13. Let $L \mathscr{D}_{L}^{i}$ be closed and $f \in V^{i}$, then $f=H f+L u$ where $u \in \mathscr{D}_{L}^{i}$. The Neumann operator $N: V^{i} \rightarrow \mathscr{D}_{L}^{i}$ is defined by $N f=u-H u$.

Note that $N$ is well defined. Indeed, if also $f=H f+L u^{\prime}$ where $u^{\prime} \in \mathscr{D}_{L}^{i}$, then $L\left(u-u^{\prime}\right)=0$, whence

$$
(u-H u)-\left(u^{\prime}-H u^{\prime}\right)=\left(u-u^{\prime}\right)-H\left(u-u^{\prime}\right)=0 .
$$


We summarize the properties of the Neumann operator. They generalise those of the Green operator from Hodge theory, for the Neumann problem itself stems from the desire to extend the Hodge theory to the case of manifolds with boundary.

LEMmA 2.14. Suppose that $L \mathscr{D}_{L}^{i}$ is closed. Then, the Neumann operator $N$ has the following properties:

(1) $N$ is bounded, selfadjoint, $H N=N H=0$, and we have the orthogonal decomposition

$$
f=H f+T^{*} T N f+T T^{*} N f
$$

for all $f \in V^{i}$;

(2) if $f \in \mathscr{D}_{T}^{i}$ and $T f=0$, then $T N f=0$. If, moreover, $L \mathscr{D}_{L}^{i+1}$ is closed, then $T N f=N T f$

(3) if $f \in \mathscr{D}_{T^{*}}^{i}$ and $T^{*} f=0$, then $T^{*} N f=0$. If, moreover, $L \mathscr{D}_{L}^{i-1}$ is closed, then $T^{*} N f=N T^{*} f$.

Proof. (1) The equalities $H N=N H=0$ and formula (2.42) follow immediately from the definition of $N$.

Further, by the closed graph theorem, there exists a constant $c>0$ such that if $u \in \mathscr{D}_{L}^{i}$ is orthogonal to $\mathscr{H}^{i}$ then we have $\|L u\| \geq c\|u\|$. Applying this to $N f$, we obtain

$$
\|N f\| \leq \frac{1}{c}\|L N f\|=\frac{1}{c}\|f-H f\| \leq \frac{1}{c}\|f\| .
$$

Hence $N$ is bounded.

Finally, the selfadjointness of $N$ follows immediately from Lemma 2.11 because

$$
\begin{aligned}
(N f, g)_{V^{i}} & =(N f, H g+L N g)_{V^{i}}=(N f, L N g)_{V^{i}} \\
& =(L N f, N g)_{V^{i}}=(f, N g)_{V^{i}} .
\end{aligned}
$$

(2) Let $f \in \mathscr{D}_{L}^{i}$. Then from (2.42) and Lemma 2.8, we get $T^{*} T N f \in \mathscr{D}_{T}^{i}$ and $T f=0$ implies $T T^{*} T N f=0$. Hence, it easily follows that $T N f=0$.

If also $L \mathscr{D}_{L}^{i+1}$ is closed, then for any $f \in \mathscr{D}_{T}^{i}$ we have $T f=T T^{*} T N f$ on the one hand, and $T f=T T^{*} N T f$ on the other hand. Hence, it follows that $L(T N f-N T f)=0$, and, since $T N f-N T f$ is orthogonal to $\mathscr{H}^{i+1}$, we deduce that $T N f-N T f=0$, as required.

(3) The proof is analogous to that of part (2).

If $L^{i}$ is a hypoelliptic pseudodifferential operator in the interior of $X$, then the harmonic space $\mathscr{H}^{i}$ consists of $C^{\infty}$ sections in the interior of $X$ and the Neumann operator $N$ preserves the interior regularity. Such is the case, in particular, if $V^{i}$ are Sobolev spaces. 
Beginning with its classical forms, the Dirichlet norm has been an important technical tool in studying the Neumann problem. by

Given any $u, v \in \mathscr{D}_{T}^{i} \cap \mathscr{D}_{T^{*}}^{i}$, the Dirichlet inner product of $u$ and $v$ is defined

$$
D(u, v)=(T u, T v)_{V^{i+1}}+\left(T^{*} u, T^{*} v\right)_{V^{i-1}}+(u, v)_{V^{i}}
$$

and the Dirichlet norm is $D(u)=\sqrt{D(u, u)}$.

The space $\mathscr{D}_{T}^{i} \cap \mathscr{D}_{T^{*}}^{i}$ with the Dirichlet norm is a complete (Hilbert) space. It is denoted by $\mathscr{D}^{i}$.

Since $D(u) \geq\|u\|_{V^{i}}$ for all $u \in \mathscr{D}^{i}$, there exists only one selfadjoint operator $S$ with a domain $\mathscr{D}_{S}^{i} \subset \mathscr{D}^{i}$, such that if $u \in \mathscr{D}_{S}^{i}$ and $v \in \mathscr{D}^{i}$ then

$$
D(u, v)=(S u, v)_{V^{i}}
$$

The following lemma gives a useful description of the operator $L$ because our estimates will be in the norm $D(u)$.

LEMMA 2.15. The equalities hold $\mathscr{D}_{L}^{i}=\mathscr{D}_{S}^{i}$ and $L=S-1$, where the operator $S$ is defined by (2.46).

Proof. If $u \in \mathscr{D}_{L}^{i}$ and $v \in \mathscr{D}^{i}$, then $D(u, v)=((L+1) u, v)_{V^{i}}$ is fulfilled. Hence by the uniqueness of $S$, we have $S=L+1$.

Let $\|\cdot\|_{1}$ and $\|\cdot\|_{2}$ be two norms on a vector space $V$. We say that the norm $\|\cdot\|_{1}$ is completely continuous with respect to the norm $\|\cdot\|_{2}$ if every sequence which is bounded in the norm $\|\cdot\|_{1}$ has a convergent subsequence in the norm $\|\cdot\|_{2}$.

LEMMA 2.16. If the norm $D$ on $\mathscr{D}^{i}$ is completely continuous with respect to $\|\cdot\|_{V^{i}}$, then $\dot{H}^{i}$ is finite dimensional.

Proof. Observe that if $u, v \in \mathscr{H}^{i}$ then $D(u, v)=(u, v)_{V^{i}}$. Suppose that the dimension of $\mathscr{H}^{i}$ is infinite. Then there exists an infinite sequence $\left\{u_{v}\right\}$ of orthonormal elements in $\mathscr{H}^{i}$. Since $D\left(u_{v}\right)=\left\|u_{v}\right\|_{V^{i}}=1$, the sequence $\left\{u_{v}\right\}$ contains a convergent subsequence. But this is at variance with the fact that if $v \neq \mu$ then $\left\|u_{v}-u_{\mu}\right\|_{V^{i}}=\sqrt{2}$.

LEMMA 2.17. If the norm $D$ on $\mathscr{D}^{i}$ is completely continuous with respect to $\|\cdot\|_{V^{i}}$, then there exists a constant $c>0$ such that, for all $u \in D^{i}$ orthogonal to $\mathscr{H}^{i}$,

$$
\|T u\|_{V^{i+1}}^{2}+\left\|T^{*} u\right\|_{V^{i-1}}^{2} \geq c\|u\|_{V^{i}}^{2}
$$

Proof. Consider the Hilbert space $V^{i+1} \times V^{i-1}$ which is equipped with the norm

$$
\|\{f, v\}\|=\left(\|f\|_{V^{i+1}}^{2}+\|v\|_{V^{i-1}}^{2}\right)^{1 / 2}
$$


Let $2 \mathfrak{V}: \mathscr{D}^{i} \rightarrow V^{i+1} \times V^{i-1}$ be the mapping defined by $2 \mathfrak{W} u=\left\{T u, T^{*} u\right\}$. We note that $2 \mathfrak{W}$ is a closed operator.

We prove that the range of $2 \mathfrak{b}$ is closed. Suppose that $2 \mathfrak{b} \mathscr{D}^{i}$ is not closed. Then there exists a sequence $\left\{u_{v}\right\}$ in $\mathscr{D}^{i}$ such that $\lim 2 \mathfrak{D} u_{v}=\{f, v\}$ and $\{f, v\} \notin$ 2nDi ${ }^{i}$.

Set $u_{v}^{\prime}=u_{v}-H u_{v}$, then $u_{v}^{\prime}$ are orthogonal to $\mathscr{H}^{i}$ and $\lim 2 \mathfrak{D} u_{v}^{\prime}=\{f, v\}$. If $\left\|u_{v}^{\prime}\right\|_{V^{i}}$ are bounded, then $D\left(u_{v}^{\prime}\right)=\left(\left\|2 b u_{v}^{\prime}\right\|^{2}+\left\|u_{v}^{\prime}\right\|_{V^{i}}^{2}\right)^{1 / 2}$ are bounded, too. Then, by hypothesis, $\left\{u_{v}^{\prime}\right\}$ has a convergent subsequence with a limit $u$, and since $2 \mathfrak{W}$ is closed then $2 \mathfrak{b u}=\{f, v\}$, which contradicts the assumption that $\{f, v\} \notin 2 \mathfrak{D} \mathscr{D}^{i}$. Thus by choosing a subsequence, if necessary, we may assume that $\lim \left\|u_{v}^{\prime}\right\|_{V^{i}}=\infty$.

Now set $U_{v}=u_{v}^{\prime} /\left\|u_{v}^{\prime}\right\|_{V^{i}}$. Then $\lim \left\|20 U_{v}\right\|=0$ and $D\left(U_{v}\right)$ are bounded. Therefore, $\left\{U_{v}\right\}$ has a convergent subsequence $\left\{U_{v_{k}}\right\}$ such that

$$
\lim U_{v_{k}}=U, \quad \lim 2 \mathfrak{n} U_{v_{k}}=\{0,0\} .
$$

Hence $2 \mathfrak{b} U=0$ so that $U \in \mathscr{H}^{i}$. Since $U_{v}$ is orthogonal to $\mathscr{H}^{i}$, we have $U=0$, but $\left\|U_{V}\right\|_{V^{i}}=1$. This contradiction proves that the range $20 \mathscr{D}^{i}$ is closed in $V^{i+1} \times V^{i-1}$.

Let $R$ be the restriction of $2 \mathfrak{W}$ to the orthogonal complement of $\mathscr{H}^{i}$ in $\mathscr{D}^{i}$. Then $R$ is one-to-one and has a closed range. By the closed graph theorem, the inverse $R^{-1}$ is bounded. Hence there is $c>0$ such that $\|R u\|^{2} \geq c\|u\|_{V^{i}}^{2}$. This proves the lemma.

THEOREM 2.18. If the norm $D$ on $\mathscr{D}^{i}$ is completely continuous with respect to the norm $\|\cdot\|_{V^{i}}$, then $L_{D^{i}}$ is closed.

Proof. By Lemma 2.17, there exists $c>0$ with the property that, for all $u \in \mathscr{D}_{L}^{i}$ which are orthogonal to $\mathscr{H}^{i}$, we have

$$
(L u, u)_{V^{i}} \geq c\|u\|_{V^{i}}^{2}
$$

so that $\|L u\|_{V^{i}} \geq c\|u\|_{V^{i}}$.

Set $f=\lim L u_{v}$. We may assume that $u_{v}$ are orthogonal to $\mathscr{H}^{i}$, and then $\left\|u_{v}\right\|_{V^{i}}$ are uniformly bounded. Therefore, $\left\{u_{v}\right\}$ has a subsequence whose arithmetic means converge. (This actually puts some restrictions on the spaces $V^{i}$ under study.) Denoting this limit by $u$, we get $f=L u$, which completes the proof.

The question of when the norm $D$ on $\mathscr{D}^{i}$ is completely continuous with respect to the norm $\|\cdot\|_{V^{i}}$ is very difficult in the general case, and it requires special consideration. We present some consequences here.

COROLLARY 2.19. Suppose that the norm $D$ on $\mathscr{D}^{i}$ is completely continuous with respect to the norm $\|\cdot\|_{V^{i}}$. Then, for the complex $\left\{E^{i}, A^{i}\right\}$, the Neumann problem is solvable at step $i$ in the sense that there exist operators $H$ and $N$ in $V^{i}$ with properties (1), (2), and (3) of Lemma 2.14. 
Proof. This follows immediately from Lemma 2.14 and Theorem 2.18.

For compact manifolds with boundary $X$, the subspace $\mathscr{H}^{0}$ is usually infinite dimensional so, by Lemma 2.16, the Dirichlet norm $D$ may not be completely continuous with respect to the norm $\|\cdot\|_{V^{0}}$ on $\mathscr{D}^{0}$. But the following result holds.

THEOREM 2.20. If the norm $D$ on $\mathscr{D}^{1}$ is completely continuous with respect to the norm $\|\cdot\|_{V^{1}}$, then $L \mathscr{D}_{L}^{0}$ is closed.

Proof. It suffices to prove that there exists a constant $c>0$ such that $\|L f\|_{V^{0}} \geq c\|f\|_{V^{0}}$ for all $f \in \mathscr{D}_{L}^{0}$ which are orthogonal to $\mathscr{H}^{0}$.

First, if $u \in \mathscr{D}_{L}^{0}$, then $T u \in \mathscr{D}^{1}$ and $T u \perp \mathscr{H}^{1}$. Thus by Lemma 2.17, we obtain

$$
\left\|T^{*} T u\right\|_{V^{0}}^{2}=\|L u\|_{V^{0}}^{2} \geq c\|T u\|_{V^{1}}^{2}
$$

Further, since $f \perp \mathscr{H}^{0}$, then, by the weak orthogonal decomposition (2.39), $f \in \overline{L \mathscr{D}_{L}^{0}}$. Hence, for each $\varepsilon>0$, there exists $u \in \mathscr{D}_{L}^{0}$ such that $\|f-L u\|_{V^{0}}<\varepsilon$. Thus,

$$
\begin{aligned}
\|f\|_{V^{0}}^{2} & \leq(L u, f)_{V^{0}}+\varepsilon\|f\|_{V^{0}} \\
& \leq\|T u\|_{V^{1}}\|T f\|_{V^{1}}+\varepsilon\|f\|_{V^{0}} \\
& \leq \frac{1}{c}\|L u\|_{V^{0}}\|L f\|_{V^{0}}+\varepsilon\|f\|_{V^{0}} \\
& \leq \frac{1}{c}\|f\|_{V^{0}}\|L f\|_{V^{0}}+\varepsilon\left(\frac{1}{c}\|L f\|_{V^{0}}+\|f\|_{V^{0}}\right) .
\end{aligned}
$$

Since $\varepsilon$ can be made arbitrarily small by choosing $L u$ close enough to $f$, we obtain $\|L f\|_{V^{0}} \geq c\|f\|_{V^{0}}$, which concludes the proof.

The next result follows from Lemma 2.14 and Theorem 2.18. Recall that $\mathscr{H}^{0}=\operatorname{ker} T^{0}$.

COROLLARY 2.21. Suppose that the norm $D$ on $\mathscr{D}^{1}$ is completely continuous with respect to the norm $\|\cdot\|_{V^{1}}$. Then, $f=H f+T^{*} N T f$ for any section $f \in \mathscr{D}_{T}^{0}$, where $H: V^{0} \rightarrow \mathscr{H}^{0}$ is the orthogonal projection.

By assumption, the differential operator $A^{0}=A$ has injective symbol. It follows that $A^{0}$ is hypoelliptic in the interior of $X$ whence

$$
\mathscr{H}^{0}=\left\{u \in V^{0} \cap C_{\mathrm{loc}}^{\infty}\left(\stackrel{\circ}{X}, E^{0}\right): A u=0\right\},
$$

that is, the operator $H^{0}$ is a generalisation of the Bergman projector from complex analysis. Corollary 2.21 gives

$$
\pi:=H^{0}=I-T^{*} N T .
$$


As mentioned, a priori estimates for solutions of elliptic equations imply that, for each interior point $x$ of $X$, the evaluation functional $\delta_{x}(u)=u(x)$ is bounded on $\mathscr{H}^{0}$. Therefore, $\mathscr{H}^{0}$ is a Hilbert space with reproducing kernel (cf. [1]).

Let $\left\{e_{v}\right\}_{v=1,2, \ldots}$ be some complete orthonormal system in $\mathscr{H}^{0}$. If $u \in \mathscr{H}^{0}$, then this section decomposes into the Fourier series $u=\sum c_{v} e_{v}$ which converges in the norm of the space $V^{0}$ and hence uniformly along with all derivatives on compact subsets of the interior of $X$. In the interior of $X \times X$, we consider the series

$$
K(x, y)=K_{H}(x, y)=\sum_{v=0}^{\infty} *_{E} e_{v}(x) \otimes e_{v}(y) .
$$

THEOREM 2.22. Series (2.55) converges uniformly along with all derivatives on compact subsets of the interior of $X \times X$, so that

$$
K_{H} \in C_{\mathrm{loc}}^{\infty}\left(\stackrel{\circ}{X} \times \stackrel{\circ}{X}, E^{*} \otimes E\right) .
$$

If $x \in \stackrel{\circ}{X}$ is fixed, then this series actually converges in the norm of the space $E_{x}^{*} \otimes V^{0}$.

Proof. To shorten notation, we will restrict the discussion to the case where $X$ is a closed domain in $\mathbb{R}^{n}$.

Let

$$
e_{\nu}(x)=\left(\begin{array}{c}
e_{\nu, 1}(x) \\
\vdots \\
e_{\nu, k}(x)
\end{array}\right)
$$

be representations of the sections $e_{v}$, where $k$ is the rank of $E$. Pick compact sets $K_{1}$ and $K_{2}$ in the interior of $X$. If $x \in K_{1}$ is a fixed point, then, in view of the orthonormality of the system $\left\{e_{v}\right\}$, we obtain for $j=1, \ldots, k$

$$
\begin{aligned}
\left(\sum_{v=0}^{N}\left|e_{v, j}(x)\right|^{2}\right)^{2} & \leq\left|\sum_{v=0}^{N} \overline{e_{v, j}(x)} e_{v}(x)\right|^{2} \\
& \leq\left. c_{1}|| \sum_{v=0}^{N} \overline{e_{v, j}(x)} e_{v}(y)\right|_{V^{0}} ^{2} \\
& =c_{1} \sum_{v=0}^{N}\left|e_{v, j}(x)\right|^{2},
\end{aligned}
$$


the constant $c_{1}>0$ depending on $A$ and $K$ only. Hence

$$
\sum_{v=0}^{N}\left|e_{v, j}(x)\right|^{2} \leq c_{1}
$$

for all $x \in K_{1}$.

Therefore, denoting by $c_{2}$ the constant obtained by analogy for the set $K_{2}$, we get for $(x, y) \in K_{1} \times K_{2}$

$$
\sum_{\nu=0}^{N}\left|*_{E} e_{\nu}(x) \otimes e_{\nu}(y)\right|=\sum_{\nu=0}^{N}\left|e_{\nu}(x)\right|\left|e_{\nu}(y)\right| \leq k \sqrt{c_{1} c_{2}} .
$$

This proves the absolute and uniform convergence of series (2.55) on compact subsets of the interior of $X \times X$.

Finally, (2.59) implies that, for fixed $x \in \stackrel{\circ}{X}$, equality (2.55) gives the expansion of $K(x, y)$ in the complete orthonormal system $\left\{e_{v}\right\}$. To finish the proof, it is sufficient to observe that $x$ and $y$ enter into $K(x, y)$ in a symmetric way.

THeOrem 2.23 (Bergman formula). If $u \in \mathscr{H}^{0}$, then

$$
u(x)=(u, K(x, \cdot))_{V^{0}}
$$

for all $x \in \stackrel{\circ}{X}$.

Proof. Let $u=\sum c_{\mu} e_{\mu}$. Then, by the previous theorem, we get for fixed $x$ in the interior of $X$

$$
(u, K(x, \cdot))_{V^{0}}=\sum_{\mu, v} c_{\mu}\left(e_{\mu}, e_{v}\right)_{V^{0}} e_{v}(x)=\sum_{v} c_{v}\left(e_{v}, e_{v}\right)_{V^{0}} e_{v}(x)=u(x),
$$

and the proof is complete.

Thus, in order to discover the properties of $\pi=H$, we might study the Neumann operator $N^{1}$. However, "good" properties of $N^{1}$ is not what we can generally expect. It is rather an instrument to produce examples for the general scheme.

2.4. Hodge theory on manifolds with boundary. Given a vector space $V$ with norm $\|\cdot\|$, we write $C(V,\|\cdot\|)$ for the completion of $V$ under the norm $\|\cdot\|$.

In this section, we describe the Hodge theory of the Dirichlet problem for the Laplacian $\Delta=A^{*} A$ in the class of generalised sections of $E$ on $X$. In order to define what is meant by the solution of the boundary value problem, we employ negative norms. This idea is certainly not new and goes back at least as far as $[13,14]$. 
Pick a Dirichlet system $B_{j}, j=0,1, \ldots, m-1$, of order $m-1$ on the boundary of $X$. More precisely, $B_{j}$ is a differential operator of type $E \rightarrow F_{j}$ and order $m_{j} \leq m-1$ in a neighbourhood $U$ of $\partial X$. Moreover, the orders $m_{j}$ are pairwise different and the symbols $\sigma\left(B_{j}\right)$, if restricted to the conormal bundle of $\partial X$, have ranks equal to the dimensions of $F_{j}$.

We actually assume that the dimensions of $F_{j}$ are the same and equal to that of $E$.

Let $C_{j}, j=0,1, \ldots, m-1$, be the adjoint system for $\left\{B_{j}\right\}$ with respect to Green's formula (cf. [21]). Thus, $C_{j}$ is a differential operator of type $F^{*} \rightarrow F_{j}^{*}$ and order $m-m_{j}-1$ in a smaller neighbourhood $U$ of $\partial X$. We now set

$$
t(u)=\bigoplus_{j=0}^{m-1} B_{j} u, \quad n(f)=\bigoplus_{j=0}^{m-1} *_{F_{j}}^{-1} C_{j} *_{F}
$$

for $u \in C_{\mathrm{loc}}^{m-1}(U, E)$ and $f \in C_{\mathrm{loc}}^{m-1}(U, F)$.

LEMMA 2.24 (Green's formula). For each $u, v \in H^{2 m}(X, E)$, the following formula holds:

$$
\int_{\partial X}\left((t(u), n(A v))_{x}-(n(A u), t(v))_{x}\right) d s=\int_{X}\left((\Delta u, v)_{x}-(u, \Delta v)_{x}\right) d x
$$

Proof. See [21, Corollary 9.2.12].

Given $F$, we consider the boundary value problem

$$
\Delta u=F \quad \text { in } X, \quad t(u)=0 \quad \text { on } \partial X,
$$

which is an obvious generalisation of the classical Dirichlet problem (cf. [21, Section 9.2.4]).

Suppose that $s>0$. For sections $u \in C^{\infty}(X, E)$ we define two types of negative norms

$$
\|u\|_{-s}=\sup _{v \in C^{\infty}(X, E)} \frac{|(u, v)|}{\|v\|_{s}}, \quad|u|_{-s}=\sup _{\substack{v \in C^{\infty}(X, E) \\ t(v)=0}} \frac{|(u, v)|}{\|v\|_{s}},
$$

where $(\cdot, \cdot)$ is the scalar product in $L^{2}(X, E)$. We denote the completions of $C^{\infty}(X, E)$ with respect to these norms by $H^{-s}(X, E)$ and $C\left(C^{\infty}(X, E),|\cdot|{ }_{-s}\right)$, respectively. They are obviously Banach spaces and satisfy

$$
H^{-s}(X, E) \longleftrightarrow C\left(C^{\infty}(X, E),|\cdot|_{-s}\right),
$$

for $\|u\|_{-s} \geq|u|_{-s}$. 
We can define $(u, v)$ for $u \in H^{-s}(X, E)$ and $v \in C^{\infty}(X, E)$ as follows. By definition, there is a sequence $\left\{u_{v}\right\}$ in $C^{\infty}(X, E)$ such that $\left\|u_{v}-u\right\|_{-s} \rightarrow 0$ as $v \rightarrow \infty$. Then

$$
\left|\left(u_{v}-u_{\mu}, v\right)\right| \leq\left\|u_{v}-u_{\mu}\right\|_{-s}\|v\|_{s} \rightarrow 0
$$

as $\mu, v \rightarrow \infty$. Set $(u, v)=\lim \left(u_{v}, v\right)$. Clearly, this limit does not depend on the particular sequence $\left\{u_{v}\right\}$, for if $\left\|u_{v}\right\|_{-s} \rightarrow 0$, then $\left|\left(u_{v}, v\right)\right| \leq\left\|u_{v}\right\|_{-s}\|v\|_{s}$ tends to zero, too. From the definition, it follows that for all $u \in H^{-s}(X, E)$ and $v \in C^{\infty}(X, E)$, we get

$$
|(u, v)| \leq\|u\|_{-s}\|v\|_{s}
$$

In a similar way, we can define the pairing $(u, v)$ for $u \in C\left(C^{\infty}(X, E),|\cdot|_{-s}\right)$ and $v \in C^{\infty}(X, E)$ with $t(v)=0$. Corresponding to (2.69), we obviously have $|(u, v)| \leq|u|_{-s}\|v\|_{s}$.

Let $F$ be in $C\left(C^{\infty}(X, E),|\cdot|_{-s-2 m}\right)$, where $s \geq 0$. We say that $u \in H^{-s}(X, E)$ is a strong solution of (2.65) if there is a sequence of sections $u_{v} \in C^{\infty}(X, E)$ with $t\left(u_{v}\right)=0$, such that

$$
\left\|u_{v}-u\right\|_{-s} \longrightarrow 0, \quad\left|\Delta u_{v}-F\right|_{-s-2 m} \longrightarrow 0
$$

as $v \rightarrow \infty$.

Denote by $\mathscr{H}(X)$ the set of all $u \in C^{\infty}(X, E)$ that satisfy $\Delta u=0$ in the interior of $X$ and $t(u)=0$ on $\partial X$. Since (2.65) is an elliptic boundary value problem, $\mathscr{H}(X)$ is finite dimensional. Moreover, for any $u \in \mathscr{H}(X)$, we actually obtain

$$
0=(\Delta u, u)=(A u, A u)
$$

whence $A u=0$ in $X$. Therefore, the space $\mathscr{H}(X)$ consists of all $u \in \mathscr{Y}_{A}(\stackrel{\circ}{X})$ which are $C^{\infty}$ up to the boundary of $X$ and which vanish up to the infinite order on $\partial X$.

LEMMA 2.25. Let $s \geq 0$. If $F \in C\left(C^{\infty}(X, E),|\cdot|_{-s-2 m}\right)$ and $F \perp \mathscr{H}(X)$, then there is a strong solution $u \in H^{-s}(X, E)$ of (2.65) satisfying $u \perp \mathscr{H}(X)$ and

$$
\|u\|_{-s} \leq c|F|_{-s-2 m},
$$

where the constant $c$ does not depend on $F$ and $u$.

Proof. See [14].

Definition (2.70) of a strong solution of (2.65) obviously corresponds to an appropriate closure $L: \mathscr{D}_{L} \rightarrow C\left(C^{\infty}(X, E),|\cdot|_{-s-2 m}\right)$ of the Laplacian $\Delta=A^{*} A$ (cf. [5, Chapter 2]). Namely, we denote by $\mathscr{D}_{L}$ the set of all sections $u \in H^{-s}(X, E)$, for which there is a sequence $\left\{u_{v}\right\}$ with the following properties:

(1) $u_{v} \in C^{\infty}(X, E)$ satisfies $t\left(u_{v}\right)=0$; 
(2) $\left\{u_{v}\right\}$ converges to $u$ in $H^{-s}(X, E)$;

(3) $\left\{\Delta u_{v}\right\}$ is a Cauchy sequence in $C\left(C^{\infty}(X, E),|\cdot|_{-s-2 m}\right)$.

The closed densely defined operator $L: \mathscr{D}_{L} \rightarrow C\left(C^{\infty}(X, E),|\cdot|_{-s-2 m}\right)$ given by $L u=\lim \Delta u_{v}$, where $\left\{u_{v}\right\}$ is any sequence with properties (1), (2), and (3), is called the strong extension of $\Delta$ under the boundary conditions $t(u)=0$. It is clear that $u \in H^{-s}(X, E)$ is a strong solution to problem (2.65) if and only if $L u=F$.

It is worth pointing out that the case $\partial X=\varnothing$ is formally permitted in the following theorem.

THEOREM 2.26. Suppose that $s \geq 0$. There are bounded linear operators

$$
H: C\left(C^{\infty}(X, E),|\cdot|_{-s-2 m}\right) \longrightarrow \mathscr{H}(X), \quad G: C\left(C^{\infty}(X, E),|\cdot|_{-s-2 m}\right) \longrightarrow \mathscr{D}_{L}
$$

such that

(1) $H$ has the kernel $K_{H}(x, y)=\sum_{v} h_{v}(x) \otimes *_{E} h_{v}(y)$, where $\left\{h_{v}\right\}$ is an orthonormal basis of $\mathscr{H}(X)$;

(2) $A H=0$ and $G H=H G=0$;

(3)

$$
\begin{array}{ll}
G L u=u-H u & \text { for all } u \in \mathscr{D}_{L}, \\
L G F=F-H F & \text { for all } F \in C\left(C^{\infty}(X, E),|\cdot|_{-s-2 m}\right) .
\end{array}
$$

Proof. This follows by the same method as in [15, Theorem 3.3], with Lemma 3.2 therefrom replaced by Lemma 2.25.

The operators $H$ and $G$ are actually independent of $s$ since they are unique extensions by continuity of these operators on the dense subspace $C^{\infty}(X, E)$ of $C\left(C^{\infty}(X, E),|\cdot|_{-s-2 m}\right)$.

When restricted to $L^{2}(X, E)$, the operator $G$ is selfadjoint. Indeed, given any $F, v \in L^{2}(X, E)$, we may invoke the elliptic regularity of the Dirichlet problem (2.65) to conclude that both $G F$ and $G v$ belong to $H^{2 m}(X, E)$ and satisfy the boundary condition $t(\cdot)=0$. It follows that $L G F=\Delta G F$ and $L G v=\Delta G v$ whence

$$
(G F, v)=(G F, H v+L G v)=\left(G F, A^{*} A G v\right)=\left(A^{*} A G F, G v\right)=(F, G v)
$$

which is due to Theorem 2.26. Hence the Schwartz kernel of $G$ is Hermitean, that is, $K_{G}(x, y)^{*}=K_{G}(y, x)$ for all $(x, y)$ away from the diagonal of $X \times X$.

COROLLARY 2.27. If, in addition, $F \in H^{-s-2 m}(X, E)$, then there is a sequence of sections $u_{v} \in C^{\infty}(X, E)$ with $t\left(u_{v}\right)=0$, such that

$$
\left\|u_{v}-u\right\|_{-s} \longrightarrow 0, \quad\left\|\Delta u_{v}-F\right\|_{-s-2 m} \rightarrow 0
$$

as $v \rightarrow \infty$. 
From Lemma 2.24, we deduce that when $u$ is smooth enough, it fulfills $t(u)=0$ if and only if $(\Delta u, v)=(u, \Delta v)$ for all $v$ satisfying $t(v)=0$. This gives rise to the concept of a weak extension of $\Delta$ under the boundary conditions $t(u)=0$ (cf. [5, Chapter 2]). Given an $F \in C\left(C^{\infty}(X, E),|\cdot|_{-s-2 m}\right)$, a section $u$ is said to be a weak solution of (2.65) if it is in $H^{-s^{\prime}}(X, E)$ for some $s^{\prime} \geq 0$ and

$$
(u, \Delta v)=(F, v)
$$

for all $v \in C^{\infty}(X, E)$ satisfying $t(v)=0$.

LEMmA 2.28. Suppose that $F \in C\left(C^{\infty}(X, E),|\cdot|_{-s-2 m}\right)$ where $s \geq 0$. If $u \in$ $H^{-s^{\prime}}(X, E)$ is a weak solution of (2.65), then actually $u \in H^{-s}(X, E)$ and it is a strong solution of (2.65). Moreover, there is a constant $c$ not depending on $F$ or $u$, such that

$$
\|u\|_{-s} \leq c\left(|F|_{-s-2 m}+\|u\|_{-s^{\prime}}\right) .
$$

Proof. See [14].

To study the Dirichlet problem with nonzero boundary data $t(u)=u_{0}$, we need a result of [13]. Denote by $H^{-s, B}(X, E)$ the completion of $C^{\infty}(X, E)$ with respect to the norm

$$
\|u\|_{-s, B}:=\|u\|_{-s}+\|t(u)\|_{\oplus H^{-s-m_{j}-1 / 2}\left(\partial X, F_{j}\right)}+\|n(A u)\|_{\oplus H^{-s-2 m+m_{j}+1 / 2}\left(\partial X, F_{j}\right)} .
$$

The advantage of using these spaces is that for each $u \in H^{-s, B}(X, E)$, there is a sequence $\left\{u_{v}\right\}$ in $C^{\infty}(X, E)$, such that $u_{v} \rightarrow u$ in $H^{-s}(X, E)$, and $\left\{t\left(u_{v}\right)\right\},\left\{n\left(A u_{v}\right)\right\}$ are Cauchy sequences in $\oplus H^{-s-m_{j}-1 / 2}\left(\partial X, F_{j}\right)$ and $\oplus H^{-s-2 m+m_{j}+1 / 2}\left(\partial X, F_{j}\right)$, respectively. Moreover, $\left\{\Delta u_{v}\right\}$ is a Cauchy sequence in $H^{-s-2 m}(X, E)$, which follows by manipulations of Green's formula. Hence to any element $u \in H^{-s, B}(X, E)$, we can assign both $t(u), n(A u)$ and $\Delta u$ defined in the above strong sense.

LEMMA 2.29. For each pair

$$
u_{0} \in \bigoplus_{j=0}^{m-1} H^{-s-m_{j}-1 / 2}\left(\partial X, F_{j}\right), \quad u_{1} \in \bigoplus_{j=0}^{m-1} H^{-s-2 m+m_{j}+1 / 2}\left(\partial X, F_{j}\right),
$$

there is a section $u \in H^{-s, B}(X, E)$ with the property that $t(u)=u_{0}$ and $n(A u)=$ $u_{1}$. Moreover, the mapping $\left(u_{0}, u_{1}\right) \mapsto U$ is continuous in the relevant norms.

Proof. See [13, Lemma 6.1.2].

Given any

$$
F \in C\left(C^{\infty}(X, E),|\cdot|_{-s-2 m}\right), \quad u_{0} \in \oplus H^{-s-m_{j}-1 / 2}\left(\partial X, F_{j}\right),
$$


we now consider the inhomogeneous Dirichlet problem

$$
\Delta u=F \quad \text { in } X, \quad t(u)=u_{0} \quad \text { on } \partial X .
$$

A section $u$ is said to be a weak solution of (2.82) if it is in $H^{-s^{\prime}}(X, E)$ for some $s^{\prime} \geq 0$ and

$$
(u, \Delta v)=(F, v)-\int_{\partial X}\left(u_{0}, n(A v)\right)_{x} d s
$$

for all $v \in C^{\infty}(X, E)$ satisfying $t(v)=0$.

THEOREM 2.30. Suppose that $s \geq 0$. If $F \perp \mathscr{H}(X)$, then there is a weak solution $u \in H^{-s^{\prime}}(X, E)$ to (2.82) with $u \perp \mathscr{H}(X)$. Moreover, $u \in H^{-s}(X, E)$ satisfies (2.82) in a strong sense, and there is a constant $c$ independent of $F, u_{0}$, and $u$, such that

$$
\|u\|_{-s} \leq c\left(|F|_{-s-2 m}+\left\|u_{0}\right\|_{\oplus H^{-s-m_{j}-1 / 2}\left(\partial X, F_{j}\right)}\right) .
$$

Proof. Using Lemma 2.29, we reduce (2.83) to (2.77) with a suitable right side $F$. To this end, we choose $U \in H^{-s, B}(X, E)$ such that $t(U)=u_{0}$ and $n(A U)=$ $u_{1}, u_{1}$ being arbitrary. By the definition of $H^{-s, B}(X, E)$, there is a sequence $\left\{u_{v}\right\}$ in $C^{\infty}(X, E)$ such that

$$
\begin{aligned}
u_{v} & \longrightarrow U \quad \text { in } H^{-s}(X, E), \\
t\left(u_{v}\right) & \longrightarrow u_{0} \quad \text { in } \oplus H^{-s-m_{j}-1 / 2}\left(\partial X, F_{j}\right), \\
n\left(A u_{v}\right) & \longrightarrow u_{1} \quad \text { in } \oplus H^{-s-2 m+m_{j}+1 / 2}\left(\partial X, F_{j}\right),
\end{aligned}
$$

and $\Delta u_{v} \rightarrow F^{\prime}$ in $H^{-s-2 m}(X, E)$.

By Green's formula, we get

$$
\left(u_{v}, \Delta v\right)=\left(\Delta u_{v}, v\right)-\int_{\partial X}\left(t\left(u_{v}\right), n(A v)\right)_{x} d s
$$

for all $v \in C^{\infty}(X, E)$ satisfying $t(v)=0$. Letting $v \rightarrow \infty$ in this equality yields

$$
(U, \Delta v)=\left(F^{\prime}, v\right)-\int_{\partial X}\left(u_{0}, n(A v)\right)_{x} d s .
$$

Subtracting (2.87) from (2.83), we obtain

$$
(u-U, \Delta v)=\left(F-F^{\prime}, v\right)
$$

for all $v \in C^{\infty}(X, E)$ satisfying $t(v)=0$, that is, $u-U$ is a weak solution of the Dirichlet problem (2.65) with $F$ replaced by $F-F^{\prime}$. Moreover, it follows from (2.87) that

$$
\left(F^{\prime}, v\right)=0
$$


for all $v \in \mathscr{H}(X)$. Combining Lemmas 2.28 and 2.25 thus results in the desired assertion.

We now derive a Poisson formula for solutions of the inhomogeneous Dirichlet problem.

To this end, we choose a Green operator $G_{A}(\cdot, \cdot)$ for $A$ on $X$ (cf. [21, Section 9.2.1]). Given an oriented hypersurface $S \subset X$, we denote by $[S]^{A}$ the kernel on $X \times X$ defined by

$$
\left\langle[S]^{A}, g \otimes u\right\rangle_{X \times X}=\int_{S} G_{A}(g, u)
$$

for all $g \in C^{\infty}\left(X, F^{*}\right)$ and $u \in C^{\infty}(X, E)$ whose supports meet each other in a compact set.

In particular, the kernel $[\partial X]^{A}$ is obviously supported on the diagonal of $\partial X \times \partial X$.

For a section $u \in C^{\infty}(X, E)$, we set

$$
(M u)(x)=-G A^{*}\left([\partial X]^{A} u\right)=-\int_{\partial X} G_{A}\left(K_{G A^{*}}(x, \cdot), u\right)
$$

when $x \in \stackrel{\circ}{X}, K_{G A^{*}}$ being the Schwartz kernel of $G A^{*}$. The integral on the righthand side is well defined, for $K_{G A^{*}}$ is a $C^{\infty}$-section of $E \otimes F^{*}$ outside the diagonal of $X \times X$.

COROLlary 2.31. As defined above, $M$ induces a continuous mapping $P$ of $\oplus H^{-s-m_{j}-1 / 2}\left(\partial X, F_{j}\right)$ to $H^{-s}(X, E)$ such that $P t(u)=M u$. Moreover, for each weak solution $u$ of (2.82) it follows that

$$
u=H u+G \Delta u+P t(u) .
$$

Proof. Let $u \in H^{-s^{\prime}}(X, E)$ be a weak solution of (2.82). From Theorem 2.30, we deduce that $u \in H^{-s}(X, E)$ satisfies (2.82) in a strong sense. More precisely, there exists a sequence $u_{v} \in C^{\infty}(X, E)$ which approximates $u$ in $H^{-s}(X, E)$, such that $t\left(u_{v}\right) \rightarrow t(u)$ and $\Delta u_{v} \rightarrow \Delta u$ in the relevant norms. We now set

$$
P u_{0}:=\lim _{v \rightarrow \infty}\left(u_{v}-H u_{v}-G\left(\Delta u_{v}\right)\right)=u-H u-G(\Delta u),
$$

the limit existing in $H^{-s}(X, E)$ by Theorem 2.26. Moreover, it is independent of the particular choice of $u$ with a well-defined $\Delta u$ and $t(u)=u_{0}$, which is again due to Theorem 2.26.

Obviously, $u_{0} \mapsto P u_{0}$ is a continuous mapping of $\oplus H^{-s-m_{j}-1 / 2}\left(\partial X, F_{j}\right)$ to $H^{-s}(X, E)$, and it remains to prove that it agrees with $-G A^{*}\left([\partial X]^{A} u\right)$ in the interior of $X$. 
If $v \in C^{\infty}(X, E)$ has a compact support in the interior of $X$, then by Theorem 2.26 we get

$$
\begin{aligned}
\left(P u_{0}, v\right) & =(u, v)-(u, H v)-(\Delta u, G v) \\
& =(u, v-H v-\Delta(G v))-\int_{\partial X}(t(u), n(A G v))_{x} d s \\
& =-\int_{\partial X}(t(u), n(A G v))_{x} d s
\end{aligned}
$$

for $t(G v)=0$. The right-hand side here just amounts to $\left(-G A^{*}\left([\partial X]^{A} u\right), v\right)$, provided that $u$ is smooth enough.

From (2.93), it follows that $P u_{0}$ is the unique solution of the Dirichlet problem

$$
\Delta u=0 \quad \text { in } X, \quad t(u)=u_{0} \quad \text { on } \partial X,
$$

which is orthogonal to $\mathscr{H}(X)$. We call $P u_{0}$ the Poisson integral of $u_{0}$. By Theorem 2.30,

$$
\left\|P u_{0}\right\|_{-s} \leq c\left\|u_{0}\right\|_{\oplus H^{-s-m_{j}-1 / 2}\left(\partial X, F_{j}\right)}
$$

with $c$ a constant independent of $u_{0}$.

THEOREM 2.32. The quotient space $\mathscr{Y}_{\Delta}^{(f)}(\stackrel{\circ}{X}) / \mathscr{H}(X)$ is topologically isomorphic to $\bigoplus_{j=0}^{m-1} \mathscr{D}^{\prime}\left(\partial X, F_{j}\right)$.

Proof. Given any $u \in \mathscr{Y}_{\Delta}^{(f)}(\stackrel{\circ}{X})$, the Cauchy data $t(u)$ and $n(A u)$, being first defined near $\partial X$, have weak limit values $u_{0}$ and $u_{1}$ on $\partial X$ belonging to $\bigoplus_{j=0}^{m-1} \mathscr{D}^{\prime}\left(\partial X, F_{j}\right)$ (cf. [21, Section 9.4]). Pick a regularisation of $u$ on $\partial X$, that is, any section $U \in H^{-s^{\prime}}(X, E)$ which coincides with $u$ in the interior of $X$ (cf. [21, Section 9.3.6]).

Using the parametrix $G$ of $\Delta$ given by Theorem 2.26, we get by Green's formula

$$
u(x)-H U(x)=-\int_{\partial X}\left(\left(u_{0}, n\left(A K_{G}(\cdot, x)\right)\right)_{y}-\left(u_{1}, t\left(K_{G}(\cdot, x)\right)\right)_{y}\right) d s
$$

for $x \in \stackrel{\circ}{X}$. Since $t\left(K_{G}(\cdot, x)\right)=0$ for all $x \in \stackrel{\circ}{X}$, it follows that $u=H U+P u_{0}$, the section $H U \in \mathscr{H}(X)$ being independent of the particular choice of the regularisation $U$.

We have thus proved that any solution $u \in \mathscr{Y}_{\Delta}^{(f)}(\stackrel{\circ}{X})$ is representable through the weak limit values $t(u)$ on $\partial X$ by the Poisson formula (2.92). Furthermore, $\bigoplus_{j=0}^{m-1} \mathscr{D}^{\prime}\left(\partial X, F_{j}\right)$ is the inductive limit of the sequence $\bigoplus_{j=0}^{m-1} H^{-s-m_{j}-1 / 2} \times$ $\left(\partial X, F_{j}\right), s \in \mathbb{N}$, for the boundary of $X$ is a compact closed $C^{\infty}$-manifold. Combining this with (2.96), we deduce that the mapping $u \mapsto t(u)$ gives the desired isomorphism. 
Since $\mathscr{S}_{A}^{(f)}(\stackrel{\circ}{X})$ is obviously a closed subspace of $\mathscr{Y}_{\Delta}^{(f)}(\stackrel{\circ}{X})$, the mapping

$$
\frac{\mathscr{P}_{A}^{(f)}(\stackrel{\circ}{X})}{\mathscr{H}(X)} \longrightarrow \bigoplus_{j=0}^{m-1} \mathscr{D}^{\prime}\left(\partial X, F_{j}\right)
$$

given by $u \mapsto t(u)$ identifies the quotient space with a closed subspace of $\bigoplus_{j=0}^{m-1} \mathscr{D}^{\prime}\left(\partial X, F_{j}\right)$.

2.5. Hardy spaces. Suppose that $U \Subset \stackrel{\circ}{X}$ is a domain with $C^{\infty}$ boundary. Fix a Dirichlet system $B=\left\{B_{j}\right\}_{j=0}^{m-1}$ of order $m-1$ on $\partial U$, each $B_{j}$ being a differential operator of order $m_{j}$ and type $E \rightarrow F_{j}$ in a neighbourhood $\mathcal{N}$ of $\partial U$. For a section $u$ of $E$ near $\partial U$, we set

$$
t(u)=\left.\bigoplus_{j=0}^{m-1} B_{j} u\right|_{\partial U}
$$

if defined.

Since $\Delta=A^{*} A$ satisfies the condition $(U)_{s}$ in the interior of $X$, the sesquilinear form

$$
h(u, v)=\int_{\partial U}(t(u), t(v))_{x} d s
$$

defines a scalar product on $\mathscr{S}_{\Delta}(U) \cap C^{\infty}(\bar{U}, E)$. Denote by $H_{\Delta}^{(B)}(U)$ the completion of $\mathscr{Y}_{\Delta}(U) \cap C^{\infty}(\bar{U}, E)$ in the norm $u \mapsto \sqrt{h(u, u)}$. These spaces are called the Hardy spaces, by analogy to the classical Hardy spaces of harmonic functions. Alternatively, $H_{\Delta}^{(B)}(U)$ can be described as the space of all $u \in \mathscr{Y}_{\Delta}(U)$ of finite order of growth, for which the weak boundary values of $t(u)$ belong to $\bigoplus_{j=0}^{m-1} L^{2}\left(\partial U, F_{j}\right)$.

LEMMA 2.33. The space $H_{\Delta}^{(B)}(U)$ is a separable Hilbert space with a reproducing kernel.

Proof. By the very definition, $H_{\Delta}^{(B)}(U)$ can be identified as a closed subspace in $\oplus L^{2}\left(\partial U, F_{j}\right)$. In particular, $H_{\Delta}^{(B)}(U)$ is a separable Hilbert space because $\oplus L^{2}\left(\partial U, F_{j}\right)$ is.

Theorem 2.32 implies that each element $u_{0} \in H_{\Delta}^{(B)}(U)$ can be actually thought of as a solution from $\mathscr{Y}_{\Delta}^{(f)}(U)$. To make this more precise, we invoke Theorem 2.30, with $U$ in place of $X$. Since $\mathscr{H}(U)$ is trivial in this case and $L^{2}\left(\partial U, F_{j}\right) \hookrightarrow H^{-m_{j}}\left(\partial U, F_{j}\right)$ for $j=0,1, \ldots, m-1$, there is a unique section $u \in H^{1 / 2}(U, E)$ satisfying $\Delta u=0$ and $t(u)=u_{0}$ in a strong sense. Moreover, we have

$$
\|u\|_{H^{1 / 2}(U, E)} \leq c|| u_{0} \|_{\oplus H^{-m_{j}\left(\partial U, F_{j}\right)}}
$$

with $c$ a constant independent of $u_{0}$. 
By (2.92), we get $u=P_{U} u_{0}$ where $P_{U}$ is the Poisson integral related to the domain $U$. This gives us the desired identification of $H_{\Delta}^{(B)}(U)$ within $\mathscr{Y}_{\Delta}^{(f)}(U)$, for $P_{U}$ is a topological isomorphism of $\bigoplus_{j=0}^{m-1} \mathscr{D}^{\prime}\left(\partial U, F_{j}\right)$ onto $\mathscr{S}_{\Delta}^{(f)}(U)$ (see Theorem 2.32).

Using this fact, we easily conclude that for any $x \in U$ all the evaluation functionals $u \mapsto \delta_{x}^{(j)} u:=u_{j}(x), j=1, \ldots, k$, are continuous on $H_{\Delta}^{(B)}(U)$. Moreover, a stronger property than the continuity holds. Namely, for each compact set $K \subset U$, there is a constant $C_{K}$ such that $\left\|\delta_{x}^{(j)}\right\|<C_{K}$ for all $x \in K$. Hence, $H_{\Delta}^{(B)}(U)$ is a Hilbert space with a reproducing kernel (cf. [1]).

Obviously, $\mathscr{S}_{A}(U) \subset \mathscr{S}_{\Delta}(U)$ holds. Denote by $H_{A}^{(B)}(U)$ the Hardy space for $A$ in $U$, that is, the subspace of $H_{\Delta}^{(B)}(U)$ consisting of all solutions of $A u=0$ in $U$.

LEMMA 2.34. The space $H_{A}^{(B)}(U)$ is a separable Hilbert space with a reproducing kernel.

Proof. The statement follows from Lemma 2.33 once we prove that $H_{A}^{(B)}(U)$ is a closed subspace in $H_{\Delta}^{(B)}(U)$. This latter assertion is a consequence of the Poisson formula (2.92), which shows that the original topology of $H_{\Delta}^{(B)}(U)$ is finer than that induced from $C_{\mathrm{loc}}^{\infty}(U, E)$.

As mentioned, the Dirichlet problem for $\Delta$ in $U$ is uniquely solvable, and its Hodge parametrix $G_{U}$ is, in fact, a Green function of $U$, that is, $H_{U}=0$.

LEMMA 2.35. Let $K(\cdot, \cdot)$ be the reproducing kernel of $H_{\Delta}^{(B)}(U)$. Then, for all $(x, y) \in U \times U$,

$$
K(x, y)=\int_{\partial U}\left(n\left(A K_{G_{U}}(\cdot, x)\right), n\left(A K_{G_{U}}(\cdot, y)\right)\right)_{z} d s
$$

Moreover, for every fixed $x \in U$, the columns of the matrix $K(x, \cdot)$ belong to $\mathscr{Y}_{\Delta}(U) \cap C_{\mathrm{loc}}^{\infty}(\bar{U}, E)$.

Proof. Note that the integral on the right-hand side of (2.102) is well defined for all $(x, y) \in U \times U$, and it belongs to $E_{x}^{*} \otimes \mathscr{Y}_{\Delta}(U)$ in $y$, for every fixed $x \in U$. By the elliptic regularity, it is actually in $E_{x}^{*} \otimes C_{\mathrm{loc}}^{\infty}(\bar{U}, E)$ as claimed.

Let $u \in H_{\Delta}^{(B)}(U)$. Combining the Poisson formula (2.92) and the Bergman formula of Theorem 2.23, we get

$$
0=\int_{\partial U}\left(\left(t(u), n\left(A K_{G_{U}}(\cdot, x)\right)\right)_{z}+(t(u), t(K(x, \cdot)))_{z}\right) d s
$$

for all $x \in U$. Hence it follows that $t(K(x, \cdot))=-n\left(A K_{G_{U}}(\cdot, x)\right)$ on $\partial U$, for each $x \in U$. We thus have

$$
\Delta K(x, \cdot)=0 \quad \text { in } U, \quad t(K(x, \cdot))=-n\left(A K_{G_{U}}(\cdot, x)\right) \quad \text { on } \partial U
$$


for every fixed $x \in U$. Once again, using the Poisson formula, we arrive at (2.102) as desired.

It is obvious that

$$
\begin{aligned}
&\left(\int_{\partial U}(n\right.\left.\left.\left(A K_{G_{U}}(\cdot, x)\right), n\left(A K_{G_{U}}(\cdot, y)\right)\right)_{z} d s\right)^{*} \\
&=\int_{\partial U}\left(n\left(A K_{G_{U}}(\cdot, y)\right), n\left(A K_{G_{U}}(\cdot, x)\right)\right)_{z} d s,
\end{aligned}
$$

which recovers the equality $K(x, y)^{*}=K(y, x)$.

3. Duality for solutions of finite order of growth. In this section, we describe the dual space of $\mathscr{Y}_{A}^{(f)}(U)$ using various pairings in Hilbert spaces of the solutions of the system $A u=0$ in $U$.

3.1. Pairing in Hardy spaces. According to the general scheme, we study in this section and the next one the case

$$
\Sigma_{1}=\mathscr{P}_{\Delta}^{(f)}(U), \quad \Sigma_{2}=\mathscr{Y}_{\Delta}(U) \cap C^{\infty}(\bar{U}, E)
$$

Let $V=H_{\Delta}^{(B)}(U)$. The Poisson formula implies that each element of $H_{\Delta}^{(B)}(U)$ belongs to $\mathscr{S}_{\Delta}^{(f)}(U)$. Write

$$
i_{1}: H_{\Delta}^{(B)}(U) \longrightarrow \mathscr{Y}_{\Delta}^{(f)}(U), \quad i_{2}: \mathscr{Y}_{\Delta}(U) \cap C^{\infty}(\bar{U}, E) \longrightarrow H_{\Delta}^{(B)}(U)
$$

for the canonical embeddings.

The mappings $i_{1}$ and $i_{2}$ are always injective. As mentioned, the Poisson formula (2.92) implies the continuity of $i_{1}$. The mapping $i_{2}$ is continuous because the topology of $\oplus C^{\infty}\left(\partial U, F_{j}\right)$ is stronger than that of $\oplus L^{2}\left(\partial U, F_{j}\right)$. Moreover, it follows from Theorem 2.32 that $\mathscr{S}_{\Delta}(U) \cap C^{\infty}(\bar{U}, E)$ is dense in $\mathscr{S}_{\Delta}^{(f)}(U)$.

The task is now to extend the sesquilinear pairing $h(\cdot, \cdot)$ (see (2.100)) from $H_{\Delta}^{(B)}(U) \times H_{\Delta}^{(B)}(U)$ to $\Sigma_{1} \times \Sigma_{2}$.

Choose a smooth real-valued function $\varrho(x)$ on $X$ with the property that $U=\{x \in X: \varrho(x)<0\}$ and $\nabla \varrho(x) \neq 0$ on $\partial U$. Set

$$
U_{\varepsilon}=\{x \in X: \varrho(x)<\varepsilon\}
$$

then $U_{-\varepsilon} \Subset U \Subset U_{\varepsilon}$ for all sufficiently small $\varepsilon>0$, and $\partial U_{ \pm \varepsilon}$ is as smooth as $\partial U$.

LEMMA 3.1. Given any solutions $u \in \mathscr{S}_{\Delta}^{(f)}(U)$ and $v \in \mathscr{Y}_{\Delta}(U) \cap C^{\infty}(\bar{U}, E)$, the limit

$$
\tilde{h}(u, v)=\lim _{\varepsilon \rightarrow 0+} \int_{\partial U_{-\varepsilon}}(t(u), t(v))_{x} d s_{-\varepsilon}
$$


exists. The corresponding pairing $\tilde{h}: \Sigma_{1} \times \Sigma_{2} \rightarrow \mathbb{C}$ is separately continuous, and its restriction to $H_{\Delta}^{(B)}(U) \times H_{\Delta}^{(B)}(U)$ coincides with $h$.

Proof. We first note that limit (3.4) is none other than the definition of weak boundary values $t(u)$. Therefore, its existence and the separate continuity of the corresponding pairing $\tilde{h}$ is a direct consequence of this definition. The second part of the lemma follows immediately from the definition of Hardy space $H_{\Delta}^{(B)}(U)$.

THEOREM 3.2. The mapping $\mathscr{E}: \mathscr{Y}_{\Delta}(U) \cap C^{\infty}(\bar{U}, E) \rightarrow \mathscr{Y}_{\Delta}^{(f)}(U)^{\prime}$ induced by (3.4) is a topological isomorphism of these spaces.

Proof. Since the natural inclusion $i_{2}$ is one-to-one, the mapping $\mathscr{f}$ is oneto-one, too (see Lemma 2.2).

In this concrete situation, we can easily prove the surjectivity of the mapping directly, that is, without using Theorem 2.3.

Indeed, Theorem 2.32 states that the mapping $t: \mathscr{Y}_{\Delta}^{(f)}(U) \rightarrow \oplus \mathscr{D}^{\prime}\left(\partial U, F_{j}\right)$ is a topological isomorphism, with $t^{-1}$ given by the Poisson integral $P_{U}$ (see (2.92)). Let $\mathscr{F}$ be a continuous linear functional on $\mathscr{Y}_{\Delta}^{(f)}(U)$. Then the composition $\mathscr{F} \circ P_{U}$ is a continuous linear functional on $\oplus \mathscr{D}^{\prime}\left(\partial U, F_{j}\right)$. Since $\oplus \mathscr{D}\left(\partial U, F_{j}^{*}\right)$ is a reflexive space, there is an element $v_{0} \in \oplus \mathscr{D}\left(\partial U, F_{j}^{*}\right)$, such that

$$
\left\langle\mathscr{F} \circ P_{U}, u_{0}\right\rangle=\left\langle u_{0}, v_{0}\right\rangle
$$

for all $u_{0} \in \oplus \mathscr{D}^{\prime}\left(\partial U, F_{j}\right)$.

Set

$$
v=P_{U} *_{\oplus F_{j}}^{-1} v_{0}
$$

then $v \in \mathscr{S}_{\Delta}(U) \cap C^{\infty}(\bar{U}, E)$, which is due to the properties of the Poisson integral. If $u \in \mathscr{Y}_{\Delta}^{(f)}(U)$ then

$$
\begin{aligned}
\mathscr{F}(u) & =\left\langle\mathscr{F}, P_{U} t(u)\right\rangle \\
& =\left\langle\mathscr{F} \circ P_{U}, t(u)\right\rangle \\
& =\left\langle t(u), v_{0}\right\rangle \\
& =\int_{\partial U}(t(u), t(v))_{x} d s \\
& =\tilde{h}(u, v),
\end{aligned}
$$

that is, $\mathscr{F} v=\mathscr{F}$. This proves that $\mathscr{F}$ is surjective.

We have thus proved that the mapping $\mathscr{F}$ is an isomorphism of the vector spaces $\mathscr{Y}_{\Delta}(U) \cap C^{\infty}(\bar{U}, E)$ and $\mathscr{Y}_{\Delta}^{(f)}(U)^{\prime}$. Moreover, both $\mathscr{g}$ and $\mathscr{F}^{-1}$ are continuous, which is clear from the explicit construction of $\mathscr{f}$. This completes the proof. 
COROLlARY 3.3. The mapping $\mathscr{g}^{\prime}: \mathscr{Y}_{\Delta}^{(f)}(U) \rightarrow\left(\mathscr{Y}_{\Delta}(U) \cap C^{\infty}(\bar{U}, E)\right)^{\prime}$, induced by (3.4), is a topological isomorphism of these spaces.

Proof. According to Theorem 2.32, the space $\mathscr{Y}_{\Delta}^{(f)}(U)$ is reflexive, that is, under the natural pairing, we have

$$
\left(\mathscr{Y}_{\Delta}^{(f)}(U)^{\prime}\right)^{\prime} \stackrel{\text { top. }}{\cong} \mathscr{Y}_{\Delta}^{(f)}(U)
$$

where both $\mathscr{Y}_{\Delta}^{(f)}(U)^{\prime}$ and $\left(\mathscr{Y}_{\Delta}^{(f)}(U)^{\prime}\right)^{\prime}$ are endowed with the strong topology of the dual space. Thus, the desired statement follows immediately from Theorem 3.2 .

Since we have a commutative diagram

$$
\begin{aligned}
& \mathscr{Y}_{\Delta}(U) \cap C^{\infty}(\bar{U}, E) \stackrel{i_{2}}{\longrightarrow} H_{\Delta}^{(B)}(U) \stackrel{i_{1}}{\longrightarrow} \mathscr{Y}_{\Delta}^{(f)}(U) \\
& \cup \quad \cup \quad \cup \\
& \mathscr{Y}_{A}(U) \cap C^{\infty}(\bar{U}, E) \stackrel{i_{2}}{\longrightarrow} H_{A}^{(B)}(U) \stackrel{i_{1}}{\longrightarrow} \mathscr{\varphi}_{A}^{(f)}(U),
\end{aligned}
$$

the pairing $\tilde{h}(\cdot, \cdot)$ induces a continuous mapping

$$
\mathfrak{J}: \mathscr{Y}_{A}(U) \cap C^{\infty}(\bar{U}, E) \longrightarrow \mathscr{S}_{A}^{(f)}(U)^{\prime}
$$

which is the restriction of $\mathscr{F}$.

Write $\pi$ for the Szegö projection

$$
\pi: H_{\Delta}^{(B)}(U) \longrightarrow H_{A}^{(B)}(U)
$$

COROLLARY 3.4. The mapping $\mathfrak{J}$ is a topological isomorphism of the space $\mathscr{S}_{A}(U) \cap C^{\infty}(\bar{U}, E)$ onto $\mathscr{Y}_{A}^{(f)}(U)^{\prime}$ if and only if

(1) $\mathscr{S}_{A}(U) \cap C^{\infty}(\bar{U}, E)$ is dense in $\mathscr{Y}_{A}^{(f)}(U)$;

(2) $\pi$ maps $\mathscr{S}_{\Delta}(U) \cap C^{\infty}(\bar{U}, E)$ continuously into $\mathscr{S}_{A}(U) \cap C^{\infty}(\bar{U}, E)$.

Proof. Set

$$
\Im_{1}=\mathscr{Y}_{A}^{(f)}(U), \quad \Im_{2}=\mathscr{Y}_{A}(U) \cap C^{\infty}(\bar{U}, E), \quad \mathfrak{V}=H_{A}^{(B)}(U)
$$

and apply Corollary 2.7.

EXAMPLE 3.5. Let $X=\mathbb{R}^{n}, n \geq 3$, and let $U$ be a ball. Consider a Dirac operator $A$ in $\mathbb{R}^{n}$, that is, a homogeneous first-order differential operator with constant coefficients, such that $-A^{*} A$ is a diagonal matrix with the usual Laplace operator on the diagonal. 
It is proved in [16] that there are systems $\left\{b_{\nu}\right\}$ and $\left\{c_{\mu}\right\}$ of ( $\mathbb{C}^{k}$-valued) homogeneous harmonic polynomials, such that

(a) $\left\{b_{v}\right\}$ is an orthogonal basis in all the spaces $\mathscr{S}_{A}^{(s)}(U), s \in \mathbb{Z}_{+}$, simultaneously and an orthonormal basis in $H_{A}^{(B)}(U)$;

(b) $\left\{b_{v}\right\} \cup\left\{c_{\mu}\right\}$ is an orthogonal basis in all the spaces $\mathscr{P}_{\Delta}^{(s)}(U), s \in \mathbb{Z}_{+}$, simultaneously and an orthonormal basis in $H_{\Delta}^{(B)}(U)$.

Property (a) implies, in particular, that condition (1) of Corollary 3.4 is fulfilled. Moreover, the projection $\pi$, if restricted to $\mathscr{S}_{\Delta}^{(s)}(U)$, coincides with the $H^{s}(U, E)$-orthogonal projection $\mathscr{Y}_{\Delta}^{(s)}(U) \rightarrow \mathscr{Y}_{A}^{(s)}(U)$. It follows that $\pi$ maps $\mathscr{Y}_{\Delta}(U) \cap C^{\infty}(\bar{U}, E)$ continuously into $\mathscr{Y}_{A}(U) \cap C^{\infty}(\bar{U}, E)$. The isomorphism of Corollary 3.4 holds for $A$.

EXAMPLE 3.6. Let $A$ be a determined elliptic operator such that both $A$ and $A^{*}$ possess the unique continuation property. Then condition (1) of Corollary 3.4 holds true.

Write $H_{A^{*}}^{(C)}(U)$ for the closed subspace of the Hardy space $H_{A A^{*}}^{(C)}(U)$ consisting of all solutions to $A^{*} g=0$ in $U$. We consider the extension of $A$ to an operator

$$
T: H_{\Delta}^{(B)}(U) \longrightarrow H_{A^{*}}^{(C)}(U),
$$

whose domain $\mathscr{D}_{T}$ consists of all $u \in H_{\Delta}^{(B)}(U)$ with the property that there is a sequence $\left\{u_{v}\right\}$ in $\mathscr{Y}_{\Delta}(U) \cap C^{\infty}(\bar{U}, E)$, such that

(1) $u_{v} \rightarrow u$ in $H_{\Delta}^{(B)}(U)$;

(2) $A u_{v}$ is a Cauchy sequence in $H_{A^{*}}^{(C)}(U)$.

Using the existence of a two-sided fundamental solution $\Phi$ for $A$, we easily verify that $T$ is a densely defined closed operator.

Let $P_{U}$ denote the Poisson integral of the Dirichlet problem for $A^{*} A$ in $U$. Then the adjoint

$$
T^{*}: \mathscr{D}_{T^{*}} \longrightarrow H_{\Delta}^{(B)}(U)
$$

is given by

$$
T^{*} g=P_{U} n\left(A P_{U} n(g)\right)
$$

for every $g \in \mathscr{Y}_{A^{*}}(U) \cap C^{\infty}(\bar{U}, F)$. Indeed, by the elliptic regularity of the Dirichlet problem, we deduce that $P_{U} n\left(A P_{U} n(g)\right) \in \mathscr{Y}_{\Delta}(U) \cap C^{\infty}(\bar{U}, E)$ for each $g \in$ $\mathscr{Y}_{A^{*}}(U) \cap C^{\infty}(\bar{U}, F)$. Then,

$$
\begin{aligned}
\int_{\partial U}(t(u), & \left.t\left(P_{U} n\left(A P_{U} n(g)\right)\right)\right)_{x} d s \\
= & \int_{\partial U} G_{A}\left(*_{F} A P_{U} n(g), u\right) \\
= & \int_{\partial U} G_{A^{*}}\left(*_{E} P_{U} n(g), A u\right)
\end{aligned}
$$




$$
\begin{aligned}
& =\int_{\partial U}\left(n(A u), t\left(P_{U} n(g)\right)\right)_{x} d s \\
& =\int_{\partial U}(n(A u), n(g))_{x} d s
\end{aligned}
$$

for all $u \in \mathscr{D}_{T}$. In particular, this means that $T^{*}$ is a closed densely defined operator.

Further, the existence of a fundamental solution implies that the range of $T$ is closed and equals $H_{A^{*}}^{(C)}(U)$. Hence $T^{*}$ and $L^{1}=T T^{*}$ are one-to-one operators with closed range.

It is clear that $T T^{*}=\left(A P_{U} n\right)^{2}$. To identify the operator $N^{1}=\left(T T^{*}\right)^{-1}$, we denote by $P_{U}^{1}$ the Poisson integral of the Dirichlet problem for $A A^{*}$ in $U$. Then we have

$$
\left(A P_{U} n\right)^{-1} g=P_{U}^{1} t\left(\Phi_{U} g\right)
$$

for all $g \in H_{A^{*}}^{(C)}(U)$. Indeed, by the properties of the Poisson integral, we see that

$$
n\left(P_{U}^{1} t\left(\Phi_{U} \mathcal{g}\right)\right)=t\left(\Phi_{U} \mathcal{g}\right), \quad P_{U} t\left(\Phi_{U} \mathcal{g}\right)=\Phi_{U} \mathcal{g}, \quad A P_{U} t\left(\Phi_{U} \mathcal{g}\right)=\mathfrak{g}
$$

because $A^{*} A \Phi_{U} \mathcal{g}=A^{*} \mathcal{g}=0$ in $U$.

Finally, since $\Phi$ has the transmission property with respect to $\partial U$ and the Dirichlet problem is elliptic, we conclude that $\left(A P_{U} n\right)^{-1}$ maps $C^{\infty}(\bar{U}, F)$ to itself. Therefore, the projection $\pi$ maps $\mathscr{S}_{\Delta}(U) \cap C^{\infty}(\bar{U}, E)$ continuously into $\mathscr{S}_{A}(U) \cap C^{\infty}(\bar{U}, E)$ (see Section 2.3). By Corollary 3.4, 3 is a topological isomorphism of

$$
\mathscr{Y}_{A}(U) \cap C^{\infty}(\bar{U}, E) \stackrel{\cong}{\longrightarrow} \mathscr{Y}_{A}^{(f)}(U)^{\prime} .
$$

EXAMPLE 3.7. Suppose that $A$ is included into an elliptic compatibility complex of differential operators $A^{i} \in \operatorname{Diff}^{m_{i}}\left(X ; E^{i}, E^{i+1}\right), i=0,1, \ldots, N$, over $X$, with $A^{0}=A$. As usual, we introduce the Laplace operators $\Delta^{i}=A^{i *} A^{i}+$ $A^{i-1} A^{i-1 *}$ for every $i$. They are not elliptic in general, for the orders $m_{i}$ may be pairwise different.

However, any $\Delta^{i}$ admit a well-posed Dirichlet problem (see Section 2.4). Namely, denote by $B^{i}$ the boundary system consisting of the Cauchy data with respect to $A^{i}$ and the Cauchy data with respect to $A^{i-1 *}$ on $\partial U$. In the notation of [22], these are $t(u)$ and $n(u)$, respectively. It is easy to verify that if $\Delta^{i} u=0$ in $U$ and $t(u)=0, n(u)=0$ on $\partial U$, then $u$ actually satisfies $A^{i} u=0$ and $A^{i-1 *} u=0$ in $U$. Since the complex is elliptic, we deduce that $u$ is a $C^{\infty}$ section of $E^{i}$ with a support in $\bar{U}$.

Suppose that any Laplacian $\Delta^{i}$ has the property $(U)_{s}$ in $\stackrel{\circ}{X}$. Then we can introduce Hardy spaces $H_{\Delta^{i}}^{\left(B^{i}\right)}(U)$ as in Section 2.5. Since $A \Delta=\Delta A$, the differential 
A preserves the elements of $H_{\Delta^{i}}^{\left(B^{i}\right)}(U)$ that are sufficiently smooth up to the boundary. Hence $\left\{E^{i}, A^{i}\right\}$ gives rise to a complex of closed operators in Hilbert spaces

$$
0 \longrightarrow H_{\Delta}^{(B)}(U) \stackrel{T}{\longrightarrow} H_{\Delta^{1}}^{\left(B^{1}\right)}(U) \stackrel{T}{\longrightarrow} \cdots \stackrel{T}{\longrightarrow} H_{\Delta^{N}}^{\left(B^{N}\right)}(U) \longrightarrow 0
$$

(see Section 2.3).

The complex (3.21) has a distinguished subcomplex corresponding to the subspaces of $H_{\Delta^{i}}^{\left(B^{i}\right)}(U)$ with $t(u)=0$. Factorising (3.21) by this subcomplex leads to the so-called tangential complex on $\partial U$. More precisely, the spaces of the tangential complex are those subspaces of $H_{\Delta^{i}}^{\left(B^{i}\right)}(U)$ which consist of sections with $n(u)=0$, while its differential $T_{b}$ is a quotient of $T$. By the unique solvability of the Dirichlet problem for $\Delta^{i}$ in $U$, these latter subspaces can be specified as spaces of $L^{2}$-sections of certain vector bundles $E_{b}^{i}$ over the boundary of $U$. In particular, $E_{b}^{0}=\left.\bigoplus_{j=0}^{m-1} F_{j}\right|_{\partial U}$. In fact, the tangential complex corresponds to a complex $\left\{E_{b}^{i}, A_{b}^{i}\right\}$ of differential operators on $\partial U$ (cf. [22, Section 3.1.5]). We get

$$
0 \longrightarrow L^{2}\left(\partial U, E_{b}^{0}\right) \stackrel{T_{b}}{\longrightarrow} L^{2}\left(\partial U, E_{b}^{1}\right) \stackrel{T_{b}}{\longrightarrow} \cdots \stackrel{T_{b}}{\longrightarrow} L^{2}\left(\partial U, E_{b}^{N-1}\right) \longrightarrow 0
$$

so that $H_{A}^{(B)}(U) \cong\left\{u \in L^{2}\left(\partial U, E_{b}^{0}\right): T_{b} u=0\right\}$.

If the domain $U \Subset X$ is strictly pseudoconvex relative to the Levi form of $\left\{E^{i}, A^{i}\right\}$ at step 1 , then the Neumann problem for this complex in $U$ is solvable at step 1. Moreover, the Neumann operator $N$ preserves $C^{\infty}$-sections of $F$ up to the boundary (cf. [22, Section 4.1.5]). Hence the operator $I-T_{b}^{*} N T_{b}$, that is, the orthogonal projection from $L^{2}\left(\partial U, E_{b}^{0}\right)$ to $\operatorname{ker} T_{b}^{0}$ maps $C^{\infty}(\bar{U}, E)$ continuously to itself. As $L^{2}\left(\partial U, E_{b}^{0}\right)=H_{\Delta}^{(B)}(U)$, we see that the projection $\pi$ has the same property.

The question on the density of $\mathscr{S}_{A}(U) \cap C^{\infty}(\bar{U}, E)$ in $\mathscr{S}_{A}^{(f)}(U)$ requires a separate discussion (cf., e.g., [21]). We only mention that this is the case if $A$ is a differential operator with constant coefficients in $\mathbb{R}^{n}$ and $U \Subset \mathbb{R}^{n}$ is convex.

Thus, we can invoke Corollary 3.4 to see that the mapping $\exists$ is a topological isomorphism of $\mathscr{Y}_{A}(U) \cap C^{\infty}(\bar{U}, E)$ onto $\mathscr{S}_{A}^{(f)}(U)^{\prime}$. By reflexivity, the transpose $J^{\prime}$ gives a topological isomorphism of the dual of $\mathscr{S}_{A}(U) \cap C^{\infty}(\bar{U}, E)$ onto $\mathscr{Y}_{A}^{(f)}(U)$.

3.2. Pairing in Lebesgue spaces. As before, we consider

$$
\Sigma_{1}=\mathscr{Y}_{\Delta}^{(f)}(U), \quad \Sigma_{2}=\mathscr{Y}_{\Delta}(U) \cap C^{\infty}(\bar{U}, E) .
$$

Let $V=\mathscr{S}_{\Delta}^{(0)}(U)$. Again, the Poisson formula shows that each element of $\mathscr{Y}_{\Delta}^{(0)}(U)$ belongs to $\mathscr{Y}_{\Delta}^{(f)}(U)$. Write

$$
i_{1}: \mathscr{Y}_{\Delta}^{(0)}(U) \longrightarrow \mathscr{Y}_{\Delta}^{(f)}(U), \quad i_{2}: \mathscr{Y}_{\Delta}(U) \cap C^{\infty}(\bar{U}, E) \longrightarrow \mathscr{Y}_{\Delta}^{(0)}(U)
$$

for the natural inclusions. 
The mappings $i_{1}$ and $i_{2}$ are always injective. As already mentioned, the Poisson formula (2.92) implies the continuity of $i_{1}$. The mapping $i_{2}$ is continuous, too, because the topology of $C^{\infty}(\bar{U}, E)$ is finer than the topology of $L^{2}(U, E)$.

Moreover, it follows from Theorem 2.32 that $\mathscr{S}_{\Delta}(U) \cap C^{\infty}(\bar{U}, E)$ is dense in $\mathscr{Y}_{\Delta}^{(f)}(U)$.

Our next task is to extend the natural sesquilinear pairing $h(\cdot, \cdot)$ from $\mathscr{S}_{\Delta}^{(0)}(U) \times \mathscr{Y}_{\Delta}^{(0)}(U)$ to $\Sigma_{1} \times \Sigma_{2}$.

LEMMA 3.8. For each $u \in \mathscr{Y}_{\Delta}^{(f)}(U)$ and $v \in \mathscr{Y}_{\Delta}(U) \cap C^{\infty}(\bar{U}, E)$, there exists a limit

$$
\tilde{h}(u, v):=\lim _{\varepsilon \rightarrow 0+} \int_{U_{-\varepsilon}}(u, v)_{x} d x
$$

The corresponding pairing $\tilde{h}(\cdot, \cdot)$ is separately continuous on $\Sigma_{1} \times \Sigma_{2}$ and $\tilde{h}(u, v)=h(u, v)$ for all $u, v \in \mathscr{S}_{\Delta}^{(2)}(U)$.

The pairing in formula (3.24) is explicit in [20] in the case of harmonic functions (see [20, pages 573-574]).

Proof. Using Lemma 2.25, we see that there exists a unique $w \in C^{\infty}(\bar{U}, E)$ satisfying

$$
\Delta w=v \quad \text { in } U, \quad t(w)=0 \quad \text { on } \partial U
$$

Then,

$$
\begin{aligned}
\tilde{h}(u, v) & =\lim _{\varepsilon \rightarrow 0+} \int_{U_{-\varepsilon}}(u, v)_{x} d x \\
& =-\lim _{\varepsilon \rightarrow 0+} \int_{\partial U_{-\varepsilon}} G_{\Delta}\left(*_{E} w, u\right) \\
& =\lim _{\varepsilon \rightarrow 0+}\left(\int_{\partial U_{-\varepsilon}}(t(u), n(A w))_{x} d s_{-\varepsilon}-\int_{\partial U_{-\varepsilon}}(n(A u), t(w))_{x} d s_{-\varepsilon}\right) \\
& =\int_{\partial U}(t(u), n(A w))_{x} d s
\end{aligned}
$$

the last equality being due to the existence of weak boundary values $t(u)$ and $n(A u)$ on $\partial U$ (cf. [18]).

The separate continuity of the pairing $\tilde{h}(\cdot, \cdot)$ follows from $(3.27)$ and Lemma 2.25 .

Finally, the restriction of $\tilde{h}(u, v)$ to $u \in \mathscr{Y}_{\Delta}^{(0)}(U)$ and $v \in \mathscr{Y}_{\Delta}(U) \cap C^{\infty}(\bar{U}, E)$ coincides with $h(u, v)$ since the Lebesgue integral is a continuous function of measurable sets.

It is well known that $\mathscr{S}_{\Delta}^{(0)}(U)$ is a separable Hilbert space with a reproducing kernel (see, e.g., [22]). 
As the unique continuation property holds for $\Delta$ in a neighbourhood of $\bar{U}$, the Dirichlet problems for both $\Delta$ and $\Delta^{2}$ in $U$ are uniquely solvable and their Hodge parametrices $G_{U}$ and $G_{\Delta^{2}, U}$ are, in fact, Green functions.

LEMMA 3.9. Let $K(\cdot, \cdot)$ be the reproducing kernel of $\mathscr{Y}_{\Delta}^{(0)}(U)$. Then, for all $(x, y) \in U \times U$,

$$
K(x, y)=-\Delta_{y} \int_{\partial U}\left(n\left(A K_{G_{U}}(\cdot, x)\right), t\left(\Delta K_{G_{\Delta^{2}, U}}(\cdot, y)\right)\right)_{z} d s .
$$

Moreover, for every fixed $x \in U$, the columns of the matrix $K(x, \cdot)$ belong to $\mathscr{S}_{\Delta}(U) \cap C^{\infty}(\bar{U}, E)$.

Proof. Given any fixed $x \in U$, we solve the Dirichlet problem for $\Delta^{2}$ with data

$$
\begin{gathered}
\Delta^{2} V(x, \cdot)=0 \quad \text { in } U, \\
t(V(x, \cdot))=0 \quad \text { on } \partial U, \\
n(A V(x, \cdot))=-n\left(A K_{G_{U}}(\cdot, x)\right) \text { on } \partial U .
\end{gathered}
$$

By the Poisson formula (2.92), for every solution $u \in \mathscr{G}_{\Delta}^{(0)}(U)$ and each $x \in U$, we have

$$
\begin{aligned}
u(x) & =-\int_{\partial U}\left(t(u), n\left(A K_{G_{U}}(\cdot, x)\right)\right)_{y} d s \\
& =\int_{\partial U} G_{\Delta}\left(*_{E} V(x, \cdot), u\right) \\
& =-\int_{U}(u, \Delta V(x, \cdot))_{y} d y
\end{aligned}
$$

whence

$$
0=\int_{U}(u, \Delta V(x, \cdot)+K(x, \cdot))_{y} d y \text {. }
$$

Since the columns of $\Delta V(x, \cdot)+K(x, \cdot)$ belong to $\mathscr{Y}_{\Delta}^{(0)}(U)$, we readily deduce that

$$
\Delta_{y} V(x, y)=-K(x, y)
$$

for all $x, y \in U$. Representing $V(x, y)$ in $y \in U$ by the Poisson formula for $\Delta^{2}$ and using (3.28), we arrive at (3.27).

For each $x, y \in U$, the integral on the right-hand side of (3.27) is well defined, and it belongs to $\mathscr{Y}_{\Delta}(U)$ in $y$ for every fixed $x \in U$. By the elliptic regularity, it is actually in $E_{x}^{*} \otimes\left(\mathscr{S}_{\Delta}(U) \cap C^{\infty}(\bar{U}, E)\right)$ as desired.

THEOREM 3.10. The mapping $\mathscr{E}: \mathscr{\varphi}_{\Delta}(U) \cap C^{\infty}(\bar{U}, E) \rightarrow \mathscr{S}_{\Delta}^{(f)}(U)^{\prime}$ induced by (3.24) is a topological isomorphism of these spaces. 
Proof. Since the natural inclusion $i_{2}$ is injective, the mapping $\mathscr{f}$ is injective too (see Lemma 2.2).

By Theorem 2.3, to prove the surjectivity of the mapping $\mathscr{F}$, we need to show that the reproducing kernel $K(\cdot, \cdot)$ of the space $\mathscr{S}_{\Delta}^{(0)}(U)$ has the following property: for every $\mathscr{F}_{F} \in \mathscr{G}_{\Delta}^{(f)}(U)^{\prime}$, the section $x \mapsto *_{E}^{-1}\langle\mathscr{F}, K(x, \cdot)\rangle$ belongs to the space $\mathscr{Y}_{\Delta}(U) \cap C^{\infty}(\bar{U}, E)$.

Fix any $\mathscr{F}_{\mathcal{F}} \in \mathscr{Y}_{\Delta}^{(f)}(U)^{\prime}$. Since the space $\mathscr{Y}_{\Delta}^{(f)}(U)$ is the inductive limit of the sequence $\mathscr{Y}_{\Delta}^{(-s)}(U), s \in \mathbb{N}$, it can be specified as a subspace of $\mathscr{D}_{\bar{U}}^{\prime}(X, E)$. By the Hahn-Banach theorem, there is a section $v \in C^{\infty}(\bar{U}, E)$ with the property that

$$
\mathscr{F}(u)=\int_{U}(u, v)_{y} d y
$$

for all $u \in \mathscr{Y}_{\Delta}^{(f)}(U)$. In particular, we get

$$
\begin{aligned}
*_{E}^{-1}\langle\mathscr{F}, K(x, \cdot)\rangle & =*_{E}^{-1} \int_{U}(K(x, \cdot), v)_{y} d y \\
& =\int_{U}(v, K(x, \cdot))_{y} d y \\
& =\int_{U}\left(\Delta G_{U} v, K(x, \cdot)\right)_{y} d y \\
& =\int_{\partial U} G_{\Delta}\left(*_{E} K(x, \cdot), G_{U} v\right) \\
& =\int_{\partial U}\left(n\left(A G_{U} v\right), t(K(x, \cdot))\right)_{y} d s
\end{aligned}
$$

if $x \in U$.

Obviously, the integral on the right-hand side of this formula lies in $\mathscr{S}_{\Delta}(U)$. As $v \in C^{\infty}(\bar{U}, E)$, we see that $n\left(A G_{U} v\right)$ belongs to $\bigoplus_{j=0}^{m-1} C^{\infty}\left(\partial U, F_{j}\right)$. From Lemma 3.9 and the regularity properties of the Poisson kernels $K_{G_{U}}(\cdot, \cdot)$ and $K_{G_{\Delta^{2}, U}}(\cdot, \cdot)$, it follows that the section $*_{E}^{-1}\langle\mathscr{F}, K(x, \cdot)\rangle$ is $C^{\infty}$ up to the boundary of $U$ as desired.

Since we have a commutative diagram

$$
\begin{aligned}
& \mathscr{S}_{\Delta}(U) \cap C^{\infty}(\bar{U}, E) \stackrel{i_{2}}{\longrightarrow} \mathscr{Y}_{\Delta}^{(0)}(U) \stackrel{i_{1}}{\longrightarrow} \mathscr{\varphi}_{\Delta}^{(f)}(U) \\
& \cup \quad \cup \quad \cup \\
& \mathscr{S}_{A}(U) \cap C^{\infty}(\bar{U}, E) \stackrel{i_{2}}{\longrightarrow} \mathscr{Y}_{A}^{(0)}(U) \stackrel{i_{1}}{\longrightarrow} \mathscr{\varphi}_{A}^{(f)}(U),
\end{aligned}
$$

the pairing $\tilde{h}(\cdot, \cdot)$ induces a continuous mapping

$$
\mathfrak{J}: \mathscr{Y}_{A}(U) \cap C^{\infty}(\bar{U}, E) \longrightarrow \mathscr{Y}_{A}^{(f)}(U)^{\prime}
$$

which is the restriction of $\mathscr{F}$. 
Write $\pi$ for the Bergman projection

$$
\pi: \mathscr{Y}_{\Delta}^{(0)}(U) \longrightarrow \mathscr{Y}_{A}^{(0)}(U)
$$

COROLLARY 3.11. The mapping $\mathfrak{J}$ is a topological isomorphism of the space $\mathscr{S}_{A}(U) \cap C^{\infty}(\bar{U}, E)$ onto $\mathscr{Y}_{A}^{(f)}(U)^{\prime}$ if and only if

(1) $\mathscr{Y}_{A}(U) \cap C^{\infty}(\bar{U}, E)$ is dense in $\mathscr{S}_{A}^{(f)}(U)$;

(2) $\pi$ maps $\mathscr{Y}_{\Delta}(U) \cap C^{\infty}(\bar{U}, E)$ continuously into $\mathscr{Y}_{A}(U) \cap C^{\infty}(\bar{U}, E)$.

PROof. According to the general scheme, we put

$$
\Im_{1}=\mathscr{Y}_{A}^{(f)}(U), \quad \Im_{2}=\mathscr{Y}_{A}(U) \cap C^{\infty}(\bar{U}, E), \quad \mathfrak{v}=\mathscr{Y}_{A}^{(0)}(U) .
$$

Then the statement follows from Corollary 2.7.

EXAMPLE 3.12. Let $A$ be a determined elliptic operator. In this case, the complex $\left\{E^{i}, A^{i}\right\}$ has only one nonzero operator, which is $A^{0}=A$. It is easy to check that the Neumann problem for this complex (see Section 2.3) is just the Dirichlet problem in $L^{2}(U, F)$ for the Laplacian $A A^{*}$ with the Dirichlet data $n(\cdot)$ instead of $t(\cdot)$.

Suppose that the unique continuation property holds for the formal adjoint $A^{*}$. Then the Neumann operator $N$ is the Green function $G_{A A^{*}, U}$ of the Dirichlet problem.

The elliptic regularity of the Dirichlet problem now implies that $N$ maps $C^{\infty}(\bar{U}, F)$ continuously to $C^{\infty}(\bar{U}, F)$. Therefore, $\pi=I-A^{*} N A$ is a continuous mapping of $C^{\infty}(\bar{U}, E)$ to itself.

Further, by the Runge theorem for determined elliptic operators (cf. [23, Section 4.1.9]), $\mathscr{Y}_{A}(U) \cap C^{\infty}(\bar{U}, E)$ is dense in $\mathscr{Y}_{A}^{(f)}(U)$.

Hence, according to Corollary 3.11, the mapping $J$ is a topological isomorphism of $\mathscr{S}_{A}(U) \cap C^{\infty}(\bar{U}, E)$ onto $\mathscr{S}_{A}^{(f)}(U)^{\prime}$. By reflexivity, the transposed mapping $J^{\prime}$ is a topological isomorphism of the dual to $\mathscr{Y}_{A}(U) \cap C^{\infty}(\bar{U}, E)$ onto $\mathscr{Y}_{A}^{(f)}(U)$.

EXAMPLE 3.13. Let $A$ be a column of scalar differential operators with constant coefficients in $\mathbb{R}^{n}$. Then $A$ can be included into a Hilbert compatibility complex of differential operators with constant coefficients $\left\{E^{i}, A^{i}\right\}$. Under familiar hypotheses on $A$, the compatibility complex is simply a so-called Koszul complex (cf. [22, Section 1.2.8]).

If $U \Subset \mathbb{R}^{n}$ is a strictly convex domain with smooth boundary, then the Neumann problem for $V^{i}=L^{2}\left(U, E^{i}\right)$ is solvable at step 1. Moreover, the Neumann operator $N$ preserves sections from $C^{\infty}(U, F)$ (see ibid).

For any convex domain $U \Subset \mathbb{R}^{n}$, the space $\mathscr{Y}_{A}(U) \cap C^{\infty}(\bar{U}, E)$ is known to be dense in $\mathscr{S}_{A}^{(f)}(U)$. By Corollary 3.11, the mapping $\mathfrak{J}$ is a topological isomorphism of $\mathscr{S}_{A}(U) \cap C^{\infty}(\bar{U}, E)$ onto $\mathscr{Y}_{A}^{(f)}(U)^{\prime}$. The transposed mapping $J^{\prime}$ then gives a topological isomorphism of the dual to $\mathscr{Y}_{A}(U) \cap C^{\infty}(\bar{U}, E)$ onto $\mathscr{S}_{A}^{(f)}(U)$. 
3.3. Grothendieck duality. Suppose that $U$ is an open subset of $\stackrel{\circ}{X}$ with $C^{\infty}$ boundary such that $\partial X \cap \bar{U}=\varnothing$ or $\partial X \cap \bar{U}=\partial X$. Set

$$
\Sigma_{1}=\frac{\mathscr{P}_{\Delta}^{(f)}(U)}{\mathscr{H}(X)}, \quad \Sigma_{2}=\frac{\left\{u \in \mathscr{Y}_{\Delta}(\stackrel{\circ}{X} \backslash \bar{U}) \cap C^{\infty}(X \backslash U, E): t(u)=0 \text { on } \partial X \cap \bar{U}\right\}}{\mathscr{H}(X)}
$$

and

$$
H^{m}((U, \partial X), E)=\left\{u \in H^{m}(U, E): t(u)=0 \text { on } \partial X \cap \bar{U}\right\} .
$$

For $u \in H^{m}(U, E)$, we define

$$
\mathscr{E}_{U}(u) \in \stackrel{\circ}{H}^{m}(X, E)=H^{m}((X, \partial X), E)
$$

by

$$
\begin{gathered}
\mathscr{E}_{U}(u)=u \quad \text { in } U, \\
\Delta \mathscr{E}_{U}(u)=0 \quad \text { in } X \backslash \bar{U}, \\
t\left(\mathscr{E}_{U}(u)\right)=t(u) \quad \text { on } \partial U .
\end{gathered}
$$

Since $A$ possesses the unique continuation property in $\stackrel{\circ}{X}$, the section $\mathscr{E}_{U}(u)$ is uniquely determined for every $u \in H^{m}((U, \partial X), E)$.

Write $H$ for the Hodge projection onto $\mathscr{H}(X)$ (see Theorem 2.26). It was proved in [15] that the Hermitean form

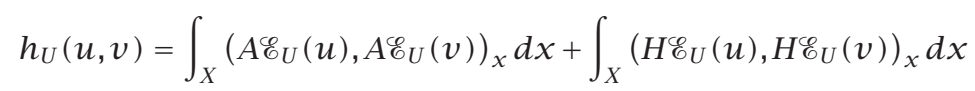

is a scalar product on $H^{m}((U, \partial X), E)$, which induces a topology equivalent to the original one.

Obviously, $\mathscr{H}(X)$ is a closed subspace of

$$
\mathscr{P}_{\Delta}^{(m)}(X \backslash \bar{U}, \partial X):=\mathscr{Y}_{\Delta}(\stackrel{\circ}{X} \backslash \bar{U}) \cap H^{m}((X \backslash \bar{U}, \partial X), E),
$$

and we put

$$
V=\mathscr{Y}_{\Delta}^{(m)}(X \backslash \bar{U}, \partial X) \ominus \mathscr{H}(X),
$$

" $\ominus$ " meaning the orthogonal complement with respect to the scalar product $h_{X \backslash \bar{U}}(\cdot, \cdot)$.

LEMMA 3.14. As defined above, $V$ is a Hilbert space with reproducing kernel. If $\mathscr{H}(X)=0$, then this kernel is given by

$$
K(x, \cdot)=\mathscr{E}_{U} *_{E}^{-1} K_{G}(x, \cdot)
$$

for every $x \in X \backslash \bar{U}$, where $G$ is the Hodge parametrix for the Dirichlet problem in $X$. 
Proof. The space under study is a closed subspace of $\mathscr{Y}_{\Delta}^{(m)}(\stackrel{\circ}{X} \backslash \bar{U})$, hence it is a Hilbert space with reproducing kernel.

If, in addition, $\mathscr{H}(X)=0$, then $G$ is a two-sided fundamental solution of $\Delta$ in the interior of $X$. It follows that $\Delta \mathscr{E}_{U} K_{G}(\cdot, x)=0$ away from $\partial U$ for every fixed $x \in X \backslash \bar{U}$.

By the definition of a reproducing kernel, we get

$$
u(x)=h_{X \backslash \bar{U}}(u, K(x, \cdot))
$$

in $\stackrel{\circ}{X} \backslash \bar{U}$ for all $u \in V$. On the other hand, if $x \in \stackrel{\circ}{X} \backslash \bar{U}$, then, by Stokes' formula, we have

$$
\begin{aligned}
u(x) & =\int_{\partial U} G_{\Delta}\left(K_{G}(x, \cdot), u\right) \\
& =\int_{\partial U}\left(t(u), n\left(A *_{E}^{-1} K_{G}(x, \cdot)\right)\right)_{y} d s-\int_{\partial U}\left(n(A u), t\left(*_{E}^{-1} K_{G}(x, \cdot)\right)\right)_{y} d s \\
& =h_{X \backslash \bar{U}}\left(u, \mathscr{E}_{U} *_{E}^{-1} K_{G}(x, \cdot)\right),
\end{aligned}
$$

which gives the desired conclusion when combined with (3.46).

Define the mapping $i_{2}: \Sigma_{2} \rightarrow V$ in the following way. Pick an element $[u] \in$ $\Sigma_{2}$, $[u]$ being the equivalence class of any $u \in \mathscr{S}_{\Delta}^{(\infty)}(X \backslash \bar{U})$ satisfying $t(u)=0$ on $\partial X \cap \bar{U}$. Obviously, $u \in \mathscr{Y}_{\Delta}^{(m)}(X \backslash \bar{U}, \partial X)$. We set $i_{2}[u]$ to be the orthogonal projection of $u$ to $V$. It is easy to check that $i_{2}: \Sigma_{2} \rightarrow V$ is well defined and continuous.

Further, for $u \in V$, we set $i_{1} u$ to be the equivalence class in $\Sigma_{1}$ of the restriction of $\mathscr{E}_{X \backslash \bar{U}}(u)$ to $U$.

Our next task is to extend the scalar product $h_{X \backslash \bar{U}}(\cdot, \cdot)$ from $V \times V$ to $\Sigma_{1} \times \Sigma_{2}$. Let $[u] \in \Sigma_{1}$ and $[v] \in \Sigma_{2}$. For any representative $u \in[u]$, the Cauchy data $t(u)$ and $n(A u)$ have weak boundary values on the boundary of $U$ (cf. [18]). We set

$$
\tilde{h}_{X \backslash \bar{U}}([u],[v])=-\int_{\partial U} G_{\Delta}\left(*_{E} v, u\right)
$$

where $v \in[v]$.

LEMMA 3.15. Pairing (3.48) does not depend on the particular choice of the representatives $u \in[u]$ and $v \in[v]$. Moreover, it is separately continuous and

$$
\tilde{h}_{X \backslash \bar{U}}\left(i_{1} u,[v]\right)=h_{X \backslash \bar{U}}\left(u, i_{2}[v]\right)
$$

for all $u \in V$ and $[v] \in \Sigma_{2}$. 
Proof. By Stokes' formula, we get

$$
\int_{\partial U} G_{\Delta}\left(*_{E} v, u\right)=\int_{U}(\Delta u, v)_{x} d x-\int_{U}(u, \Delta v)_{x} d x=0
$$

for $v \in \mathscr{H}(X)$. Similarly,

$$
-\int_{\partial U} G_{\Delta}\left(*_{E} v, u\right)=\int_{X \backslash \bar{U}}(\Delta u, v)_{x} d x-\int_{X \backslash \bar{U}}(u, \Delta v)_{x} d x=0
$$

for any $u \in \mathscr{H}(X)$. This shows that $\tilde{h}_{X \backslash \bar{U}}(\cdot, \cdot)$ is independent of the choice of $u \in[u]$ and $v \in[v]$.

By [23, Section 9.4], the convergence of a sequence $\left\{u_{v}\right\}$ in $\mathscr{Y}_{\Delta}^{(f)}(U)$ implies the convergence of both $\left\{t\left(u_{v}\right)\right\}$ and $\left\{n\left(A u_{v}\right)\right\}$ in $\bigoplus_{j=0}^{m-1} \mathscr{D}^{\prime}\left(\partial U, F_{j}\right)$. Therefore, $\tilde{h}_{X \backslash \bar{U}}(\cdot, \cdot)$ is separately continuous provided the spaces $\Sigma_{1}$ and $\Sigma_{2}$ are endowed with the quotient topology.

Since the solutions of $V$ have finite order of growth close to $\partial U$, it follows that

$$
\begin{aligned}
\tilde{h}_{X \backslash \bar{U}}\left(i_{1} u,[v]\right) & =-\int_{\partial U} G_{\Delta}\left(*_{E} v,\left.\mathscr{E}_{X \backslash \bar{U}} u\right|_{U}\right) \\
& =\int_{\partial U}\left(\left(n\left(\left.A \mathscr{E}_{X \backslash \bar{U}} u\right|_{U}\right), t(v)\right)_{y}-\left(t\left(\left.\mathscr{E}_{X \backslash \bar{U}} u\right|_{U}\right), n(A v)\right)_{y}\right) d s \\
& =\int_{\partial U}\left(n\left(\left.A \mathscr{E}_{X \backslash \bar{U}} u\right|_{U}\right), t\left(\mathscr{E}_{X \backslash \bar{U}} v\right)\right)_{y} d s-\int_{\partial U}(t(u), v(A v))_{y} d s \\
& =h_{X \backslash \bar{U}}(u, v)-\int_{X}\left(H^{\mathscr{E}} \mathscr{E}_{X \backslash \bar{U}} u, H^{\mathscr{E}_{X \backslash \bar{U}}} v\right)_{X} d x \\
& =h_{X \backslash \bar{U}}(u, v),
\end{aligned}
$$

the last equality being a consequence of the fact that $w$ is orthogonal to $\mathscr{H}(X)$ with respect to $h_{X \backslash \bar{U}}(\cdot, \cdot)$. The right-hand side here is obviously equal to $h_{X \backslash \bar{U}}\left(u, i_{2}[v]\right)$, which completes the proof.

This lemma gives rise to a mapping $\mathscr{F}: \Sigma_{2} \rightarrow \Sigma_{1}^{\prime}$ induced by the pairing $h_{X \backslash \bar{U}}(\cdot, \cdot)$.

THEOREM 3.16. The mapping

$$
\mathscr{F}: \frac{\mathscr{S}_{\Delta}^{(\infty)}(\stackrel{\circ}{X} \backslash \bar{U}, \partial X)}{\mathscr{H}(X)} \longrightarrow\left(\frac{\mathscr{Y}_{\Delta}^{(f)}(U)}{\mathscr{H}(X)}\right)^{\prime}
$$

induced by (3.48) is a topological isomorphism of these spaces. 
Proof. By the very construction, the mapping $i_{2}$ is one-to-one. Lemma 2.2 shows that $\mathscr{F}$ is one-to-one, too.

We prove the surjectivity of $\mathscr{F}$. To this end, pick a continuous linear functional $\mathscr{F}$ on $\Sigma_{1}$. Since $\mathscr{H}(X)$ is finite dimensional, $\mathscr{F}$ can be specified to a continuous linear functional on $\mathscr{S}_{\Delta}^{(f)}(U)$ vanishing on $\mathscr{H}(X)$. By Theorem 3.10, there is a section $w \in \mathscr{Y}_{\Delta}(U) \cap C^{\infty}(\bar{U}, E)$ orthogonal to $\mathscr{H}(X)$, with the property that

$$
\langle\mathscr{F},[u]\rangle=\int_{U}(u, w)_{x} d x
$$

for all $[u] \in \Sigma_{2}, u$ being a representative of $[u]$.

Set

$$
v(y)=*_{E}^{-1} \int_{U}\left(K_{G}(\cdot, y), w\right)_{x} d x=G\left(x_{U} w\right)(y)
$$

for $y \in \stackrel{\circ}{X}$, where $\chi_{U}$ is the characteristic function of $U$. By Theorem 2.26, we deduce that $v \in H^{2 m}(X, E)$.

Further, $G$ is a Hodge parametrix whence $t(v)=0$ on $\partial X$. We assert that $\Delta v=0$ away from $\bar{U}$ in the interior of $X$.

Indeed, let $u \in C^{\infty}(X, E)$ have compact support in $\stackrel{\circ}{X} \backslash \bar{U}$. Then

$$
\begin{aligned}
\int_{X \backslash \bar{U}}(\Delta u, v)_{y} d y & =\int_{X}(\Delta u, v)_{y} d y \\
& =\int_{U}(G \Delta u, w)_{x} d x \\
& =\int_{U}(u, w)_{x} d x-\int_{U}(H u, w)_{x} d x \\
& =0
\end{aligned}
$$

for $u \equiv 0$ in $U$ and $w$ is orthogonal to $\mathscr{H}(X)$.

Since $G$ has the transmission property with respect to $\partial U$, we see that $v \in$ $C^{\infty}(\bar{U}, E)$ whence $t(v) \in \bigoplus_{j=0}^{m-1} C^{\infty}\left(\partial U, F_{j}\right)$. The elliptic regularity of the Dirichlet problem now yields $v \in C^{\infty}(X \backslash U, E)$, and so $u$ determines an equivalence class $[v] \in \Sigma_{2}$.

If $u \in \mathscr{S}_{\Delta}^{(f)}(U)$, then

$$
u(x)=-\lim _{\varepsilon \rightarrow 0+} \int_{\partial U_{-\varepsilon}} G_{\Delta}\left(K_{G}(x, \cdot), u\right)+\lim _{\varepsilon \rightarrow 0+} H\left(x_{U_{-\varepsilon}} u\right)(x)
$$


for all $x \in U$. Hence it follows that

$$
\begin{aligned}
\langle\mathscr{F},[u]\rangle & =\int_{U}(u, w)_{x} d x \\
& =-\lim _{\varepsilon \rightarrow 0+} \int_{\partial U_{-\varepsilon}} G_{\Delta}\left(*_{E} v, u\right)+\lim _{\varepsilon \rightarrow 0+} \int_{U}\left(H\left(x_{U_{-\varepsilon}} u\right), w\right)_{x} d x \\
& =-\lim _{\varepsilon \rightarrow 0+} \int_{\partial U_{-\varepsilon}} G_{\Delta}\left(*_{E} v, u\right) \\
& =\tilde{h}_{X \backslash \bar{U}}(u, v)
\end{aligned}
$$

because $w$ is orthogonal to $\mathscr{H}(X)$.

Finally, the topological arguments are the same as in Theorem 3.2, which completes the proof.

3.4. Pairing in Sobolev spaces. Set

$$
\Sigma_{1}=\frac{\mathscr{S}_{\Delta}^{(f)}(U)}{\mathscr{H}(X)}, \quad \Sigma_{2}=\frac{\mathscr{P}_{\Delta}(U) \cap C^{\infty}(\bar{U}, E)}{\mathscr{H}(X)}, \quad V=\mathscr{Y}_{\Delta}^{(m)}(U) \ominus \mathscr{H}(X),
$$

" $\ominus$ " meaning the orthogonal complement with respect to the scalar product $h_{U}(\cdot, \cdot)$. When equipped with the scalar product $h_{U}(\cdot, \cdot)$, the space $V$ is obviously Hilbert.

For $[u] \in \Sigma_{2}$, we set

$$
E_{U}([u])=\left[\left.\mathscr{E}_{U}(u)\right|_{X \backslash \bar{U}}\right]
$$

the right-hand side being an equivalence class in $\mathscr{P}_{\Delta}^{(\infty)}(X \backslash \bar{U}, \partial X) / \mathscr{H}(X)$. This class is independent of which representative $u$ of $[u]$ we choose to define it. Indeed, from the unique continuation property for $\Delta$ and the elliptic regularity of the Dirichlet problem, it follows that $\mathscr{E}_{U}$ induces a topological isomorphism of $\mathscr{Y}_{\Delta}(U) \cap C^{\infty}(\bar{U}, E)$ onto $\mathscr{S}_{\Delta}^{(\infty)}(X \backslash \bar{U}, \partial X)$. In particular, if $u \in \mathscr{H}(X)$, then $\mathscr{E}_{U}(u)=u$ in the complement of $\bar{U}$. This gives us the desired independence, hence $E_{U}$ is well defined.

Since the space $\mathscr{H}(X)$ is finite dimensional, we immediately obtain the following lemma.

LEMMA 3.17. The mapping $E_{U}$ is a topological isomorphism

$$
\frac{\mathscr{S}_{\Delta}(U) \cap C^{\infty}(\bar{U}, E)}{\mathscr{H}(X)} \stackrel{\cong}{\cong} \frac{\mathscr{P}_{\Delta}^{(\infty)}(X \backslash \bar{U}, \partial X)}{\mathscr{H}(X)} .
$$

Combining Lemma 3.17 and Theorem 3.16, we see that $\Sigma_{2}$ and $\Sigma_{1}^{\prime}$ are topologically isomorphic. However, we want to recover this result within the general scheme of Section 2. 
To this end, we write $i_{1}: V \rightarrow \Sigma_{1}$ and $i_{2}: \Sigma_{2} \rightarrow V$ for the natural embeddings. They are obviously injective and continuous. We define an extension $\tilde{h}_{U}(\cdot, \cdot)$ of $h_{U}(\cdot, \cdot)$ by

$$
\tilde{h}_{U}([u],[v])=\tilde{h}_{X \backslash \bar{U}}\left([u], E_{U}([v])\right)
$$

LEMmA 3.18. As defined by (3.62), the pairing $\tilde{h}_{U}(\cdot, \cdot)$ does not depend on the choice of $u \in[u]$ and $v \in[v]$. Moreover, it is separately continuous and satisfies

$$
\tilde{h}_{U}\left(i_{1} u,[v]\right)=h_{U}\left(u, i_{2}[v]\right)
$$

for all $u \in V$ and $[v] \in \Sigma_{2}$.

Proof. The pairing is independent of the choice of $u \in[u]$ and $v \in[v]$ because so are the pairing $\tilde{h}_{X \backslash \bar{U}}$ and the mapping $E_{U}$.

Furthermore, from the definition of $\mathscr{E}_{U}$ it follows that $\mathscr{E}_{X \backslash \bar{U}} \mathscr{E}_{U}=\mathscr{E}_{U}$ on $\mathscr{S}_{\Delta}^{(m)}(U)$. Hence, by Lemma 3.15, we get

$$
\begin{aligned}
\tilde{h}_{U}\left(i_{1} u,[v]\right) & =\tilde{h}_{X \backslash \bar{U}}\left(i_{1} \mathscr{E}_{X \backslash \bar{U}} \mathscr{E}_{U}(u), E_{U}([v])\right) \\
& =h_{X \backslash \bar{U}}\left(\mathscr{E}_{X \backslash \bar{U}} \mathscr{E}_{U}(u), i_{2} E_{U}([v])\right) \\
& =h_{U}\left(u, i_{2}[v]\right),
\end{aligned}
$$

as desired.

THEOREM 3.19. The mapping

$$
\mathscr{F}: \frac{\mathscr{I}_{\Delta}(U) \cap C^{\infty}(\bar{U}, E)}{\mathscr{H}(X)} \longrightarrow\left(\frac{\mathscr{S}_{\Delta}^{(f)}(U)}{\mathscr{H}(X)}\right)^{\prime}
$$

induced by (3.62) is a topological isomorphism of these spaces.

Proof. As mentioned, this assertion follows from Lemma 3.17 and Theorem 3.16.

As we have a commutative diagram

$$
\begin{aligned}
& \frac{\mathscr{P}_{\Delta}(U) \cap C^{\infty}(\bar{U}, E)}{\mathscr{H}(X)} \stackrel{i_{2}}{\longrightarrow} \mathscr{P}_{\Delta}^{(m)}(U) \ominus \mathscr{H}(X) \stackrel{{ }^{i_{1}}}{\longrightarrow} \frac{\mathscr{P}_{\Delta}^{(f)}(U)}{\mathscr{H}(X)} \\
& \cup \quad \cup \quad \cup \\
& \frac{\mathscr{P}_{A}(U) \cap C^{\infty}(\bar{U}, E)}{\mathscr{H}(X)} \stackrel{i_{2}}{\longrightarrow} \mathscr{P}_{A}^{(m)}(U) \ominus \mathscr{H}(X) \stackrel{{ }^{i_{1}}}{\longrightarrow} \frac{\mathscr{Y}_{A}^{(f)}(U)}{\mathscr{H}(X)},
\end{aligned}
$$


the pairing $\tilde{h}_{U}(\cdot, \cdot)$ induces a continuous mapping

$$
\mathfrak{\jmath}: \frac{\mathscr{P}_{A}(U) \cap C^{\infty}(\bar{U}, E)}{\mathscr{H}(X)} \longrightarrow\left(\frac{\mathscr{S}_{A}^{(f)}(U)}{\mathscr{H}(X)}\right)^{\prime}
$$

which is the restriction of $\mathscr{F}$.

Write $\pi$ for the $h_{U}$-orthogonal projection

$$
\pi: \mathscr{Y}_{\Delta}^{(m)}(U) \ominus \mathscr{H}(X) \longrightarrow \mathscr{Y}_{A}^{(m)}(U) \ominus \mathscr{H}(X) .
$$

COROLLARY 3.20. The mapping $\mathfrak{J}$ is a topological isomorphism of spaces (3.67) if and only if

(1) $\mathscr{Y}_{A}(U) \cap C^{\infty}(\bar{U}, E)$ is dense in $\mathscr{S}_{A}^{(f)}(U)$;

(2) $\pi \operatorname{maps} \mathscr{S}_{\Delta}(U) \cap C^{\infty}(\bar{U}, E)$ continuously into $\mathscr{S}_{A}(U) \cap C^{\infty}(\bar{U}, E)$.

Proof. According to the general scheme, we put

$$
\mathfrak{\Im}_{1}=\frac{\mathscr{P}_{A}^{(f)}(U)}{\mathscr{H}(X)}, \quad \Im_{2}=\frac{\mathscr{Y}_{A}(U) \cap C^{\infty}(\bar{U}, E)}{\mathscr{H}(X)}, \quad \mathfrak{v}=\mathscr{Y}_{A}^{(m)}(U) \ominus \mathscr{H}(X) .
$$

Then the statement follows from Corollary 2.7.

We thus see that Corollary 3.20 is formulated in just the same way as Corollary 3.11 , the only difference being in the pairing $\tilde{h}$ and corresponding orthogonal projection $\pi$.

Note that the projection $\pi$ can be described as the limit of iterations of double layer potentials (cf. $[11,15])$. We next make use of this description in order to find several cases where the conditions (1) and (2) of Corollary 3.20 are fulfilled.

EXAMPLE 3.21. Let $A$ be a determined elliptic homogeneous operator of order $m<n / 2$ with constant coefficients in $X=\mathbb{R}^{n}$. Then the Laplacian $\Delta=$ $A^{*} A$ has a two-sided fundamental solution of convolution type vanishing at infinity (see, e.g., [21]). By the Liouville theorem, we deduce that $\mathscr{H}(X)=\{0\}$.

Since $\Delta$ is formally selfadjoint, there is a formally selfadjoint fundamental solution of convolution type, say $G$. It was proved in [15] that

$$
\pi=\lim _{N \rightarrow \infty} M^{N}
$$

where

$$
(M u)(x)=-\int_{\partial U} G_{A}\left(K_{G A^{*}}(x-\cdot), u\right)
$$

for all $u \in H^{m}(U, E)$, the limit being in the strong operator topology of $H^{m}(U, E)$.

We can certainly assume that $G$ is of the form $G=\operatorname{ad} A(D) \operatorname{ad} A^{*}(D) g$ where $\operatorname{ad} A(D)$ is the inverse array of $A(D)$ and $g$ is a fundamental solution of the 
(scalar) operator $\operatorname{det} \Delta(D)=|\operatorname{det} A(D)|^{2}$. It is easy to verify that in this particular case the kernel $K_{G A^{*}}(x-y)$ gives a two-sided fundamental solution for $A$. Hence $M^{2}=M$ and $\pi=M$.

Finally, as $G A^{*}$ has the transmission property with respect to any smooth hypersurface in $\mathbb{R}^{n}$, we conclude that $M$ maps $C^{\infty}(\bar{U}, E)$ continuously into $\mathscr{Y}_{A}(U) \cap C^{\infty}(\bar{U}, E)$.

EXAMPLE 3.22. Let $A$ be a determined elliptic operator as in Example 3.12, and let $A^{*}$ possess the unique continuation property in the interior of $X$. Then the condition (1) of Corollary 3.20 is fulfilled.

We are going to invoke the Neumann operator for the short complex consisting of $A_{0}=A$ only (see Section 2.3 in order to prove that the condition (2) of Corollary 3.20 also holds).

Consider the continuous operator $T: H^{m}(U, E) \rightarrow L^{2}(U, F)$ induced by $A$, that is, $T u=A u$. According to [15, Theorem 6.2] the adjoint $T^{*}$ of $T$ with respect to $h_{U}(\cdot, \cdot)$ is given by $T_{U} f=G A^{*}\left(\chi_{U} f\right)$ where $A^{*}$ is the formal adjoint of $A$.

As we are interested in the projection to $\mathscr{S}_{A}^{(m)}(U) \ominus \mathscr{H}(X)$, we need to identify the adjoint of the restriction of $T$ to $H^{m}(U, E) \ominus \mathscr{H}(X)$. Obviously, this adjoint is equal to $\left(1-\pi_{\mathscr{H}(X)}\right) T^{*}$ where $\pi_{\mathscr{H}(X)}$ is the orthogonal projection onto $\mathscr{H}(X)$ with respect to $h_{U}(\cdot, \cdot)$.

We have $\pi_{\mathscr{H}(X)}=H_{\mathscr{E}_{U}}$ where $H$ is the $L^{2}(X, E)$-orthogonal projection to $\mathscr{H}(X)$. Since $\Delta\left(T_{U} f\right)=A^{*}\left(\chi_{U} f\right)$ in the sense of distributions in the interior of $X$, we get $\mathscr{E}_{U} T_{U}=T_{U}$ on all of $X$. It follows that

$$
\left(1-\pi_{\mathscr{H}(X)}\right) T^{*}=\left(1-H_{\mathscr{E}}^{\mathscr{E}}\right) T_{U}=(1-H) T_{U} .
$$

The Laplacian $L^{1}=T T^{*}$ is a bounded selfadjoint operator in $L^{2}(U, F)$. Let us show that it is injective.

Indeed, $L^{1} f=0$ if and only if $T_{U} f=0$, for $T^{*}=T_{U}$. Moreover, if $T_{U} f=0$ in $U$, then $T_{U} f=E_{U} T_{U} f=0$ in $X$, and so $\Delta\left(T_{U} f\right)=A^{*}\left(\chi_{U} f\right)=0$ in $X$. As $A^{*}$ possesses the unique continuation property in a neighbourhood of $U$ we see that $\chi_{U} f \equiv 0$ in $U$.

Since $L^{1}$ is selfadjoint, we conclude that the range of $T T_{U}$ is dense in $L^{2}(U, F)$. Moreover, the range of $T$ is equal to $L^{2}(U, F)$, and so the range of $T_{U}=T^{*}$ is closed in $H^{m}(U, E)$, too. It follows that $H^{m}(U, E)=\operatorname{ker} T \oplus T_{U} L^{2}(U, F)$, hence the range of $L^{1}$ coincides with that of $T$. We have thus proved that $L^{1}$ is an isomorphism of $L^{2}(U, F)$. In fact, $L^{1}=1$ in the case considered in Example 3.21.

We next show that $T_{U}\left(L^{1}\right)^{-1} T$ maps $C^{\infty}(\bar{U}, E)$ continuously to $C^{\infty}(\bar{U}, E)$, and hence $\pi$ does so. For this purpose, pick $g \in L^{2}(U, F)$. Then there exists $f \in$ $L^{2}(U, F)$ satisfying $T T_{U} f=g$ in $U$.

Note that $A T_{U} f$ is defined on all of $X$ and belongs to $L^{2}(X, F)$. Therefore, $g$ can be extended to a section $\tilde{g} \in L^{2}(X, F)$ in such a way that $A T_{U} f=\tilde{g}$ in $X$. It 
follows from Theorem 2.26 that

$$
A^{*} \tilde{g}=A^{*} A T_{U} f=(1-H) A^{*}\left(\chi_{U} f\right)=A^{*}\left(\chi_{U} f\right)
$$

in $H^{-m}(X, E)$, for $H A^{*}=0$.

This formula implies, in particular, that $A A^{*} \tilde{g}=0$ away from $\bar{U}$ in the interior of $X$, and thus $\tilde{g}$ is $C^{\infty}$ on this set.

Since $A^{*}$ (as well as $A$ ) possesses the unique continuation property in $\stackrel{\circ}{X}$, there exists a two-sided fundamental solution to this operator, say $\Phi_{A^{*}}$ in a neighbourhood $O$ of $\bar{U}$. Applying it to both sides of formula (3.73) yields for each $x \in U$

$$
\left(L^{1}\right)^{-1} \mathcal{g}(x)=\tilde{g}(x)+\int_{\partial O} G_{A^{*}}\left(K_{\Phi_{A^{*}}}(x, \cdot), \tilde{\mathfrak{g}}\right) .
$$

It is well known that the kernel of $\Phi_{A^{*}}$ is smooth outside of the diagonal $\{x=$ $y$ \}. Therefore, the boundary integral in (3.74) is a $C^{\infty}$-section of $F$ near $\bar{U}$.

We thus deduce that the inverse $\left(L^{1}\right)^{-1}$ preserves the $C^{\infty}$-sections of $F$ over $\bar{U}$.

Finally, if $u \in C^{\infty}(\bar{U}, E)$, then $\left(L^{1}\right)^{-1} A u \in C^{\infty}(\bar{U}, F)$, and so $T_{U}\left(L^{1}\right)^{-1} A u$ belongs to $C^{\infty}(\bar{U}, E)$ because the Green operator $G$ has the transmission property with respect to $\partial U$. The continuity of $\pi$ now follows from the equality $\pi=I-T_{U}\left(L^{1}\right)^{-1} T$ modulo the smoothing operator $H T_{U}\left(L^{1}\right)^{-1} T$. Thus, conditions (1) and (2) of Corollary 3.20 hold for $A$.

EXAMPLE 3.23. Let $A$ be included in an elliptic complex of differential operators $A^{i} \in \operatorname{Diff}^{m}\left(X ; E^{i}, E^{i+1}\right)$ of the same order on $X$ so that $A^{0}=A$. We formulate a particular Neumann problem (see Section 2.3) corresponding to our situation.

We have a continuous operator $A: H^{m}(U, E) \ominus \mathscr{H}(X) \rightarrow L^{2}(U, F)$. Arguing as in Example 3.22, we see that the adjoint $A^{*}$ of $A$ with respect to $h_{U}(\cdot, \cdot)$ is given by $T_{U} f=G A^{*}\left(\chi_{U} f\right)$.

Let $n_{A^{2}}$ represent the Cauchy data with respect the formal adjoint of $A^{2}$ and

$$
H_{n_{A}}^{m}\left(U, E^{2}\right)=\left\{u \in H^{m}\left(U, E^{2}\right): n_{A}(u)=0\right\}
$$

on $\partial U$. Obviously, it is a closed subspace of $H^{m}\left(U, E^{2}\right)$.

For $x \in U$, pick a cutoff function $\omega_{x}$ at $x$, that is, any $C^{\infty}$-function with a compact support in $U$, equal to 1 near $x$ and vanishing outside a larger neighbourhood of $x$. The difference $1-\omega_{x}$ is equal to 1 close to $\partial U$; hence, 
for every $w \in H_{v_{A}}^{m}\left(U, E^{2}\right)$, we get

$$
\begin{aligned}
T_{U}\left(A^{1}\right)^{*} w & =G\left(\omega_{x}+\left(1-\omega_{x}\right)\right) A^{*}\left(\chi_{U} A^{1 *} w\right) \\
& =\left(G \omega_{x}\right) A^{*} A^{1 *} w+\left(G\left(1-\omega_{x}\right)\right) A^{*} A^{1 *}\left(\chi_{U} w\right) \\
& =0
\end{aligned}
$$

at $x$.

Consider the complex

$$
0 \longleftarrow H^{m}(U, E) \stackrel{T_{U}}{\longleftarrow} L^{2}\left(U, E^{1}\right) \stackrel{A^{1 *}}{\longleftarrow} H_{v_{A}}^{m}\left(U, E^{2}\right) \longleftarrow \cdots,
$$

the fragments indicated by dots being unimportant in the sequel.

Under our assumptions on $\left\{E^{i}, A^{i}\right\}$, the Laplacians $\Delta^{i}=A^{i *} A^{i}+A^{i-1} A^{i-1 *}$ are elliptic operators of order $2 m$.

Let $B^{i}$ be the block operator

$$
B^{i}=\left(\begin{array}{c}
A^{i} \\
A^{i-1 *}
\end{array}\right): H^{m}\left(U, E^{i}\right) \longrightarrow \begin{gathered}
L^{2}\left(U, E^{i+1}\right) \\
L^{2}\left(U, E^{i-1}\right)
\end{gathered}
$$

whose Laplacian is $B^{i *} B^{i}=\Delta^{i}$. As $\Delta^{i}$ are elliptic differential operators, the same is true for $B^{i}$.

We endow the space $H^{m}\left(U, E^{2}\right)$ with the scalar product

$$
h_{U}^{2}(f, g)=\int_{X}\left(B^{\mathscr{E} 2}(f), B_{U}^{\mathscr{E}_{U}^{2}}(g)\right)_{x} d x+\int_{X}\left(H^{\mathscr{E}_{U}^{2}}(f), H_{\mathscr{E}_{U}^{2}}^{2}(g)\right)_{x} d x
$$

constructed for $B^{2}$ in the same way as the scalar product $h_{U}=h_{U}^{0}$ on $H^{m}(U, E)$ was constructed for $A$.

Write $G^{2}$ for the Hodge parametrix of the Dirichlet problem corresponding to $\Delta^{2}$ in $X$, and $T_{U}^{(2,2)}$ for the composition $G^{2} A^{1} \chi_{U}$. Then, the adjoint complex to (3.77) is given by

$$
0 \longrightarrow H^{m}(U, E) \stackrel{A}{\longrightarrow} L^{2}\left(U, E^{1}\right) \stackrel{p T_{U}^{(2,2)}}{\longrightarrow} H_{n_{A}}^{m}\left(U, E^{2}\right) \longrightarrow \cdots
$$

where $p: H^{m}\left(U, E^{2}\right) \rightarrow H_{n_{A}}^{m}\left(U, E^{2}\right)$ is the orthogonal projection with respect to $h_{U}^{2}(\cdot, \cdot)$. Indeed, by [15, Theorem 6.2$]$, the adjoint $B^{2 *}$ to $B^{2}$ with respect to $h_{U}^{2}(\cdot, \cdot)$ is given by

$$
T_{U}^{2} f=G^{2} B^{2 *}\left(\chi_{U} f\right)=\left(G^{2} A^{2 *}\left(\chi_{U} f\right), G^{2} A^{1}\left(\chi_{U} f\right)\right)=\left(T_{U}^{(2,1)}, T_{U}^{(2,2)}\right) f,
$$

where $B^{2 *}$ is the formal adjoint of $B^{2}$. 
It follows that $\left(A^{1 *}\right)^{*}=T_{U}^{(2,2)}$ is the adjoint to $A^{1 *}$ in the sense of Hilbert spaces if $A^{1 *}$ is considered as a bounded operator from $H^{m}\left(U, E^{2}\right)$ to $L^{2}\left(U, E^{1}\right)$. Obviously, $p T_{U}^{(2,2)}$ is the adjoint in the sense of Hilbert spaces for the restriction of $A^{1 *}$ to $H_{n_{A}}^{m}\left(U, E^{2}\right)$. Hence the Laplacian of (3.80) at step 1 is the bounded selfadjoint operator

$$
L^{1}=A^{1 *} p T_{U}^{(2,2)}+A T_{U}
$$

on $L^{2}(U, F)$.

From now on, we assume that $\mathscr{H}^{2}(X)=0$. We show that under this assumption the null space of $p T_{U}^{(2,2)}$ just amounts to $\mathscr{Y}_{A^{1}}^{(m)}(U)$. Denote by $G_{U}^{2}$ the Green function of the Dirichlet problem for $\Delta^{2}$ in $U$. Then for every $f \in H^{m}\left(U, E^{2}\right)$, we have

$$
f=M_{U}^{2} f+G_{U}^{2} \Delta^{2} f
$$

by the Poisson formula (2.92). As $\stackrel{\mathscr{E}}{\mathscr{E}}_{U}^{2}(g)=0$ in $X \backslash \bar{U}$ for any $g \in \stackrel{\circ}{H}^{m}\left(U, E^{2}\right)$, we conclude that

$$
\begin{aligned}
h_{U}^{2}\left(M_{U}^{2} f, G_{U}^{2} \Delta^{2} f\right) & =\int_{U}\left(B^{2} M_{U}^{2} f, B^{2} G_{U}^{2} \Delta^{2} f\right)_{x} d x \\
& =\int_{U}\left(\Delta^{2} M_{U}^{2} f, G_{U}^{2} \Delta^{2} f\right)_{x} d x-\int_{\partial U} G_{B^{2 *}}\left(*_{E^{2}} G_{U}^{2} \Delta^{2} f, B^{2} M_{U}^{2} f\right) \\
& =0
\end{aligned}
$$

because $\Delta^{2} M_{U}^{2} f=0$ in $U$ and $G_{U}^{2} \Delta^{2} f \in \stackrel{\circ}{H}^{m}\left(U, E^{2}\right)$. We thus deduce that the Poisson formula gives an orthogonal decomposition with respect to $h_{U}^{2}(\cdot, \cdot)$ if $\mathscr{H}^{2}(X)=0$. Since

$$
\stackrel{\circ}{H}^{m}\left(U, E^{2}\right) \longleftrightarrow H_{n_{A}}^{m}\left(U, E^{2}\right),
$$

we actually see that

$$
\left(H_{n_{A}}^{m}\left(U, E^{2}\right)\right)^{\perp} \longrightarrow\left(\stackrel{\circ}{H^{m}}\left(U, E^{2}\right)\right)^{\perp}=\mathscr{Y}_{\Delta^{2}}^{(m)}(U)
$$

Thus, $p T_{U}^{(2,2)} f=0$ implies $\Delta^{2} T_{U}^{2,2} f=0$ in $U$. On the other hand, in $X$ we have

$$
\Delta^{2} T_{U}^{(2,2)} f=A^{1}\left(\chi_{U} f\right)-H^{2} A^{1}\left(\chi_{U} f\right)=A^{1}\left(\chi_{U} f\right)
$$

because $H^{2} A^{1}=0$. In particular, this means that $A^{1} f=0$ in $U$ if and only if $\Delta^{2} T_{U}^{(2,2)} f=0$ in $U$. 
Conversely, if $A^{1} f=0$ in $U$, then $\Delta^{2} T_{U}^{(2,2)} f=0$ in $U$, and

$$
T_{U}^{(2,2)} f(x)=\int_{\partial U} G_{B^{2 *}}\left(K_{G^{2}}(x, \cdot), 0 \oplus f\right)
$$

for all $x \in U$, the last equality being a consequence of Stokes' formula. Moreover, for all $g \in H_{n_{A}}^{m}\left(U, E^{2}\right)$, we have

$$
\begin{aligned}
h_{U}^{2}\left(T_{U}^{(2,2)} f, g\right) & =\int_{X}\left(B^{2} T_{U}^{(2,2)} f, B^{2 \mathscr{E}_{U}^{2}} g\right)_{x} d x \\
& =\int_{\partial U} G_{B^{2}}\left(0 \oplus *_{E^{1}} f, g\right) \\
& =\int_{\partial U} G_{A^{1 *}}\left(*_{E^{1}} f, g\right) \\
& =0
\end{aligned}
$$

the last equality being due to jump theorems for a single layer potential and the fact that $n_{A}\left(\mathscr{E}_{U}^{2}(g)\right)=n_{A}(g)=0$ on $\partial U$. Hence $A^{1} f=0$ in $U$ implies $p T_{U}^{(2,2)} f=$ 0 in $U$ as desired.

Describe the null space of $L^{1}$. Note that $L^{1} f=0$ if and only if both $T_{U} f$ and $p T_{U}^{(2,2)} f$ vanish, for the operator

$$
\left(\begin{array}{c}
p T_{U}^{(2,2)} \\
T_{U}
\end{array}\right): L^{2}(U, F) \longrightarrow \begin{gathered}
H_{n_{A}}^{m}\left(U, E^{2}\right) \\
H^{m}(U, E)
\end{gathered}
$$

is adjoint to the differential operator $B^{1 *}$.

Hence $\operatorname{ker} L^{1}=\operatorname{ker} T_{U} \cap \mathscr{S}_{A^{1}}^{(0)}(U)$. It was proved in [15] (see Lemma 8.4) that

$$
\operatorname{ker} T_{U} \cap \mathscr{Y}_{A^{1}}(U)=\left\{f \in L^{2}(U, F): A^{*} f=0, A^{1} f=0, n(f)=0 \text { on } \partial U\right\}
$$

This is the so-called harmonic space at step 1 for the differential complex $\left\{E^{i}, A^{i}\right\}$ in $U$. We usually realise it as the null space for the Neumann problem in the $L^{2}$-setting for $\left\{E^{i}, A^{i}\right\}$.

As the Laplacian $L^{1}$ is defined everywhere on $L^{2}(U, F)$, the Neumann problem for complex (3.80) at step 1 reads as follows: given any $f \in L^{2}(U, F)$, find $g \in L^{2}(U, F)$ satisfying $L^{1} g=f$. If the range of $L^{1}$ is closed, then so is the range of $A: H^{m}(U, E) \rightarrow L^{2}(U, F)$. For the Dolbeault complex, this latter fails to be the case even for the small balls $U$ of $\mathbb{C}^{n}$. Hence, for the Dolbeault complex, the Neumann problem in the present setting cannot highlight any properties of the projection $\pi$.

The last observation in Example 3.23 leads us to another choice of function spaces, which could be $H^{m}\left(U, E^{i}\right)$. 
EXAMPLE 3.24. Let $X=\mathbb{R}^{n}, n \geq 3, U \subset \mathbb{R}^{n}$ a ball, and $A$ a Dirac operator in $\mathbb{R}^{n}$ (see Example 3.5).

It is proved in [16] that there are systems $\left\{b_{v}\right\}$ and $\left\{c_{\mu}\right\}$ of ( $\mathbb{C}^{k}$-valued) homogeneous harmonic polynomials such that

(a) $\left\{b_{v}\right\}$ is an orthogonal basis in all the spaces $\mathscr{Y}_{A}^{(s)}(U), s \in \mathbb{Z}_{+}$, simultaneously and an orthonormal basis in $\mathscr{S}_{A}^{(m)}(U)$ with respect to $h_{U}(\cdot, \cdot)$;

(b) $\left\{b_{v}\right\} \cup\left\{c_{\mu}\right\}$ is an orthogonal basis in all the spaces $\mathscr{S}_{\Delta}^{(s)}(U), s \in \mathbb{Z}_{+}$, simultaneously and an orthonormal basis in $\mathscr{G}_{\Delta}^{(m)}(U)$ with respect to $h_{U}(\cdot, \cdot)$.

Property (a) implies, in particular, that condition (1) of Corollary 3.20 is fulfilled. Moreover, the projection $\pi$, if restricted to $\mathscr{Y}_{\Delta}^{(s)}(U)$, coincides with the $h_{U}(\cdot, \cdot)$-orthogonal projection $\mathscr{S}_{\Delta}^{(s)}(U) \rightarrow \mathscr{Y}_{A}^{(s)}(U)$. It follows that $\pi$ maps $\mathscr{Y}_{\Delta}(U) \cap C^{\infty}(\bar{U}, E)$ continuously into $\mathscr{Y}_{A}(U) \cap C^{\infty}(\bar{U}, E)$. Hence Corollary 3.20 holds for $A$.

4. Duality for solutions of arbitrary order of growth. In this section, we describe the dual space of $\mathscr{Y}_{A}(U)$ by using various pairings in Hilbert spaces of the solutions of the system $A u=0$ in $U$. We assume that both $X$ and $A$ are real analytic.

4.1. Duality in Hardy spaces. Let $U \Subset X$ be a domain with real analytic boundary. In this and the next section, we restrict ourselves to the case $\Sigma_{1}=$ $\mathscr{S}_{\Delta}(U)$ and $\Sigma_{2}=\mathscr{Y}_{\Delta}(\bar{U})$. Let $V=H_{\Delta}^{(B)}(U)$ and

$$
i_{1}: H_{\Delta}^{(B)}(U) \longrightarrow \mathscr{Y}_{\Delta}(U), \quad i_{2}: \mathscr{Y}_{\Delta}(\bar{U}) \longrightarrow H_{\Delta}^{(B)}(U)
$$

be natural inclusions.

The mapping $i_{1}$ is always one-to-one, and the mapping $i_{2}$ is one-to-one because of the unique continuation property $(U)_{s}$. As mentioned, the Poisson formula (2.92) implies the continuity of $i_{1}$. The mapping $i_{2}$ is continuous by a priori estimates for solutions of elliptic equations.

From the Runge theorem for the solutions of elliptic systems (see, for instance, [23, Theorem 4.1.26]) it follows that $\mathscr{S}_{\Delta}(\bar{U})$ is dense in $\mathscr{S}_{\Delta}(U)$.

Our next goal is to extend the sesquilinear pairing $h(\cdot, \cdot)$ (see (2.100)) from $H_{\Delta}^{(B)}(U) \times H_{\Delta}^{(B)}(U)$ to $\Sigma_{1} \times \Sigma_{2}$.

Note that the analyticity of $\partial U$ implies that also $\partial D_{ \pm \varepsilon}$ is real analytic for each sufficiently small $\varepsilon>0$.

THEOREM 4.1. Let $0 \leq j \leq m-1$ and let $\delta>0$ be small enough. Then there exist a compact set $K \subset U, \varepsilon_{0}>0$, and $C>0$ depending on $j, \delta, K$, and $\varepsilon_{0}$ such that, for all $u \in \mathscr{S}_{\Delta}(U), v \in \mathscr{Y}_{\Delta}\left(U_{\delta}\right) \cap C^{2 m}\left(\bar{U}_{\delta}, E\right)$, and $\varepsilon \in\left(0, \varepsilon_{0}\right]$,

$$
\left|\int_{\partial U_{-\varepsilon}}\left(B_{j} u, B_{j} v\right)_{x} d s_{-\varepsilon}\right| \leq C\|u\|_{C(K, E)}\|v\|_{C^{2 m}\left(\bar{U}_{\delta}, E\right)}
$$


Proof. Let

$$
n(f)=\bigoplus_{j=0}^{m-1} *_{F_{j}}^{-1} C_{j} *_{F} f
$$

be the Dirichlet system adjoint to the system $t$ with respect to the Green formula for $A$. Given any sufficiently small $\varepsilon>0$, we consider the Cauchy problem

$$
\begin{gathered}
A^{*} A v_{j,-\varepsilon}=0 \text { near } \partial U_{-\varepsilon}, \\
t\left(v_{j,-\varepsilon}\right)=0 \quad \text { on } \partial U_{-\varepsilon}, \\
n\left(A v_{j,-\varepsilon}\right)=\left(0, \ldots, 0, B_{j} v, 0, \ldots, 0\right) \text { on } \partial U_{-\varepsilon}
\end{gathered}
$$

in a neighbourhood of $U_{-\varepsilon}$. The following statement is a consequence of the Cauchy-Kovalevskaya theorem.

LEMMA 4.2. Given any $\delta>0$ small enough, there are $\varepsilon_{0}>0$ and $C_{0}>0$ such that, for all $\varepsilon \in\left(0, \varepsilon_{0}\right]$, the sections $v_{j,-\varepsilon}$ belong to $\mathscr{Y}_{\Delta}\left(U_{-\varepsilon+r} \backslash \bar{U}_{-2 \varepsilon_{0}}\right)$ with some $r>0$ independent of $\varepsilon$ and satisfy

$$
\left\|v_{j,-\varepsilon}\right\|_{C^{2 m}\left(\bar{U}_{-\varepsilon+r} \backslash U_{-2 \varepsilon_{0}}, E\right)} \leq C_{0}\|v\|_{C^{2 m}\left(\bar{U}_{\delta}, E\right)} .
$$

Proof. First, we note that there exists a neighbourhood $O$ of $\partial U$ and sections $W_{j,-\varepsilon}$, real analytic near $\bar{O}$, such that

$$
\begin{gathered}
t\left(W_{j,-\varepsilon}\right)=0 \quad \text { on } \partial U_{-\varepsilon}, \\
n\left(A W_{j,-\varepsilon}\right)=\left(0, \ldots, 0, B_{j} v, 0, \ldots, 0\right) \quad \text { on } \partial U_{-\varepsilon}, \\
\left\|W_{j,-\varepsilon}\right\|_{C^{2 m}(\bar{O}, E)} \leq C_{0}\|v\|_{C^{2 m}\left(\bar{U}_{\delta}, E\right)} .
\end{gathered}
$$

For instance, if $m_{j}=j$, we can take

$$
\begin{aligned}
& W_{0,-\varepsilon}(x)=\frac{(\varrho(x)+\varepsilon)^{2 m-1}}{(2 m-1) !}\left(\sigma^{2 m-1}\left(*_{F_{0}}^{-1} C_{0} *_{F} A\right)(x, \nabla \varrho(x))\right)^{-1} B_{0} v(x), \\
& W_{1,-\varepsilon}(x)=\frac{(\varrho(x)+\varepsilon)^{2 m-2}}{(2 m-2) !}\left(\sigma^{2 m-2}\left(*_{F_{1}}^{-1} C_{1} *_{F} A\right)(x, \nabla \varrho(x))\right)^{-1} B_{1} v(x)-\cdots
\end{aligned}
$$

and so on. 
Hence $v_{j,-\varepsilon}=W_{j,-\varepsilon}+w_{j,-\varepsilon}$ where $w_{j,-\varepsilon}$ is a solution of the Cauchy problem

$$
\begin{gathered}
A^{*} A w_{j,-\varepsilon}=-A^{*} A W_{j,-\varepsilon} \text { near } \partial U_{-\varepsilon} \\
t\left(w_{j,-\varepsilon}\right)=0 \text { on } \partial U_{-\varepsilon} \\
n\left(A w_{j,-\varepsilon}\right)=0 \text { on } \partial U_{-\varepsilon} .
\end{gathered}
$$

As the Dirichlet system $\{t, n \circ A\}$ represents the Cauchy data for the Laplacian $\Delta$, we see that

$$
\begin{gathered}
A^{*} A w_{j,-\varepsilon}=-A^{*} A W_{j,-\varepsilon} \text { near } \partial U_{-\varepsilon} \\
w_{j,-\varepsilon} \text { vanishes up to order } 2 m-1 \text { on } \partial U_{-\varepsilon}
\end{gathered}
$$

Since the hypersurfaces $\partial U_{-\varepsilon}$ are real analytic and compact, we can argue locally. Fix a point $x^{0} \in \partial U$. Then, after a suitable bianalytic change of variables $x=\delta(y)$ in a neighbourhood of $x^{0}$, we obtain the Cauchy problem

$$
\begin{gathered}
\Delta^{\sharp} w_{j,-\varepsilon}(\delta(y))=-A^{*} A W_{j,-\varepsilon}(\delta(y)) \text { if } y_{n}<-\varepsilon, \\
\left(\frac{\partial}{\partial y_{n}}\right)^{k} w_{j,-\varepsilon}(\delta(y)) \text { vanishes for } j=0,1, \ldots, 2 m-1 \text { if } y_{n}=-\varepsilon,
\end{gathered}
$$

where $\Delta^{\sharp}$ is a differential operator of order $2 m$ with real analytic coefficients. Obviously, $\Delta^{\sharp}$ inherits the ellipticity from $\Delta$.

Finally, complexifying problem (4.10) and using (4.6) and [7, Theorem 9.4.5], we arrive at the assertion of the lemma.

Further, let $G_{\Delta}$ be a Green operator for $\Delta$. Then, using Lemma 4.2 and Stokes' formula, we get

$$
\begin{aligned}
\int_{\partial U_{-\varepsilon}} & \left(B_{j} u, B_{j} v\right)_{x} d s_{-\varepsilon} \\
= & \int_{\partial U_{-\varepsilon}}\left(t(u), n\left(A v_{j,-\varepsilon}\right)\right)_{\chi} d s_{-\varepsilon}-\int_{\partial U_{-\varepsilon}}\left(n(A u), t\left(v_{j,-\varepsilon}\right)\right)_{X} d s_{-\varepsilon} \\
= & \int_{\partial U_{-\varepsilon}} G_{\Delta}\left(*_{E} v_{j,-\varepsilon}, u\right) \\
= & \int_{\partial U_{-\varepsilon_{0}}} G_{\Delta}\left(*_{E} v_{j,-\varepsilon}, u\right)
\end{aligned}
$$

for all $0<\varepsilon \leq \varepsilon_{0}$.

Since $G_{\Delta}(\cdot, \cdot)$ is a bidifferential operator of order $2 m-1$ on $\partial U$, we conclude that

$$
\left|\int_{\partial U_{-\varepsilon}}\left(B_{j} u, B_{j} v\right)_{\chi} d s_{-\varepsilon}\right| \leq c\|u\|_{C^{2 m-1}\left(\partial U_{-\varepsilon_{0}}, E\right)} \mid\left\|v_{j,-\varepsilon}\right\|_{C^{2 m-1}\left(\partial U_{-\varepsilon_{0}}, E\right)},
$$


the constant $C$ depending on the coefficients of $A$ and $\left\{B_{j}\right\}$ only. By Lemma 4.2, there is a constant $C_{0}>0$ such that

$$
\left\|v_{j,-\varepsilon}\right\|_{C^{2 m-1}\left(\partial U_{\left.-\varepsilon_{0}, E\right)}\right.} \leq C_{0}\|v\|_{C^{2 m}\left(\bar{U}_{\delta}, E\right)}
$$

for all $v \in \mathscr{Y}_{\Delta}\left(U_{\delta}\right) \cap C^{2 m}\left(\bar{U}_{\delta}, E\right)$. Finally, by a priori estimates for the solutions of elliptic systems, there exists a constant $C_{1}>0$ such that for all $u \in \mathscr{Y}_{\Delta}(U)$ we get

$$
\|u\|_{C^{2 m-1}\left(\partial U_{\left.-\varepsilon_{0}, E\right)}\right.} \leq C_{1}\|u\|_{C\left(\bar{U}_{-\varepsilon_{0} / 2}, E\right)}
$$

Now, combining inequalities (4.12), (4.13), and (4.14), we obtain (4.2) as desired.

In the case where $\Delta$ is the usual Laplace operator in $\mathbb{R}^{n}$, Stout [19] proved that $\partial D$ should be necessarily real analytic for estimate (4.2) to hold.

COROLlary 4.3. For every $u \in \mathscr{S}_{\Delta}(U)$ and $v \in \mathscr{Y}_{\Delta}(\bar{U})$, the limit

$$
\tilde{h}(u, v)=\lim _{\varepsilon \rightarrow 0+} \int_{\partial D_{-\varepsilon}}(t(u), t(v))_{x} d s_{-\varepsilon}
$$

exists. The pairing $\tilde{h}(\cdot, \cdot)$ is separately continuous on $\mathscr{S}_{\Delta}(U) \times \mathscr{S}_{\Delta}(\bar{U})$, and $\tilde{h}(u, v)$ $=h(u, v)$ for all $u \in H_{\Delta}^{(B)}(U)$ and $v \in \mathscr{Y}_{\Delta}(\bar{U})$.

Proof. By the assumption, there exists a $\delta>0$ with the property that $v \in$ $\mathscr{S}_{\Delta}\left(U_{\delta}\right) \cap C^{2 m}\left(\bar{U}_{\delta}, E\right)$.

Given any $\varepsilon \in\left(0, \varepsilon_{0}\right]$, $\varepsilon_{0}$ being from Theorem 4.1 , we define a continuous functional $\mathscr{F}_{v,-\varepsilon}$ on $\mathscr{S}_{\Delta}(U)$ by

$$
\mathscr{F}_{v,-\varepsilon}(u)=\int_{\partial U_{-\varepsilon}}(t(u), t(v))_{x} d s_{-\varepsilon}
$$

for $u \in \mathscr{Y}_{\Delta}(D)$. According to Theorem 4.1, there is a constant $C>0$ independent of $\varepsilon$ such that

$$
\left|\mathscr{F}_{v,-\varepsilon}(u)\right| \leq C\|u\|_{C\left(\bar{U}_{\varepsilon_{0} / 2}, E\right)}
$$

for all $0<\varepsilon \leq \varepsilon_{0}$.

Let $\Sigma$ be the subset of $\mathscr{Y}_{\Delta}(U)$ consisting of all solutions $u$ with the property that

$$
\|u\|_{C\left(\bar{U}_{\varepsilon_{0} / 2}, E\right)}<\frac{1}{C}
$$


This means that for each $\varepsilon \in\left(0, \varepsilon_{0}\right]$ the functional $\mathscr{F}_{v,-\varepsilon}$ belongs to the polar of $\Sigma$, that is,

$$
\Sigma^{\circ}=\left\{\mathscr{F}_{\in} \in \mathscr{T}_{\Delta}(U)^{\prime}:|\mathscr{F}(u)| \leq 1 \forall u \in \Sigma\right\} .
$$

By a familiar theorem of Alaoglu and Banach, this polar is weak* compact. Since the space $\mathscr{S}_{\Delta}(U)$ is separable, this polar is metrisable in the weak* topology. By compactness, there are limit points for the net $\left\{\mathscr{F}_{v,-\varepsilon}\right\}_{0<\varepsilon \leq \varepsilon_{0}}$. Let $\mathscr{F}_{0}$ be such a limit point. Thus, for some sequence $\varepsilon_{k} \in\left(0, \varepsilon_{0}\right]$ converging to 0 , we have

$$
\lim _{k \rightarrow \infty} \mathscr{F}_{v,-\varepsilon_{k}}=\mathscr{F}_{0}(u)
$$

for all $u \in \mathscr{Y}_{\Delta}(U)$.

It is easy to see that for any $u \in H_{\Delta}^{(B)}(U)$ we have

$$
\mathscr{F}_{0}(u)=\lim _{k \rightarrow \infty} \mathscr{F}_{v,-\varepsilon_{k}}(u)=h(u, v) .
$$

Hence, each weak* limit point of the net $\left\{\mathscr{F}_{v,-\varepsilon}\right\}_{0<\varepsilon \leq \varepsilon_{0}}$ agrees on $H_{\Delta}^{(B)}(U)$ with $u \mapsto h(u, v)$.

As $\mathscr{T}_{\Delta}(\bar{U})$ is dense in $\mathscr{S}_{\Delta}(U)$, the space $H_{\Delta}^{(B)}(U)$ is dense there, too. This implies the existence of a limit

$$
\lim _{\varepsilon \rightarrow 0+} \mathscr{F}_{v,-\varepsilon}=\mathscr{F}_{0}
$$

which defines an element of $\mathscr{S}_{\Delta}(U)^{\prime}$.

Finally, the separate continuity of the pairing $\tilde{h}(\cdot, \cdot)$ follows immediately from (4.2).

THEOREM 4.4. The mapping $\mathscr{\mathscr { S }}: \mathscr{Y}_{\Delta}(\bar{U}) \rightarrow \mathscr{Y}_{\Delta}(U)^{\prime}$ induced by (4.15) is a topological isomorphism of these spaces.

Proof. Since the natural inclusion $i_{2}$ is one-to-one, the mapping $\mathscr{F}$ is oneto-one, too (see Lemma 2.2).

According to Corollary 2.5, to prove the surjectivity of the mapping we have to show that the reproducing kernel $K(\cdot, \cdot)$ of the space $H_{\Delta}^{(B)}(U)$ has the following property.

LEMMA 4.5. For every fixed $x \in U$, the Szegö kernel $K(x, \cdot)$ belongs to the space $E_{x}^{*} \otimes \mathscr{Y}_{\Delta}(\bar{U})$.

PROoF. Since $X, \partial D, \Delta$, and $\left\{B_{j}\right\}$ are real analytic, Theorem A of [10] implies that any solution $u$ of the Dirichlet problem (2.82) actually satisfies $\Delta u=0$ in a neighbourhood of $\bar{U}$ if the data $\bigoplus_{j=0}^{m-1} u_{j}$ are real analytic (cf., for instance, 
[12, Lemma 4.4]). This means, in particular, that $G_{U}(x, \cdot)$ is real analytic in a neighbourhood of $\partial U$. Hence, we deduce from (2.104) that $K(x, \cdot) \in E_{x} \otimes \mathscr{Y}_{\Delta}(\bar{U})$ for every fixed $x \in U$ as desired.

We have thus proved that the mapping $\mathscr{F}$ is an isomorphism of vector spaces

$$
\mathscr{S}_{\Delta}(\bar{U}) \stackrel{\cong}{\longrightarrow} \mathscr{T}_{\Delta}(U)^{\prime} .
$$

We are now going to invoke an operator-theoretic argument to conclude that this algebraic isomorphism is in fact a topological one. To this end, we note that the spaces $\mathscr{Y}_{\Delta}(\bar{U})$ and $\mathscr{Y}_{\Delta}(U)^{\prime}$ are both spaces of type $D F S$. For $\mathscr{S}_{\Delta}(\bar{U})$, see the proof of Theorem 1.5.5 in Morimoto [9, page 13]. As the closed graph theorem is correct for linear mappings between spaces of type DFS (see [9, Corollary A.6.4, page 254]), to see that $v \mapsto \mathscr{F}_{v}$ is a topological isomorphism it suffices to show that it is continuous. The latter conclusion, however, follows from Theorem 4.1 and the explicit construction of $\mathscr{F}_{v}$. This completes the proof of Theorem 4.4 .

Recently, Stout [19] proved Theorem 4.4 for the usual Laplace operator $\Delta$ in $\mathbb{R}^{n}$.

As we have a commutative diagram

$$
\begin{aligned}
& \mathscr{S}_{\Delta}(\bar{U}) \stackrel{i_{2}}{\longrightarrow} H_{\Delta}^{(B)}(U) \stackrel{i_{1}}{\longrightarrow} \mathscr{S}_{\Delta}(U) \\
& \mathscr{\varphi}_{A}(\bar{U}) \stackrel{\cup}{\stackrel{i_{2}}{\longrightarrow}} H_{A}^{(B)}(U) \stackrel{i^{i_{1}}}{\longrightarrow} \mathscr{S}_{A}(U),
\end{aligned}
$$

the pairing $\tilde{h}(\cdot, \cdot)$ induces a continuous mapping

$$
\jmath: \mathscr{Y}_{A}(\bar{U}) \longrightarrow \mathscr{Y}_{A}(U)^{\prime}
$$

which is the restriction of $\mathscr{g}$.

Write $\pi$ for the Szegö projection

$$
\pi: H_{\Delta}^{(B)}(U) \longrightarrow H_{A}^{(B)}(U) .
$$

COROLLARY 4.6. The mapping $\mathfrak{J}$ is a topological isomorphism of the space $\mathscr{Y}_{A}(\bar{U})$ onto $\mathscr{S}_{A}(U)^{\prime}$ if and only if

(1) $\mathscr{S}_{A}(\bar{U})$ is dense in $\mathscr{Y}_{A}(U)$;

(2) $\pi \operatorname{maps} \mathscr{S}_{\Delta}(\bar{U})$ continuously into $\mathscr{Y}_{A}(\bar{U})$.

Proof. According to the general scheme, we have

$$
\Im_{1}=\mathscr{Y}_{A}(U), \quad \Im_{2}=\mathscr{Y}_{A}(\bar{U}), \quad \mathfrak{V}=H_{A}^{(B)}(U),
$$

hence the statement follows from Corollary 2.7. 
Stout [19] proved this theorem for the overdetermined Cauchy-Riemann operator $A=\bar{\partial}$ in $\mathbb{C}^{n}, n>1$.

In [17] it is proved that conditions (1) and (2) of Corollary 4.6 hold for a Dirac operator $A$ in a ball of $X=\mathbb{R}^{n}$ (see Example 3.5).

EXAMPLE 4.7. Let $A$ be a determined elliptic operator with real analytic coefficients on $X$. Then both $A$ and $A^{*}$ possess the unique continuation property in the interior of $X$. Again, condition (1) of Corollary 4.6 is fulfilled.

As in Example 3.6, we consider the extension of $A$ to an operator

$$
T: H_{\Delta}^{(B)}(U) \longrightarrow H_{A^{*}}^{(C)}(U)
$$

where $H_{A^{*}}^{(C)}(U)$ is the closed subspace of the Hardy space $H_{A A^{*}}^{(C)}(U)$ consisting of all the solutions of $A^{*} g=0$ in $U$.

As is proved in Example 3.6, for any $g \in H_{A^{*}}^{(C)}(U)$ we have

$$
\left(A P_{U} n\right)^{-1} g=P_{U}^{1} t\left(\Phi_{U} g\right)
$$

where $P_{U}^{1}$ the Poisson integral of the Dirichlet problem for $A A^{*}$ in $U$.

Let $u \in \mathscr{Y}_{\Delta}(\bar{U})$. Then $A u \in \mathscr{Y}_{A^{*}}(\bar{U})$, and hence it is real analytic in a neighbourhood of $\bar{U}$. By Stokes' formula,

$$
\Phi_{U}(A u)(x)=u(x)+\int_{\partial U} G_{A}\left(K_{\Phi}(x, \cdot), u\right)
$$

for all $x \in U$.

If $u \in \mathscr{Y}_{\Delta}\left(U_{\delta}\right) \cap C^{2 m}\left(\bar{U}_{\delta}, E\right)$ with $\delta>0$ sufficiently small, then by the CauchyKovalevskaya theorem there exist a neighbourhood $O$ of $\partial U$ and a section $v \in C^{m}(\bar{O}, E)$ such that

$$
A v=0 \quad \text { in } O, \quad t(v)=t(u) \text { on } \partial U
$$

By Stokes' formula,

$$
\Phi_{U}(A u)(x)=u(x)-v(x)+\int_{\partial O \cap U} G_{A}\left(K_{\Phi}(x, \cdot), v\right)
$$

for all $x \in O \cap U$.

By the definition of $v$, the right-hand side of the last equality uniquely extends to a solution of $A u=0$ in $O$. It follows that $\Phi_{U}(A u) \in \mathscr{Y}_{\Delta}(O \cup U)$.

Arguing as in the proof of Lemma 4.2, we see that the neighbourhood $O$ does not depend on $u$ but does on $\delta$ and $\partial U$. Moreover, for every solution $u \in \mathscr{Y}_{\Delta}\left(U_{\delta}\right) \cap C^{m}\left(\bar{U}_{\delta}, E\right)$, we get

$$
\|v\|_{C^{m}(\bar{O}, E)} \leq C\|u\|_{C^{m}\left(\bar{U}_{\delta}, E\right)}
$$


with $C>0$ a constant independent of $u$. Hence $\Phi_{U}(A u)$ maps $\mathscr{Y}_{\Delta}(\bar{U})$ continuously into $\mathscr{Y}_{\Delta}(\bar{U})$.

Further, since $X, \partial U, A A^{*}$, and $\left\{B_{j}\right\}$ are real analytic, Theorem A of [10] implies that the Poisson integral $P_{U}^{1} \Phi_{U}(A u)$ is real analytic in a neighbourhood of $\partial U$ provided that $u \in \mathscr{S}_{\Delta}(\bar{U})$. Therefore, $\pi u$ is real analytic in a neighbourhood of $\partial U$, too.

If $\left\{u_{v}\right\}$ converges to $u \in \mathscr{Y}_{\Delta}(\bar{U})$, then $\Phi_{U}\left(A u_{v}\right)$ converges to $\Phi_{U} u$ by (4.32) and $P_{U}^{1} \Phi_{U}\left(A u_{v}\right)$ converges to $P_{U}^{1} \Phi_{U}(A u)$ by [12] (see the proof of Lemma 4.4). Hence $\pi$ maps $\mathscr{Y}_{\Delta}(\bar{U})$ continuously into $\mathscr{S}_{A}(\bar{U})$ (see Section 2.3).

Summarising, we conclude that the mapping $\mathfrak{J}$ is a topological isomorphism of $\mathscr{Y}_{A}(\bar{U})$ onto $\mathscr{Y}_{A}(U)^{\prime}$.

EXAMPLE 4.8. Suppose that $A$ is an overdetermined elliptic differential operator with constant coefficients in $\mathbb{R}^{n}$ as in Example 3.7. If $U \Subset \mathbb{R}^{n}$ is a strictly convex domain with real analytic boundary, then under reasonable assumptions on $A$ the Neumann problem for the tangential complex is solvable at step 1, see Example 3.7 for more details.

Moreover, the Neumann operator $N^{1}$ possesses the analytic hypoellipticity property (cf. [22]). Hence the operator $I-T_{b}^{*} N T_{b}$, that is, the orthogonal projection from $L^{2}\left(\partial U, E_{b}^{0}\right)$ to $\operatorname{ker} T_{b}^{0}$, maps $\mathscr{S}_{\Delta}(\bar{U})$ continuously to $\mathscr{S}_{A}(\bar{U})$. As $L^{2}\left(\partial U, E_{b}^{0}\right)=H_{\Delta}^{(B)}(U)$ and $\operatorname{ker} T_{b}^{0}=H_{A}^{(B)}(U)$, we see that the projection $\pi$ has the same property.

As the domain $U$ is strictly convex, we see that $\mathscr{S}_{A}(\bar{U})$ is dense in $\mathscr{S}_{A}(U)$. By Corollary 3.11, the mapping $\mathfrak{J}$ is a topological isomorphism of $\mathscr{S}_{A}(\bar{U})$ onto $\mathscr{Y}_{A}(U)^{\prime}$. By reflexivity, the transpose $\mathfrak{J}^{\prime}$ gives us a topological isomorphism between the spaces $\mathscr{Y}_{A}(\bar{U})^{\prime}$ and $\mathscr{S}_{A}(U)$.

4.2. Duality in Lebesgue spaces. We now study the case that

$$
\Sigma_{1}=\mathscr{Y}_{\Delta}(U), \quad \Sigma_{2}=\mathscr{Y}_{\Delta}(\bar{U}), \quad V=\mathscr{Y}_{\Delta}^{(0)}(U),
$$

and

$$
i_{1}: \mathscr{Y}_{\Delta}^{(0)}(U) \longrightarrow \mathscr{T}_{\Delta}(U), \quad i_{2}: \mathscr{Y}_{\Delta}(\bar{U}) \longrightarrow \mathscr{Y}_{\Delta}^{(0)}(U)
$$

are natural inclusions.

The mapping $i_{1}$ is always one-to-one and the mapping $i_{2}$ is one-to-one because of the unique continuation property $(U)_{s}$. By a priori elliptic estimates, the mappings $i_{1}$ and $i_{2}$ are continuous. As mentioned, $\mathscr{Y}_{\Delta}^{(0)}(U)$ is a separable Hilbert space with reproducing kernel. To proceed, we thus need to extend the pairing

$$
h(u, v)=\int_{U}(u, v)_{x} d x
$$

from $L^{2}(U, E) \times L^{2}(U, E)$ to $\Sigma_{1} \times \Sigma_{2}$. 
COROLLARY 4.9. Let $\delta>0$ be small enough. Then there are a compact set $K \subset U, \varepsilon_{0}>0$, and $C>0$ depending on $\delta, K$, and $\varepsilon_{0}$ such that for all $u \in \mathscr{Y}_{\Delta}(U)$ and $v \in \mathscr{Y}_{\Delta}\left(U_{\delta}\right) \cap C^{2 m}\left(\bar{U}_{\delta}, E\right)$,

$$
\left|\int_{U_{-\varepsilon}}(u, v)_{x} d x\right| \leq C\|u\|_{C(K, E)}\|v\|_{C^{2 m}\left(\bar{U}_{\delta}, E\right)}
$$

whenever $\varepsilon \in\left(0, \varepsilon_{0}\right]$.

Proof. Let $\varepsilon_{0}$ be the number from Theorem 4.1. Since $\partial U$ is sufficiently smooth, there exists $0<\varepsilon^{\prime} \leq \varepsilon_{0}$ such that for $0<\varepsilon \leq \varepsilon^{\prime}$ we have

$$
\int_{U_{-\varepsilon}}(u, v)_{x} d x=\int_{U_{-\varepsilon^{\prime}}}(u, v)_{x} d x+\int_{\varepsilon}^{\varepsilon^{\prime}} d r \int_{\partial U_{-r}}(u, v)_{x} d s_{-r}
$$

whence

$$
\begin{aligned}
& \left|\int_{U_{-\varepsilon}}(u, v)_{x} d x\right| \\
& \quad \leq \operatorname{meas}(U)\|u\|_{C\left(\bar{U}_{-\varepsilon^{\prime}}, E\right)}\|v\|_{C\left(\bar{U}_{-\varepsilon^{\prime}}, E\right)}+\varepsilon^{\prime} \sup _{r \in\left[\varepsilon, \varepsilon^{\prime}\right]}\left|\int_{\partial U_{-r}}(u, v)_{x} d s_{-r}\right| .
\end{aligned}
$$

Now, the statement of the corollary follows from Theorem 4.1 with $B_{0}=I$ as desired.

In case $\Delta$ is the usual Laplace operator in $\mathbb{R}^{n}$, Zorn [26] proved that the boundary of $U$ should be necessarily real analytic in order that estimate (4.37) may hold.

COROLLARY 4.10. For every solutions $u \in \mathscr{Y}_{\Delta}(U)$ and $v \in \mathscr{Y}_{\Delta}(\bar{U})$, there exists a limit

$$
\tilde{h}(u, v)=\lim _{\varepsilon \rightarrow 0+} \int_{U_{-\varepsilon}}(u, v)_{x} d x
$$

The corresponding pairing $\tilde{h}(\cdot, \cdot)$ is separately continuous on $\mathscr{Y}_{\Delta}(U) \times \mathscr{Y}_{\Delta}(\bar{U})$, and $\tilde{h}(u, v)=h(u, v)$ for all $u \in \mathscr{Y}_{\Delta}^{(0)}(U)$ and $v \in \mathscr{Y}_{\Delta}(\bar{U})$.

Proof. The proof is similar to the proof of Corollary 4.3.

THEOREM 4.11. The mapping $\mathscr{g}: \mathscr{Y}_{\Delta}(\bar{U}) \rightarrow \mathscr{S}_{\Delta}(U)^{\prime}$ induced by (4.40) is a topological isomorphism of these spaces. 
Proof. Since the natural inclusion $i_{2}$ is one-to-one, the mapping $\mathscr{f}$ is oneto-one, too (see Lemma 2.2).

By Corollary 2.5, to prove the surjectivity of the mapping we have to show that the reproducing $\operatorname{kernel} K(\cdot, \cdot)$ of the space $\mathscr{Y}_{\Delta}^{(0)}(U)$ has the following property.

LEMMA 4.12. For every fixed $x \in U$, the Bergman kernel $K(x, \cdot)$ belongs to $E_{x}^{*} \otimes \mathscr{Y}_{\Delta}(\bar{U})$.

Proof. In the proof of Lemma 3.9, we derived the formula

$$
\Delta V(x, \cdot)=-K(x, \cdot)
$$

in $U$ for any fixed $x \in U$ where $V(x, \cdot)$ is a solution of the Dirichlet problem (3.28) for the operator $\Delta^{2}$. Since all the objects $X, \partial U, \Delta$, and $\left\{B_{j}\right\}$ are real analytic, we deduce by [10, Theorem A] that $V(x, \cdot) \in E_{x}^{*} \otimes \mathscr{S}_{\Delta^{2}}(\bar{U})$ for every fixed $x \in U$ (see for instance [12, Lemma 4.4]). Hence the lemma follows as desired.

We have proved that the mapping $\mathscr{F}$ is an isomorphism of vector spaces $\mathscr{Y}_{\Delta}(\bar{U})$ and $\mathscr{Y}_{\Delta}(U)^{\prime}$. The topological arguments are actually the same as those in the proof of Theorem 4.4 .

Since we have a commutative diagram

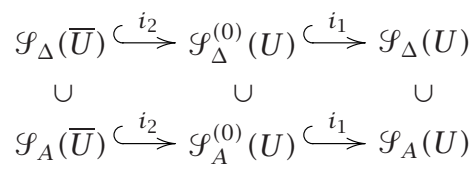

the pairing $\tilde{h}(\cdot, \cdot)$ induces a continuous mapping

$$
\mathfrak{J}: \mathscr{Y}_{A}(\bar{U}) \longrightarrow \mathscr{Y}_{A}(U)^{\prime}
$$

which is the restriction of $\mathscr{F}$.

Write $\pi$ for the Bergman projection

$$
\pi: \mathscr{Y}_{\Delta}^{(0)}(U) \longrightarrow \mathscr{Y}_{A}^{(0)}(U)
$$

COROLLARY 4.13. In order that the mapping $\mathfrak{3}$ be a topological isomorphism of the space $\mathscr{Y}_{A}(\bar{U})$ onto $\mathscr{Y}_{A}(U)^{\prime}$, it is necessary and sufficient that

(1) $\mathscr{S}_{A}(\bar{U})$ be dense in $\mathscr{S}_{A}(U)$;

(2) $\pi$ would map $\mathscr{S}_{\Delta}(\bar{U})$ continuously into $\mathscr{S}_{A}(\bar{U})$. 
Proof. According to the general scheme, we have

$$
\mathfrak{\Im}_{1}=\mathscr{Y}_{A}(U), \quad \Im_{2}=\mathscr{Y}_{A}(\bar{U}), \quad \mathfrak{V}=\mathscr{Y}_{A}^{(0)}(U),
$$

and so the statement follows from Corollary 2.7 .

Zorn [26] proved that the conditions of Corollary 4.13 hold for the CauchyRiemann operator $A=\bar{\partial}$ in any strictly pseudoconvex domain $U$ of $\mathbb{C}^{n}$ with real analytic boundary.

EXAMPLE 4.14. Let $A$ be a determined elliptic operator as in Example 3.12. Since $X, \partial U, A A^{*}$, and $n(\cdot)$ are real analytic, Theorem A of [10] implies that $N A u$ is real analytic in a neighbourhood of $\partial U$ if $u \in \mathscr{Y}_{\Delta}(\bar{U})$. Therefore, $\pi u=$ $u-A^{*} N A u$ is real analytic in a neighbourhood of $\partial U$, too. Furthermore, if $\left\{u_{v}\right\}$ converges to $u \in \mathscr{S}_{\Delta}(\bar{U})$, then $N u_{v}$ converges to $N u$ (cf. the proof of Lemma 4.4 in [12]). Hence $\pi$ maps $\mathscr{Y}_{\Delta}(\bar{U})$ continuously onto $\mathscr{S}_{A}(\bar{U})$. Finally, by the Runge theorem for determined elliptic operators (see [23, Section 4.1.9]), $\mathscr{Y}_{A}(\bar{U})$ is dense in $\mathscr{Y}_{A}(U)$. Hence, according to Corollary 4.13, the mapping $\exists$ is a topological isomorphism of $\mathscr{S}_{A}(\bar{U})$ onto $\mathscr{Y}_{A}(U)^{\prime}$. By reflexivity, the transposed mapping $\mathfrak{J}^{\prime}$ is a topological isomorphism of $\mathscr{Y}_{A}(\bar{U})^{\prime}$ onto $\mathscr{Y}_{A}(U)$.

EXAMPLE 4.15. Assume that $A$ is a column of first-order scalar partial differential operators with constant coefficients in $\mathbb{R}^{n}$. Under familiar assumptions on $A$, the compatibility complex of $A$ is simply a Koszul complex (cf. [22, Section 1.2 .8$]$ ). Let $U \Subset \mathbb{R}^{n}$ be a strictly convex domain with real analytic boundary. Then the Neumann problem for the compatibility complex in $U$ is solvable at step 1, and the Neumann operator $N$ preserves real analytic sections in a neighbourhood of $\bar{U}$. The latter remains valid with convex replaced by pseudoconvex in an appropriate sense (cf. [22, Section 4.1.5]). As the domain $U$ is strictly convex, the subspace $\mathscr{Y}_{A}(\bar{U})$ is dense in $\mathscr{Y}_{A}(D)$. By Corollary 4.13 , the mapping $\mathfrak{J}$ arranges a topological isomorphism of $\mathscr{S}_{A}(\bar{U})$ onto $\mathscr{Y}_{A}(U)^{\prime}$.

EXAMPLE 4.16. Let $X=\mathbb{R}^{n}, n \geq 3$, and let $U \subset \mathbb{R}^{n}$ be a ball and $A$ a Dirac operator in $\mathbb{R}^{n}$ (see Example 3.5). It is proved in [16] that there are systems $\left\{b_{v}\right\}$ and $\left\{c_{\mu}\right\}$ of ( $\mathbb{C}^{k}$-valued) homogeneous harmonic polynomials such that

(a) $\left\{b_{v}\right\}$ is an orthogonal basis in all spaces $\mathscr{P}_{A}^{(0)}\left(U_{\varepsilon}\right), \varepsilon \in \mathbb{R}$, simultaneously;

(b) $\left\{b_{v}\right\} \cup\left\{c_{\mu}\right\}$ is an orthogonal basis in all spaces $\mathscr{Y}_{\Delta}^{(0)}\left(U_{\varepsilon}\right), \varepsilon \in \mathbb{R}$, simultaneously.

Property (a) implies that condition (1) of Corollary 3.11 is satisfied. Moreover, the projection $\pi$ restricted to $\mathscr{S}_{\Delta}^{(0)}\left(U_{\varepsilon}\right), \varepsilon>0$, coincides with the orthogonal projection

$$
\mathscr{Y}_{\Delta}^{(0)}\left(U_{\varepsilon}\right) \longrightarrow \mathscr{Y}_{A}^{(0)}\left(U_{\varepsilon}\right)
$$

It follows that $\pi$ maps $\mathscr{I}_{\Delta}(\bar{U})$ continuously into $\mathscr{S}_{A}(\bar{U})$. Hence Corollary 3.20 holds for $A$. 
4.3. Grothendieck duality. Suppose that $U$ is an open subset of $\stackrel{\circ}{X}$ with $C^{\infty}$ boundary such that $\partial X \cap \bar{U}=\varnothing$ or $\partial X \cap \bar{U}=\partial X$. Set

$$
\Sigma_{1}=\frac{\mathscr{P}_{\Delta}(U)}{\mathscr{H}(X)}, \quad \Sigma_{2}=\frac{\left\{u \in \mathscr{T}_{\Delta}(X \backslash U): t(u)=0 \text { on } \partial X\right\}}{\mathscr{H}(X)},
$$

and

$$
V=\mathscr{Y}_{\Delta}^{(m)}(X \backslash \bar{U}, \partial X) \ominus \mathscr{H}(X)
$$

" $\ominus$ " meaning the orthogonal complement with respect to the scalar product $h_{X \backslash \bar{U}}(\cdot, \cdot)$.

For each $[u] \in \Sigma_{2}$, we set

$$
i_{2}[v]=p(v)
$$

where $v$ is a representative of the class $[v]$ and $p(v)$ the orthogonal projection of $v$ to $V$ in $\mathscr{S}_{\Delta}^{(m)}(X \backslash \bar{U}, \partial X)$. If $v_{1}, v_{2} \in[v]$, then $v_{1}-v_{2} \in \mathscr{H}(X)$ whence $p\left(v_{1}-v_{2}\right)=0$. It follows that the mapping $i_{2}: \Sigma_{2} \rightarrow V$ is well defined and continuous as is easy to check.

Further, for $u \in V$, we set

$$
i_{1} u=\left[\mathscr{E}_{X \backslash \bar{U}}(u)\right]
$$

that is, the equivalence class in $\Sigma_{1}$ corresponding to the restriction of $\mathscr{E}_{X \backslash \bar{U}}(u)$ to $U$.

We are now in a position to extend the sesquilinear pairing $h_{X \backslash \bar{U}}(\cdot, \cdot)$ from $V \times V$ to $\Sigma_{1} \times \Sigma_{2}$. Namely, if $[v] \in \Sigma_{2}$, then there exists a domain $O \Subset U$ with smooth boundary $\partial O$ such that $v \in \mathscr{T}_{\Delta}(X \backslash O)$ for all $v \in[v]$. Given any $[u] \in$ $\Sigma_{1}$ and $[v] \in \Sigma_{2}$, we set

$$
\tilde{h}_{X \backslash \bar{U}}([u],[v])=-\int_{\partial O} G_{\Delta}\left(*_{E} v, u\right),
$$

where $u \in[u]$ and $v \in[v]$.

LEMMA 4.17. As defined by (4.51), the pairing $\tilde{h}_{X \backslash \bar{U}}(\cdot, \cdot)$ does not depend on the choice of $O$ and $u \in[u], v \in[v]$. Moreover, it is separately continuous, and

$$
\tilde{h}_{X \backslash \bar{U}}\left(i_{1} u,[v]\right)=h_{X \backslash \bar{U}}\left(u, i_{2}[v]\right)
$$

for all $u \in V$ and $[v] \in \Sigma_{2}$.

Proof. By Stokes' formula, we get

$$
\int_{\partial O} G_{\Delta}\left(*_{E} v, u\right)=\int_{O}(\Delta u, v)_{x} d x-\int_{O}(u, \Delta v)_{x} d x=0
$$


for all $v \in \mathscr{H}(X)$. Similarly,

$$
-\int_{\partial O} G_{\Delta}\left(*_{E} v, u\right)=\int_{X \backslash O}(\Delta u, v)_{x} d x-\int_{X \backslash O}(u, \Delta v)_{x} d x=0
$$

for all $u \in \mathscr{H}(X)$. This means that $\tilde{h}_{X \backslash \bar{U}}(\cdot, \cdot)$ does not depend on the choice of $u \in[u]$ and $v \in[v]$.

Let $O^{\prime} \Subset U$ be another domain with smooth boundary such that $\Delta v=0$ near $X \backslash O^{\prime}$ for all $v \in[v]$. Without loss of generality, we can assume that $O \Subset O^{\prime}$. Then by Stokes' formula, we get

$$
\int_{\partial O^{\prime}} G_{\Delta}\left(*_{E} v, u\right)-\int_{\partial O} G_{\Delta}\left(*_{E} v, u\right)=\int_{\partial\left(O^{\prime} \backslash O\right)} G_{\Delta}\left(*_{E} v, u\right)=0
$$

for both $u$ and $v$ belong to $\mathscr{Y}_{\Delta}\left(\bar{O}^{\prime} \backslash O\right)$. Thus, $\tilde{h}_{X \backslash \bar{U}}(\cdot, \cdot)$ is independent of the particular choice of $O$.

Obviously, $\tilde{h}_{X \backslash \bar{U}}(\cdot, \cdot)$ is separately continuous if the spaces $\Sigma_{1}$ and $\Sigma_{2}$ are endowed with canonical quotient topology.

Since any solution $u \in V$ has finite order of growth near $\partial U$, it follows from (4.55) that

$$
\begin{aligned}
\tilde{h}_{X \backslash \bar{U}}\left(i_{1} u,[v]\right) & =-\int_{\partial O} G_{\Delta}\left(*_{E} v, \mathscr{E}_{X \backslash \bar{U}} u\right) \\
& =-\int_{\partial U} G_{\Delta}\left(*_{E} v, \mathscr{E}_{X \backslash \bar{U}} u\right) \\
& =-\int_{\partial U}\left(\left(t\left(\mathscr{E}_{X \backslash \bar{U}} u\right), n(A v)\right)_{x}-\left(n\left(A \mathscr{E}_{X \backslash \bar{U}} u\right), t(v)\right)_{x}\right) d s \\
& =\int_{\partial U}\left(\left(n\left(A \mathscr{E}_{X \backslash \bar{U}} u\right), t\left(\mathscr{E}_{X \backslash \bar{U}} v\right)\right)_{x}-(t(u), n(A v))_{x}\right) d s \\
& \left.\left.=h_{X \backslash \bar{U}}(u, v)-\int_{X}\left(H^{\mathscr{E}} X \bar{U}\right), H^{\mathscr{E}} X \backslash \bar{U}\right)\right)_{x} d x \\
& =h_{X \backslash \bar{U}}(u, v),
\end{aligned}
$$

the last equality being a consequence of the fact that $u \perp \mathscr{H}(X)$ with respect to $h_{X \backslash \bar{U}}(\cdot, \cdot)$.

Finally, we obtain

$$
h_{X \backslash \bar{U}}(u, v)=h_{X \backslash \bar{U}}(p(u), v)=h_{X \backslash \bar{U}}(u, p(v))=h_{X \backslash \bar{U}}\left(u, i_{2}[v]\right) \text {, }
$$

which shows the lemma.

We thus conclude that there is a mapping $\mathscr{f}: \Sigma_{2} \rightarrow \Sigma_{1}^{\prime}$ induced by the pairing $\tilde{h}_{X \backslash \bar{U}}(\cdot, \cdot)$. 
THEOREM 4.18. The mapping

$$
\mathscr{F}: \frac{\left\{u \in \mathscr{T}_{\Delta}(X \backslash U): t(u)=0 \text { on } \partial X\right\}}{\mathscr{H}(X)} \longrightarrow\left(\frac{\mathscr{P}_{\Delta}(U)}{\mathscr{H}(X)}\right)^{\prime}
$$

induced by (4.51) is a topological isomorphism of these spaces.

Proof. As $p(u)=0$ implies $u \in \mathscr{H}(X)$, we see that $i_{2}$ is one-to-one. Then $\mathscr{f}$ is one-to-one, too (see Lemma 2.2).

We prove the surjectivity of $\mathscr{f}$. To this end, we pick a continuous linear functional $\mathscr{F}$ on $\Sigma_{1}$. Then $\mathscr{F}$ can be thought of as a functional on $\mathscr{S}_{\Delta}(U)$ vanishing on $\mathscr{H}(X)$.

Since $\mathscr{Y}_{\Delta}(U)$ is a subspace of $C_{\text {loc }}(U, E)$, the space of continuous sections of $E$ over $U$, this functional can be extended, by the Hahn-Banach theorem, to an $E^{*}$-valued measure $m$ with compact support in $U$ orthogonal to $\mathscr{H}(X)$.

Take a domain $O^{\prime} \Subset U$ containing the support of $m$. Then, for every $u \in$ $\mathscr{Y}_{\Delta}(U)$ and $x \in O^{\prime}$, we have

$$
u(x)=-\int_{\partial O^{\prime}} G_{\Delta}\left(K_{G}(x, \cdot), u\right)+H\left(x_{O^{\prime}} u\right)(x),
$$

where $G$ is the Hodge parametrix for the Dirichlet problem in $X$ (see Section 2.4).

Hence

$$
\langle\mathscr{F},[u]\rangle=-\int_{\partial O^{\prime}} G_{\Delta}\left(*_{E} v, u\right)
$$

with an element $u \in[u]$, and

$$
v(y)=*_{E}^{-1}\left\langle d m, K_{G}(\cdot, y)\right\rangle=G\left(*_{E}^{-1} d m\right)(y)
$$

for $y$ away from the support of $m$.

Since $G$ is a Hodge parametrix, we see that $t(v)=0$ on $\partial X$. Moreover, we get

$$
\Delta v=*_{E}^{-1} d m-H\left(*_{E}^{-1} \mathscr{F}\right)=*_{E}^{-1} d m,
$$

for $\mathscr{F}$ vanishes on $\mathscr{H}(X)$. It follows that $\Delta v=0$ in a neighbourhood of $X \backslash O^{\prime}$, that is, $v$ determines an equivalence class $[v]$ in $\Sigma_{2}$. Obviously, we have $\mathscr{E}[v]=$ $\mathscr{F}_{\mathcal{F}}$, which proves the surjectivity of $\mathscr{F}$.

Finally, the topological arguments are actually the same as those in Theorem 4.4 .

4.4. Duality in Sobolev spaces. From now on, we assume that the manifold $X$, the bundles $E, F$, and the coefficients of the operator $A$ are real analytic. 
Let $U \Subset X$ be a domain with real analytic boundary. We introduce the spaces of the solutions

$$
\Sigma_{1}=\frac{\mathscr{P}_{\Delta}(U)}{\mathscr{H}(X)}, \quad \Sigma_{2}=\frac{\mathscr{P}_{\Delta}(\bar{U})}{\mathscr{H}(X)}, \quad V=\mathscr{Y}_{\Delta}^{(m)}(U) \ominus \mathscr{H}(X),
$$

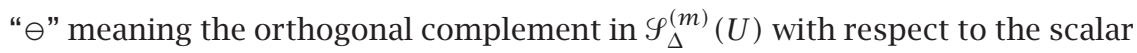
product $h_{U}(\cdot, \cdot)$. We endow $V$ with the scalar product $h_{U}(\cdot, \cdot)$, thus making it a Hilbert space.

For $[u] \in \Sigma_{2}$, we set

$$
E_{U}[v]=\left[\mathscr{E}_{U}(u)\right]
$$

with $u$ a representative of $[u]$. We check that this definition does not depend on the particular choice of $u \in[u]$. Indeed, it was proved in [12] (see the proof of Corollary 4.1) that the mapping

$$
\mathscr{E}_{U}: \mathscr{S}_{\Delta}(\bar{U}) \longrightarrow\left\{u \in \mathscr{Y}_{\Delta}(X \backslash U): t(u)=0 \text { on } \partial X\right\}
$$

is a topological isomorphism of the spaces. Therefore, we conclude that $\mathscr{E}_{U}(u)$ belongs to $\mathscr{Y}_{\Delta}(X \backslash U)$ and satisfies $t\left(\mathscr{E}_{U}(u)\right)=0$ on $\partial X$. In particular, if $u \in$ $\mathscr{H}(X)$, then $\mathscr{E}_{U}(u)=u$, and this gives us the independence on the choice of $u \in[u]$ as desired.

As the space $\mathscr{H}(X)$ is finite dimensional, we immediately obtain the following lemma.

\section{LEMMA 4.19. The mapping}

$$
E_{U}: \frac{\mathscr{P}_{\Delta}(\bar{U})}{\mathscr{H}(X)} \rightarrow \frac{\left\{u \in \mathscr{T}_{\Delta}(X \backslash U): t(u)=0 \text { on } \partial X\right\}}{\mathscr{H}(X)}
$$

is a topological isomorphism of the spaces.

Of course, Lemma 4.19 and Theorem 4.18 already imply that the spaces $\Sigma_{2}$ and $\Sigma_{1}^{\prime}$ are topologically isomorphic. However, we want to derive an explicit construction of this duality.

To this end, we set

$$
i_{1}: V \longrightarrow \Sigma_{1}, \quad i_{2}: \Sigma_{2} \longrightarrow V
$$

to be the natural inclusions. They are obviously one-to-one and continuous.

We define an extension $\tilde{h}_{U}(\cdot, \cdot)$ of $h_{U}(\cdot, \cdot)$ as follows:

$$
\tilde{h}_{U}([u],[v])=\tilde{h}_{X \backslash \bar{U}}\left([u], E_{U}[v]\right)
$$

(see (3.62)). 
LEMmA 4.20. As defined by (4.68), the pairing $\tilde{h}_{U}(\cdot, \cdot)$ does not depend on the choice of $u \in[u]$ and $v \in[v]$. Moreover, it is separately continuous and satisfies

$$
\tilde{h}_{U}\left(i_{1} u,[v]\right)=h_{U}\left(u, i_{2}[v]\right)
$$

for all $u \in V$ and $[v] \in \Sigma_{2}$.

Proof. The pairing is independent of the choice of $u \in[u]$ and $v \in[v]$ because so are the pairing $\tilde{h}_{X \backslash \bar{U}}$ and the mapping $E_{U}$.

Moreover, from the definition of $\mathscr{E}_{U}$, it follows that $\mathscr{E}_{X \backslash \bar{U}} \mathscr{E}_{U}=1$ on $\mathscr{Y}_{\Delta}^{(m)}(U)$. Hence, by Lemma 4.17 we get

$$
\begin{aligned}
\tilde{h}_{U}\left(i_{1} u,[v]\right) & =\tilde{h}_{X \backslash \bar{U}}\left(i_{1} \mathscr{E}_{X \backslash \bar{U}} \mathscr{E}_{U}(u), E_{U}([v])\right) \\
& =h_{X \backslash \bar{U}}\left(\mathscr{E}_{X \backslash \bar{U}} \mathscr{E}_{U}(u), i_{2} E_{U}([v])\right) \\
& =h_{U}\left(u, i_{2}[v]\right)
\end{aligned}
$$

as desired.

THEOREM 4.21. The mapping

$$
\mathscr{F}: \frac{\mathscr{P}_{\Delta}(\bar{U})}{\mathscr{H}(X)} \rightarrow\left(\frac{\mathscr{\varphi}_{\Delta}(U)}{\mathscr{H}(X)}\right)^{\prime}
$$

induced by (4.68) is a topological isomorphism of these spaces.

Proof. This follows from Lemma 4.19 and Theorem 4.18.

As we have a commutative diagram

$$
\begin{aligned}
& \frac{\mathscr{P}_{\Delta}(\bar{U})}{\mathscr{H}(X)} \stackrel{i_{2}}{\longrightarrow} \mathscr{\varphi}_{\Delta}^{(m)}(U) \ominus \mathscr{H}(X) \stackrel{i_{1}}{\longrightarrow} \frac{\mathscr{P}_{\Delta}(U)}{\mathscr{H}(X)} \\
& \cup \\
& \frac{\mathscr{P}_{A}(\bar{U})}{\mathscr{H}(X)} \stackrel{i_{2}}{\longrightarrow} \mathscr{Y}_{A}^{(m)}(U) \ominus \mathscr{H}(X) \stackrel{i_{1}}{\longrightarrow} \frac{\mathscr{P}_{A}(U)}{\mathscr{H}(X)},
\end{aligned}
$$

the pairing $\tilde{h}_{U}(\cdot, \cdot)$ induces a continuous mapping

$$
\mathfrak{J}: \frac{\mathscr{I}_{A}(\bar{U})}{\mathscr{H}(X)} \longrightarrow\left(\frac{\mathscr{P}_{A}(U)}{\mathscr{H}(X)}\right)^{\prime}
$$

which is the restriction of $\mathscr{F}$.

Denote by $\pi$ the orthogonal projection

$$
\pi: \mathscr{Y}_{\Delta}^{(m)}(U) \ominus \mathscr{H}(X) \longrightarrow \mathscr{Y}_{A}^{(m)}(U) \ominus \mathscr{H}(X) .
$$


COROLLARY 4.22. The mapping $\mathfrak{J}$ is a topological isomorphism of spaces (4.73) if and only if

(1) $\mathscr{S}_{A}(\bar{U})$ is dense in $\mathscr{Y}_{A}(U)$;

(2) $\pi$ maps $\mathscr{Y}_{\Delta}(\bar{U})$ continuously into $\mathscr{S}_{A}(\bar{U})$.

Proof. According to the general scheme, we have

$$
\mathfrak{\Im}_{1}=\frac{\mathscr{T}_{A}(U)}{\mathscr{H}(X)}, \quad \mathfrak{\Im}_{2}=\frac{\mathscr{S}_{A}(\bar{U})}{\mathscr{H}(X)}, \quad \mathfrak{v}=\mathscr{Y}_{A}^{(m)}(U) \ominus \mathscr{H}(X),
$$

hence the statement follows from Corollary 2.7.

EXAMPLE 4.23. Assume that $A$ is a homogeneous determined elliptic operator with constant coefficients of order $m$ in $X=\mathbb{R}^{n}$ with $n>2 m$ (see Example 3.12). As we have already seen in the latter example, the projection $\pi$ is given by

$$
(M u)(x)=-\int_{\partial U} G_{A}\left(K_{\Phi}(x-\cdot), u\right)
$$

for any $u \in H^{m}(U, E)$, where $\Phi$ is a fundamental solution of convolution type for $A$.

If $u \in \mathscr{Y}_{\Delta}\left(U_{\varepsilon}\right) \cap C^{m}\left(\bar{U}_{\varepsilon}, E\right)$ with $\varepsilon>0$ small enough, then by the CauchyKovalevskaya theorem there exist a neighbourhood $O$ of $\partial U$ and a section $v \in C^{m}(\bar{O}, E)$, such that

$$
A v=0 \quad \text { in } O, \quad t(v)=t(u) \text { on } \partial U .
$$

By the definition of $M$, we get $M u=M v$ in $U$. Then Stokes' formula yields

$$
M u(x)=v(x)-\int_{(\partial O) \cap U} G_{A}\left(K_{\Phi}(x-\cdot), v\right)
$$

for all $x \in O \cap U$. The right-hand side of the latter equality uniquely extends to a solution of $A u=0$ in $O$. Hence we deduce that $M u \in \mathscr{Y}_{A}(U \cup O)$.

Arguing as in the proof of Lemma 4.2, we see that $O$ is actually independent of $u$, but it depends on $\varepsilon$ and $\partial U$. Moreover, for every $u \in \mathscr{Y}_{\Delta}\left(U_{\varepsilon}\right) \cap C^{m}\left(\bar{U}_{\varepsilon}, E\right)$, we have

$$
\|v\|_{C^{m}(\bar{O}, E)} \leq C\|u\|_{C^{m}\left(\bar{U}_{\varepsilon}, E\right)}
$$

with $C>0$ a constant independent of $u$. Hence $M$ maps $\mathscr{I}_{\Delta}(\bar{U})$ continuously into $\mathscr{Y}_{A}(\bar{U})$.

EXAMPLE 4.24. Let $A$ be a determined elliptic operator as in Example 3.22. Then the condition (1) of Corollary 4.22 is fulfilled. Assume for simplicity that $\mathscr{H}(X)=0$. 
As we have seen in Example 3.22, $\pi=I-T_{U}\left(A T_{U}\right)^{-1} A$ where the operator $\left(A T_{U}\right)^{-1}$ is given by (3.74).

It is well known that the kernel of $\Phi_{A^{*}}$ is real analytic outside the diagonal $\{x=y\}$. Recall that $\Phi_{A^{*}}$ stands for a two-sided fundamental solution of the operator $A^{*}$ near $\bar{U}$. Hence it follows that the boundary integral in (3.74) is real analytic in a neighbourhood $O$ of $\bar{U}$.

We thus conclude that $\left(A T_{U}\right)^{-1} f$ is real analytic in $O$ if $f$ has the same property.

If $u \in \mathscr{S}_{\Delta}(\bar{U}) \cap C^{2 m}\left(\bar{U}_{\varepsilon}, E\right)$ with sufficiently small $\varepsilon>0$ such that $U_{\varepsilon} \Subset O$, then $\left(A T_{U}\right)^{-1} A u$ is real analytic in a neighbourhood of $U_{\varepsilon}$.

We see that $T_{U} f$ is real analytic in a neighbourhood of $\bar{U}$ if $f$ is real analytic in $U_{\varepsilon}$.

By the Cauchy-Kovalevskaya theorem, there exist a neighbourhood $O^{\prime}$ of $\partial U$ and a section $v \in C^{m}\left(\bar{O}^{\prime}, E\right)$ such that

$$
A v=f \quad \text { in } O^{\prime}, \quad t(v)=0 \quad \text { on } \partial U .
$$

Then Stokes' formula yields

$$
T_{U} f(x)=T_{U \backslash O^{\prime}} f(x)+v(x)-\int_{\left(\partial O^{\prime}\right) \cap U} G_{A}\left(K_{G A^{*}}(x, \cdot), v\right)
$$

for all $x \in O^{\prime} \cap U$.

By the definition of $v$, the right-hand side of the last equality uniquely extends to $O^{\prime}$ as a real analytic section. Therefore, the same conclusion is valid for $T_{U} f$.

Finally, arguing as in the proof of Lemma 4.2, we obtain that the neighbourhood $O^{\prime}$ does not depend on $f$ but does on $\varepsilon$ and $\partial U$. Moreover, for every $f \in C^{m}\left(\bar{U}_{\varepsilon}, E\right)$ real analytic in $U_{\varepsilon}$, we get

$$
\|v\|_{C^{m}\left(\bar{O}^{\prime}, E\right)} \leq C\|f\|_{C^{m}\left(\bar{U}_{\varepsilon}, F\right)}
$$

with $C>0$ a constant independent of $f$. Hence, the continuity of $\pi$ follows from the continuity of the operators $A, T_{U}, H, \Phi_{A^{*}}$, and formula (3.74).

EXAMPLE 4.25. Let $X=\mathbb{R}^{n}, n \geq 3, U$ be a ball in $\mathbb{R}^{n}$, and $A$ a Dirac operator in $\mathbb{R}^{n}$ (see Example 3.5). In [16] it is proved that there are systems $\left\{\boldsymbol{b}_{v}\right\}$ and $\left\{c_{\mu}\right\}$ of homogeneous harmonic polynomials with values in $\mathbb{C}^{k}$ such that

(a) $\left\{b_{v}\right\}$ is an orthogonal basis in all spaces $\mathscr{Y}_{A}^{(1)}\left(U_{\varepsilon}\right)$, where $\varepsilon \in \mathbb{R}$ simultaneously;

(b) $\left\{b_{v}\right\} \cup\left\{c_{\mu}\right\}$ is an orthogonal basis in all spaces $\mathscr{Y}_{\Delta}^{(1)}\left(U_{\varepsilon}\right), \varepsilon \in \mathbb{R}$, simultaneously. 
Property (a) implies that condition (1) of Corollary 4.22 is satisfied. Moreover, the projection $\pi$ restricted to $\mathscr{S}_{\Delta}^{(1)}\left(U_{\varepsilon}\right), \varepsilon>0$, coincides with the orthogonal projection

$$
\mathscr{Y}_{\Delta}^{(1)}\left(U_{\varepsilon}\right) \longrightarrow \mathscr{Y}_{A}^{(1)}\left(U_{\varepsilon}\right)
$$

It follows that $\pi$ maps $\mathscr{S}_{\Delta}(\bar{U})$ continuously into $\mathscr{S}_{A}(\bar{U})$. Hence Corollary 4.22 is valid for $A$.

ACKNOWLedgment. A. Shlapunov was partially supported by the University of Potsdam and Regional Science Fund of Krasnoyarsk.

\section{REFERENCES}

[1] N. Aronszajn, Theory of reproducing kernels, Trans. Amer. Math. Soc. 68 (1950), 337-404.

[2] L. A. Aĭzenberg, The general form of a continuous linear functional on the space of functions holomorphic in a convex region of $C^{n}$, Dokl. Akad. Nauk SSSR 166 (1966), no. 5, 1015-1018 (Russian).

[3] S. R. Bell, A duality theorem for harmonic functions, Michigan Math. J. 29 (1982), no. 1, 123-128.

[4] S. R. Bell and H. P. Boas, Regularity of the Bergman projection and duality of holomorphic function spaces, Math. Ann. 267 (1984), no. 4, 473-478.

[5] A. A. Dezin, General Questions of the Theory of Boundary Value Problems, Nauka, Moscow, 1980 (Russian).

[6] V. P. Havin, Spaces of analytic functions, Math. Analysis, 1964, Akad. Nauk SSSR Inst. Naučn. Informacii, Moscow, 1966, pp. 76-164 (Russian).

[7] L. Hörmander, The Analysis of Linear Partial Differential Operators. I, Grundlehren der Mathematischen Wissenschaften, vol. 256, SpringerVerlag, Berlin, 1983.

[8] A. Martineau, Sur les fonctionnnelles analytiques et la transformation de FourierBorel, J. Analyse Math. 11 (1963), 1-164 (French).

[9] M. Morimoto, An Introduction to Sato's Hyperfunctions, Translations of Mathematical Monographs, vol. 129, American Mathematical Society, Rhode Island, 1993.

[10] C. B. Morrey Jr. and L. Nirenberg, On the analyticity of the solutions of linear elliptic systems of partial differential equations, Comm. Pure Appl. Math. 10 (1957), 271-290.

[11] M. Nacinovich and A. A. Shlapunov, On iterations of the Green integrals and their applications to elliptic differential complexes, Math. Nachr. 180 (1996), 243284.

[12] M. Nacinovich, A. A. Shlapunov, and N. N. Tarkhanov, Duality in the spaces of solutions of elliptic systems, Ann. Scuola Norm. Sup. Pisa Cl. Sci. (4) 26 (1998), no. 2, 207-232.

[13] Ya. Roitberg, Elliptic Boundary Value Problems in the Spaces of Distributions, Mathematics and Its Applications, vol. 384, Kluwer Academic Publishers, Dordrecht, 1996.

[14] M. Schechter, Negative norms and boundary problems, Ann. of Math. (2) 72 (1960), no. 3, 581-593.

[15] B.-W. Schulze, A. A. Shlapunov, and N. N. Tarkhanov, Green Integrals on Manifolds with Cracks, preprint 2000/12, 2000. 
[16] A. A. Shlapunov, Spectral decomposition of Green's integrals and existence of $W^{s, 2}$-solutions of matrix factorizations of the Laplace operator in a ball, Rend. Sem. Mat. Univ. Padova 96 (1996), 237-256.

[17] _ On a dual space for solutions of a Dirac operator in ball, Proc. Conf. Symmetry in Natural Science, Inst. of Comp. Mod., Krasnoyarsk, 2000, pp. 262265.

[18] A. A. Shlapunov and N. N. Tarkhanov, Bases with double orthogonality in the Cauchy problem for systems with injective symbols, Proc. London Math. Soc. (3) 71 (1995), no. 1, 1-52.

[19] E. L. Stout, Harmonic duality, hyperfunctions and removable singularities, Izv. Ross. Akad. Nauk Ser. Mat. 59 (1995), no. 6, 133-170.

[20] E. J. Straube, Harmonic and analytic functions admitting a distribution boundary value, Ann. Scuola Norm. Sup. Pisa Cl. Sci. (4) 11 (1984), no. 4, 559-591.

[21] N. N. Tarkhanov, The Cauchy Problem for Solutions of Elliptic Equations, Mathematical Topics, vol. 7, Akademie Verlag, Berlin, 1995.

[22] - Complexes of Differential Operators, Mathematics and Its Applications, vol. 340, Kluwer Academic Publishers, Dordrecht, 1995.

[23] _ The Analysis of Solutions of Elliptic Equations, Mathematics and Its Applications, vol. 406, Kluwer Academic Publishers, Dordrecht, 1997.

[24] K. Yosida, Functional Analysis, Grundlehren der Mathematischen Wissenschaften, vol. 123, Springer-Verlag, Berlin, 1965.

[25] S. V. Znamenskiĭ, A geometric criterion of strong linear convexity, Funktsional. Anal. i Prilozhen. 13 (1979), no. 3, 83-84.

[26] P. Zorn, Analytic functionals and Bergman spaces, Ann. Scuola Norm. Sup. Pisa Cl. Sci. (4) 9 (1982), no. 3, 365-404.

A. Shlapunov: Krasnoyarsk State University, pr. Svobodnyi 79, 660041 Krasnoyarsk, Russia

E-mail address: sh1apuno@1an.krasu.ru

N. Tarkhanov: Universität Potsdam, Institut für Mathematik, Postfach 6015 53, 14415 Potsdam, Germany

E-mail address: tarkhanov@math. uni-potsdam.de 


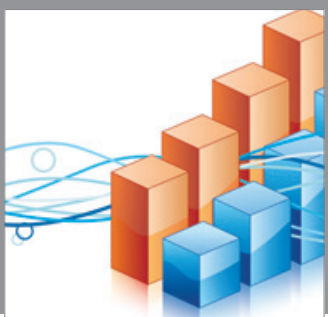

Advances in

Operations Research

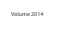

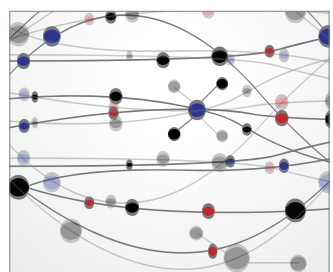

\section{The Scientific} World Journal
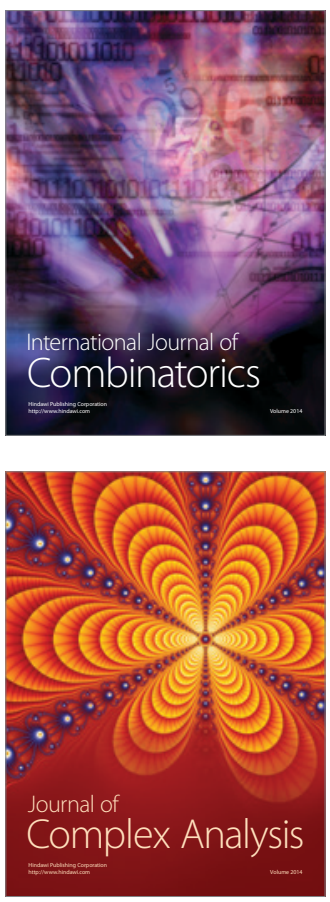

International Journal of

Mathematics and

Mathematical

Sciences
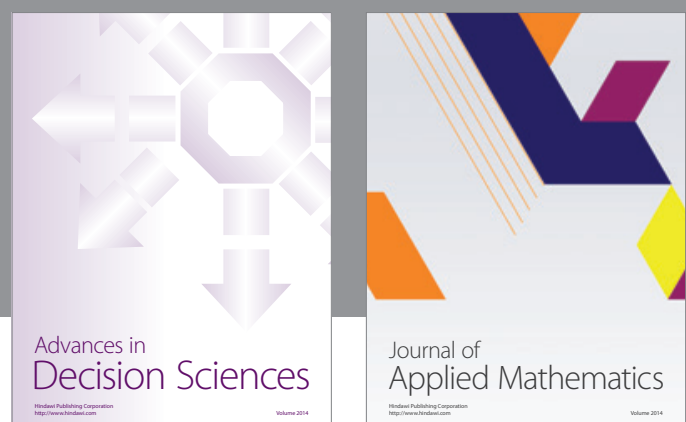

Journal of

Applied Mathematics
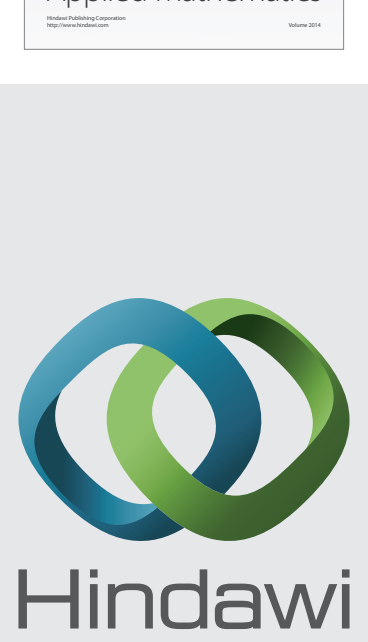

Submit your manuscripts at http://www.hindawi.com
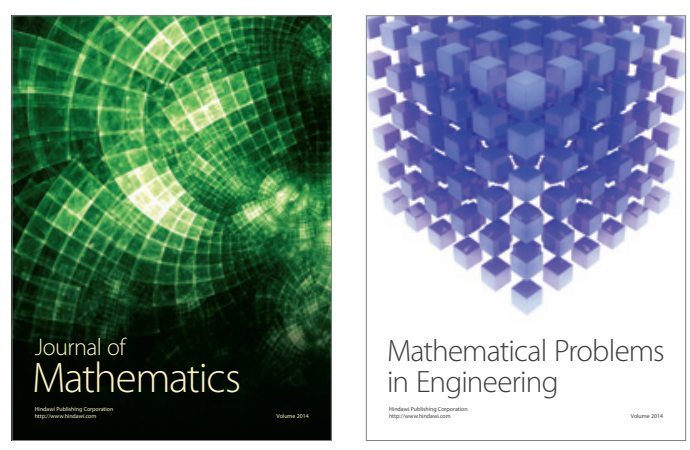

Mathematical Problems in Engineering
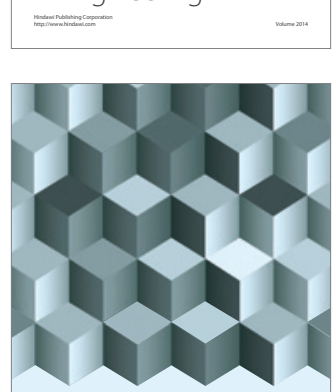

Journal of

Function Spaces
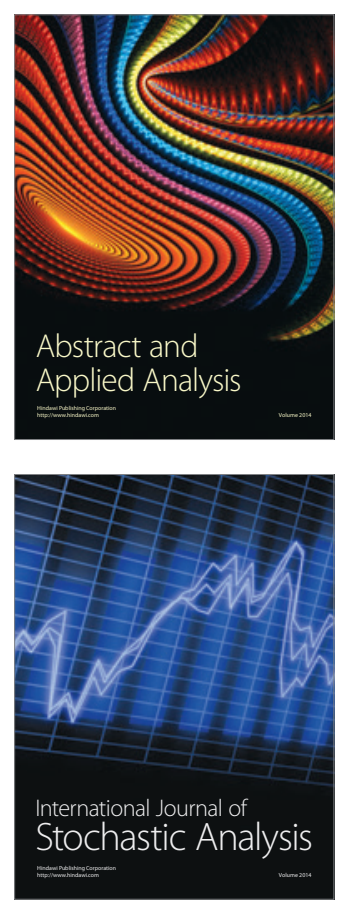

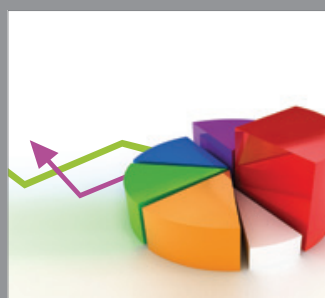

ournal of

Probability and Statistics

Promensencen
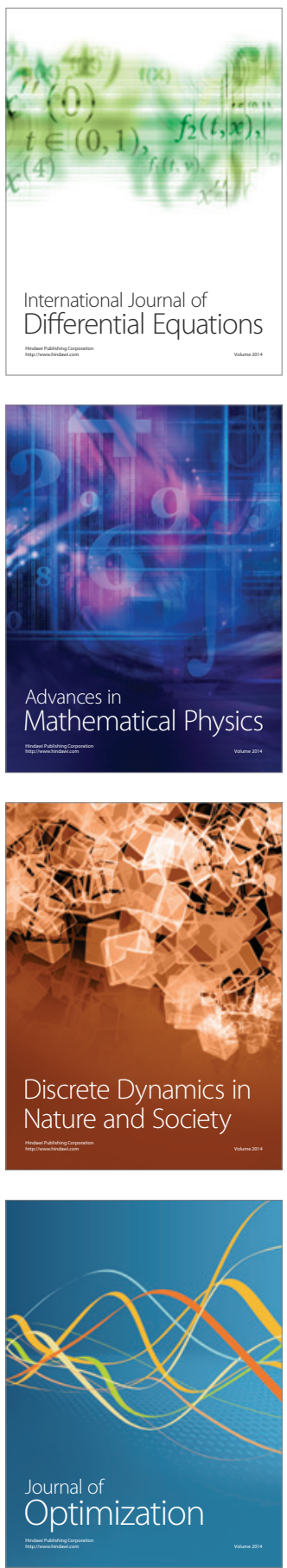